\title{
Brain differences associated with personality traits: a structural and resting-state fMRI approach
}

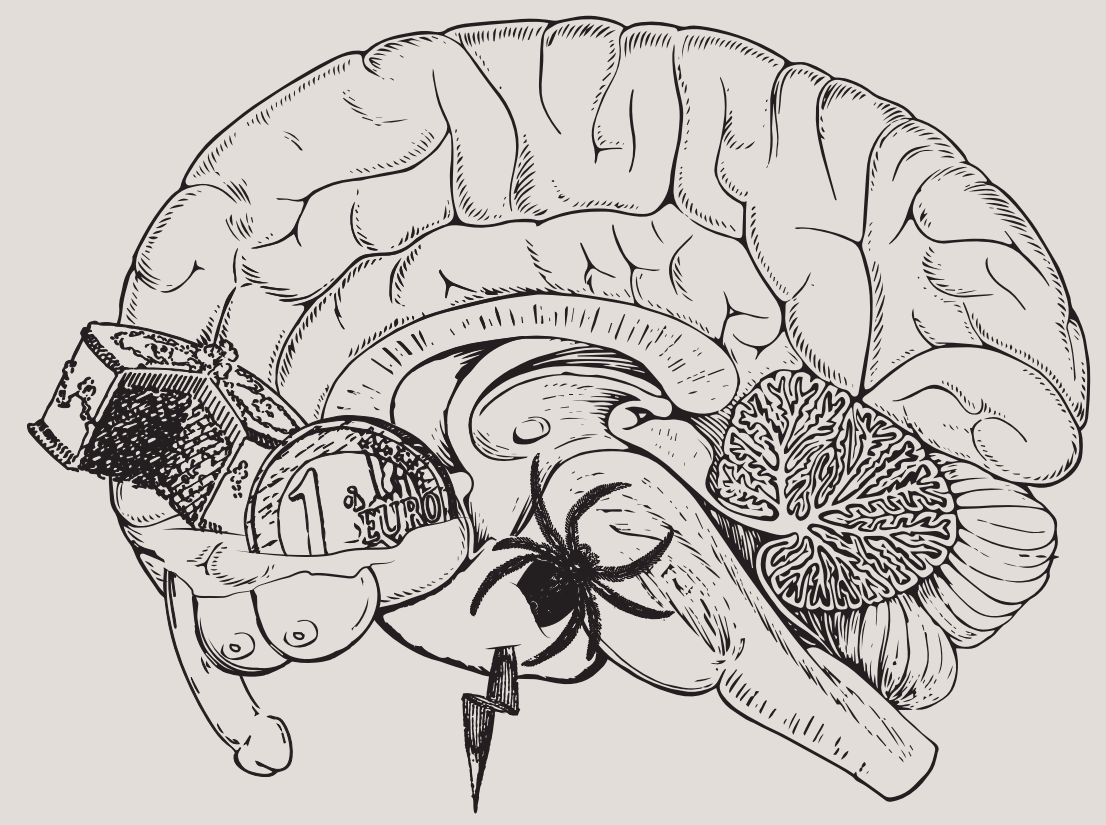

Jesús Adrián Ventura

Supervisors:

César Ávila Rivera

Víctor Costumero Ramos 



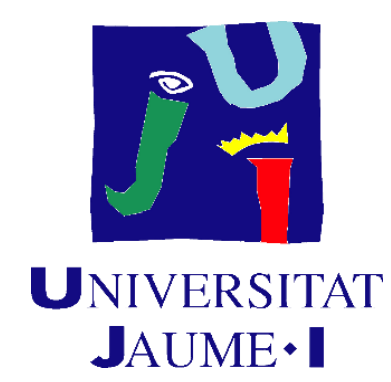

\section{Programa de Doctorado en Psicología}

Escuela de Doctorado de la Universitat Jaume I

\section{Brain differences associated with personality traits: a structural and resting-state fMRI approach}

Memoria presentada por Jesús Adrián Ventura para optar al grado de doctor/a por la Universitat Jaume I

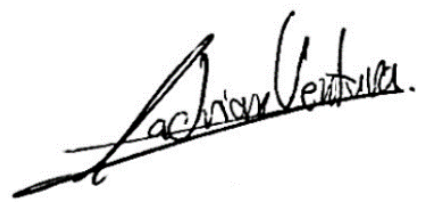

Jesús Adrián Ventura

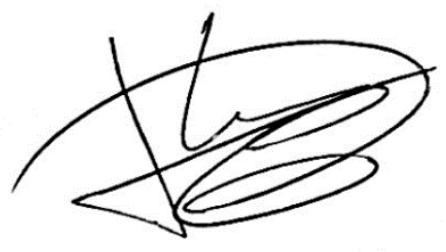

César Ávila Rivera

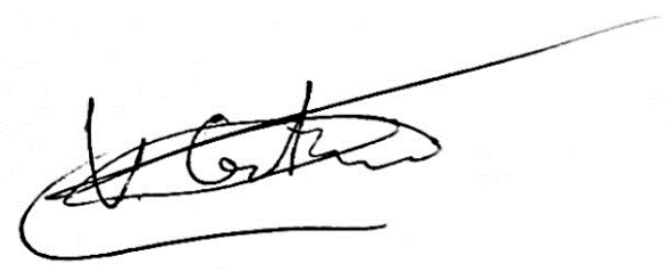

Víctor Costumero Ramos 



\section{Financiación recibida}

Esta tesis doctoral ha sido posible gracias a la financiación recibida por parte del Ministerio de Educación, Cultura y Deporte [Ayudas para la formación de profesorado universitario (FPU15/00825)] y por el Ministerio de Ciencia, Innovación y Universidades [Ayudas de movilidad para estancias breves (EST18/00677)].

Adicionalmente, también se ha contado con la financiación de los proyectos de investigación AICO/2018/038 (Generalitat Valenciana) y UJI-B2018-22 (Universitat Jaume I). 

Tesis por compendio de las siguientes publicaciones:

- Linking personality and brain anatomy: a structural MRI approach to Reinforcement Sensitivity Theory

Autores: Jesús Adrián-Ventura*, Víctor Costumero, Maria Antònia Parcet, César Ávila

Revista: Social Cognitive and Affective Neuroscience

DOI: doi.org/10.1093/scan/nsz011

Factor de impacto: 3.66 (2018)

- Reward network connectivity "at rest" is associated with reward sensitivity in healthy adults: A resting-state fMRI study

Autores: Jesús Adrián-Ventura*, Víctor Costumero, Maria Antònia Parcet, César Ávila

Revista: Cognitive, Affective, \& Behavioral Neuroscience

DOI: doi.org/10.3758/s13415-019-00688-1

Factor de impacto: 2.66 (2018)

- The manifestation of individual differences in sensitivity to punishment during resting state is modulated by eye state

Autores: Víctor Costumero*, Jesús Adrián-Ventura*, Elisenda Bueichekú, Anna MiróPadilla, María-Ángeles Palomar-García, Lidón Marin-Marin, Esteban Villar-Rodríguez, Naiara Aguirre, Alfonso Barrós-Loscertales, César Ávila

Sometido (en revisión)

Esta tesis dispone de la aceptación de los coautores de las publicaciones que el doctorando/a presenta como tesis y su renuncia expresa a presentarlas como parte de otra tesis doctoral. 



\section{Imagen de portada y contraportada}

La imagen de la portada y contraportada de esta tesis ha sido creada por la diseñadora Raquel Adrián Ventura (@dissenyavant). Durante el proceso de diseño se han utilizado los programas MRIcroGL $\odot$, Adobe Illustrator ${ }^{\circledR}$, Adobe Photoshop® y Adobe InDesing ${ }^{\circledR}$.

¡Gracias Raquel! 



\section{Agradecimientos / Acknowledgements}

Realizar una tesis doctoral no es un camino fácil, pero es más llevadero si se cuenta con la ayuda oportuna. Por ello, en las siguientes líneas me gustaría agradecer el apoyo recibido durante estos últimos años a muchas personas que considero importantes tanto en mi vida personal como profesional, ya que sin ellos esta tesis no hubiera sido posible.

En primer lugar, me gustaría agradecer a mis directores de tesis, el Dr. César Ávila y el Dr. Víctor Costumero, por darme la oportunidad de llevar a cabo este trabajo. César, gracias por acogerme en el grupo, abrirme la mente al mundo de la neuroimagen y por tu guía y valiosos consejos durante estos años. Víctor, gracias por haberme ayudado en tantas cosas, tu punto de vista y experiencia ha aportado un plus de calidad enorme a la tesis.

En segundo lugar, a mis compañeros de grupo, y en especial a Eli y Anna. Eli, gracias por tu amistad y tus consejos. Tu dedicación y trabajo es un ejemplo a seguir y también una motivación extra para seguir creciendo como investigador. Anna, ha sido un placer-y una suerte - trabajar contigo. Todos los buenos ratos que hemos echado tomando café han sido vitales para poder seguir adelante y olvidar los malos ratos que se pasan a veces en la ciencia. Gracias por todo. Al resto de mis compañeros, presentes y pasados: Marian, Maya, Noe, Patri, Paola, Javi, Mireia, Naiara, Esteban, Lidón, Lara, Helena, Alfonso, Cris, Carla... he trabajado muy a gusto con vosotros, todos contribuís a que nuestro grupo haya sido, y siga siendo, un sitio agradable y enriquecedor. También me gustaría hacer una mención especial a los últimos en llegar: Esteban, Lidón, Lara y Helena. Ha sido genial compartir este tiempo con vosotros, habéis demostrado desde el primer día una gran dedicación y compañerismo. Además, hacéis que el día a día sea más ameno y entretenido.

En tercer lugar, no quiero olvidarme de la Dra. Marie Banich. Thank you, Marie, for giving me the opportunity to work with you and your fantastic group. I had a good time in Boulder. Also, during my stay I was able to learn new neuroimaging techniques that will surely be useful in my future career as a researcher.

Por último, el agradecimiento a mi familia. A mis padres, por mostrarme su apoyo y sentirse orgullosos de todos mis logros. Y cómo no, a mi hermana. Raquel, eres genial. Pese a que somos de mundos distintos (Psicología e Ingeniería), no puedo sino sentir una gran admiración por todo lo que haces, tienes un talento que inspira.

A todos, ;muchas gracias! 



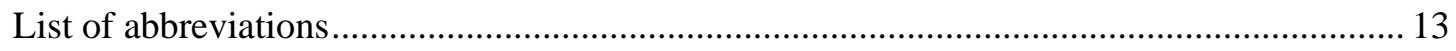

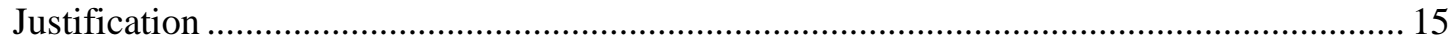

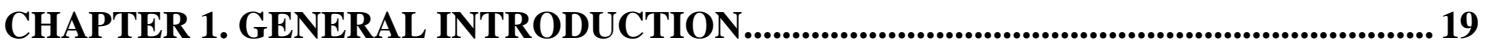

1. The foundations of Reinforcement Sensitivity Theory ........................................................ 19

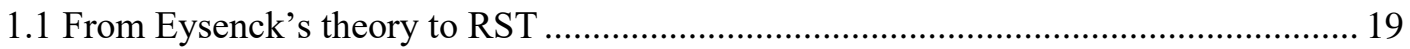

2. The revised Reinforcement Sensitivity Theory ……........................................................... 22

2.1 The Behavioural Approach System (BAS) …….......................................................... 22

2.2 The Fight/Flight/Freeze System (FFFS).................................................................... 24

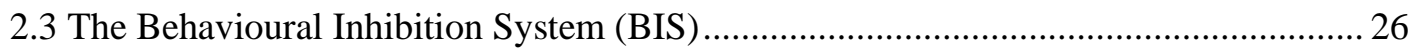

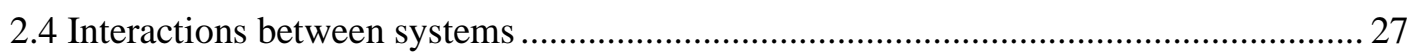

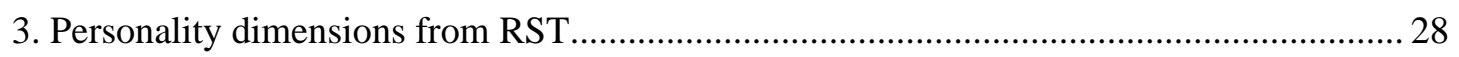

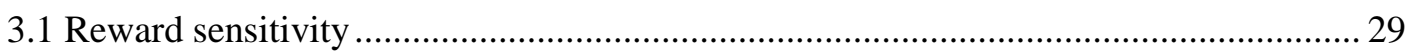

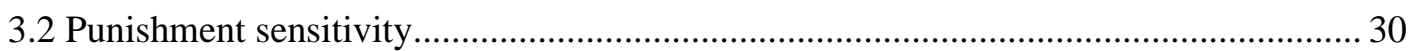

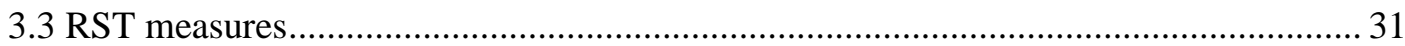

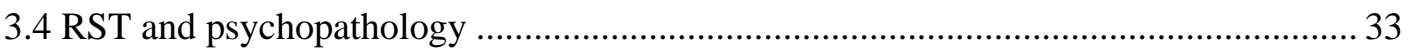

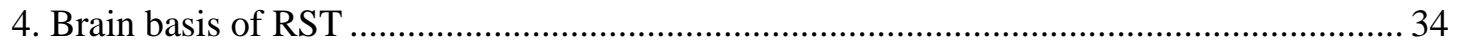

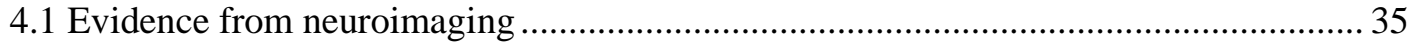

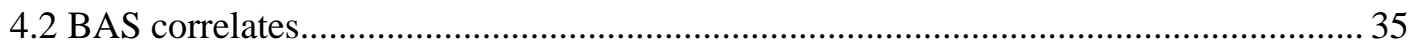

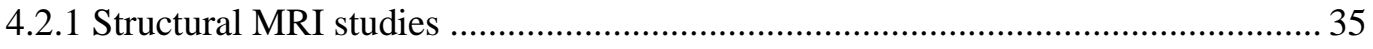

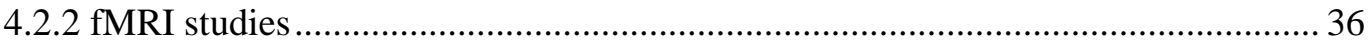

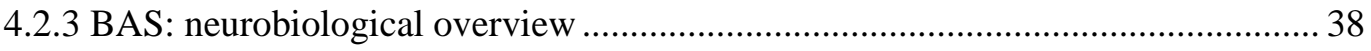

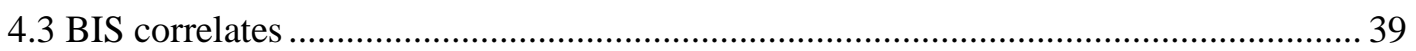

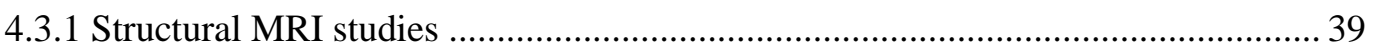

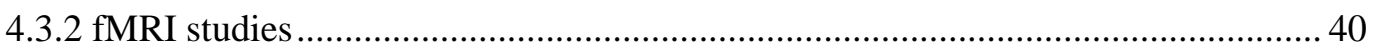

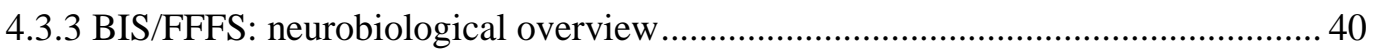

CHAPTER 2. EXPERIMENTAL SECTION_........................................................................ 43

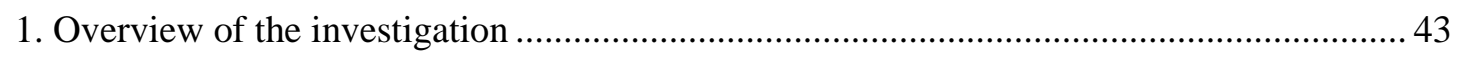

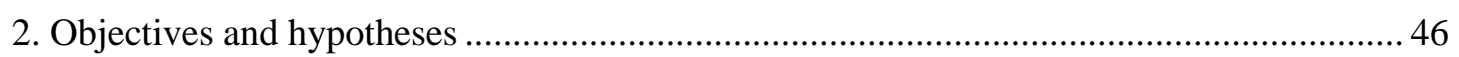

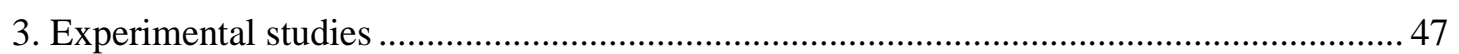

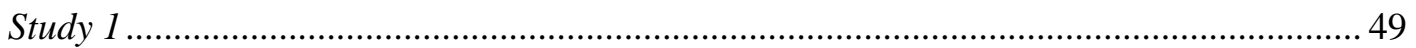

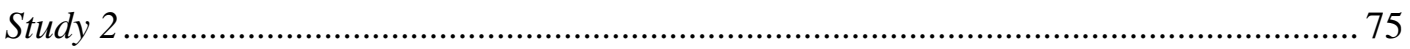

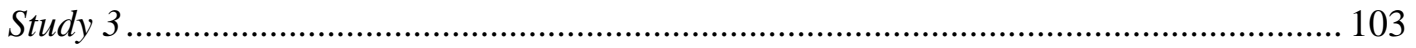

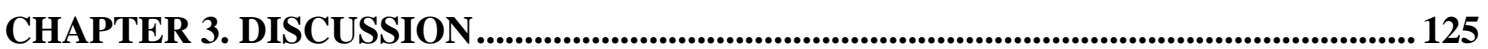

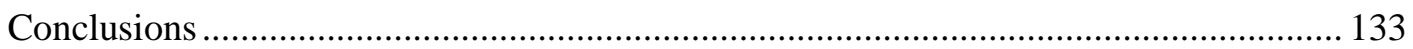

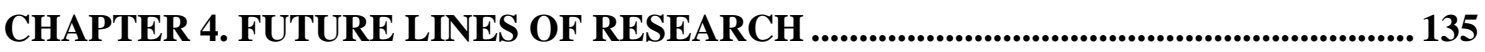

References for general introduction and discussion....................................................... 137 



\section{List of abbreviations}

ACC: anterior cingulate cortex

BAS: behavioural approach/activation system

BIS: behavioural inhibition system

BOLD: blood-oxygen-level-dependent

DTI: diffusion tensor imaging

EO/EC: eyes open/eyes closed

FDR: false discovery rate

FFFS: fight/flight/freeze system

fMRI: functional magnetic resonance imaging

FWE: family-wise error

GMV: grey matter volume

HA: harm avoidance

mPFC: medial prefrontal cortex

MRI: magnetic resonance imaging

NAcc: nucleus accumbens

NS: novelty seeking

OFC: orbitofrontal cortex

PAG: periaqueductal grey

ROI: region of interest

rsFC: resting-state functional connectivity

RST: reinforcement sensitivity theory

SP: sensitivity to punishment

SPM: statistical parametric mapping

SR: sensitivity to reward

SPSRQ: sensitivity to punishment and sensitivity to reward questionnaire

TCI: temperament and character inventory

VBM: voxel-based morphometry

vmPFC: ventromedial prefrontal cortex

VTA: ventral tegmental area 


\section{Justification}

Personality characterises one's habitual behaviour. This "person's nature" comes from stable, well-defined individual differences in personality traits that form the core of specific behavioural patterns. As such, personality traits guide our daily actions. For this reason, studying individual differences in personality can help us to understand normal and abnormal behaviours. Therefore, by analysing this relationship we can better understand why people exhibit very different behaviours in similar circumstances. To illustrate this idea, think about the following example:

«Imagine that two people are on a diet. Both are truly committed to the same aim: losing weight. For a month, they have been doing exercise and eating healthy food (also drastically reducing the number of calories). Consequently, they have lost weight, which makes them happy and fulfilled, even though some days have been very tough. To celebrate this achievement, they are having a party with some friends. However, one of the guests is bringing a delicious chocolate cake. Subsequently, both of them start to think about the cake and the possibility of going off their diet just for one day, given that they really want to try it. After rethinking and evaluating the pros and cons, one of them finally decides to eat the cake, but the other does not». Interestingly, the behavioural outcomes of this "to eat or not to eat" dilemma could have been predicted based on individual differences in personality, given that they reflect stable behavioural patterns over time. For example, if we observe that a person is a) highly sensitive to rewards, we could expect a higher probability of impulsive reward-guided behaviours, whereas if a person is b) highly sensitive to punishments, we could expect a lower probability of these behaviours. So, if we consider that the two people from our example show traits a) and b), respectively, can we predict whether they will eat cake? The answer is yes because the a) trait promotes the detection of rewarding stimuli and reward-oriented behaviours (i.e. trying the cake) without paying attention to negative consequences (i.e. going off their diet). This example shows how personality traits lead to individual differences in the person's decision-making process. We can identify, then, a number of cognitive domains that take part in this process (i.e. attention, motivation, learning, executive functions, etc.) and are modulated by personality.

Personality models, however, have classically been developed by means of factorial analysis in order to establish the minimum explanatory personality dimensions and their biological basis. Thus, in the 1960s and 70s, some comprehensive theories emerged, mainly Eysenck's Introversion-Extraversion Theory and Gray's Reinforcement Sensitivity Theory (RST), as psychobiological frameworks to study individual behavioural differences based on personality traits. These models used a factor analytic approach to describe main personality traits and then gave biological explanations for these factors. Surprisingly, this second component 
disappeared with the boom of cognitive neuroscience in the 1990s. Whereas factorial analysis was undoubtedly valuable for the development of these personality models, new evidence arising from cognitive neuroscience suggested that, at the biological level, personality dimensions may be captured by higher-order cognitive domains, thus hindering the isolation of specific biological traits. Hence, personality models have been adapted and updated to include a cognitive perspective, which in turn provides more accurate predictions in terms of behaviour. In this line, behavioural research carried out in recent decades has been prominent in the study of individual differences in personality and their relationship with cognitive processes. In addition, some investigations have also been dedicated to linking these differences to neurobiological features. This growing body of literature at both behavioural and biological levels led scientists to develop and remodel different theories based on brain-behaviour interactions. One of the most influential has been RST, originally developed by Jeffrey Gray in the 1970s. Since then, RST has been updated and refined, thus generating a comprehensive framework with which to study behavioural patterns in both healthy and clinical populations based on the main personality dimensions depicted in the model: Sensitivity to Punishment (SP) and Sensitivity to Reward (SR).

However, it is important to highlight that, even though the neuropsychological description of RST is quite exhaustive, its foundations were based on animal findings, and less research has been dedicated to testing their correspondence in humans. For this reason, further studies are needed in order to extend and adapt this model to human cognition. By doing so, we would be able to confirm the relationship between personality traits (e.g. SP and SR) and their neurobiological basis in humans. Providing a better description of the brain areas involved in the reward and punishment systems would be of great interest in the study of the neuroscience of personality, given that both systems are fundamental in a wide variety of behaviours.

Currently, fortunately, non-invasive Magnetic Resonance Imaging (MRI) techniques offer a fantastic set of toolboxes designed to analyse the brain structure and function and their relationship with behavioural measures (both task-based and self-reported questionnaires). Thus, MRI has emerged as a promising method to advance in this research field. Nonetheless, due to the recent development of these techniques, little research has been carried out so far. In fact, only a few studies have reported brain patterns linked to the personality traits depicted in RST. Moreover, some of these studies were conducted with small samples or with outdated methods. Therefore, we believe that new investigations with state-of-the-art methods and larger samples would provide new and valuable evidence about RST. This would be useful for establishing a very detailed and thorough framework to analyse and understand human behaviour based on individual differences in personality. In other words, we would be able to understand what cognitive processes are modulated by personality traits and their influence on behavioural outcomes. For instance, returning to the aforementioned example, do individuals with high $S R$ 
show lower behavioural inhibition and then try the cake? Or, are individuals with high SP more concerned about the negative consequences of going off their diet? Likewise, could we predict the development of clinical disorders (e.g. anxiety, depression, addiction, or psychopathy) based on personality? And more importantly: Can we link these traits to a biological substratum in humans?

Therefore, the aim of this thesis is to provide new evidence to support and expand the classical neuropsychological framework of RST in humans. This would be of great interest in the research field of personality psychology, given that we could better characterise normal and pathological behaviours and their biological roots based on personality traits. 


\section{CHAPTER 1. GENERAL INTRODUCTION}

\section{The foundations of Reinforcement Sensitivity Theory}

Reinforcement Sensitivity Theory (RST), originally developed by Jeffrey A. Gray, has been one of the most influential models of personality in the past 50 years. On the basis of Hans J. Eysenck's introversion-extraversion theory (H. J. Eysenck, 1967; H. J. Eysenck \& Eysenck, 1985), Gray (1970) developed an alternative psychophysiological theory of personality that expanded and adapted Eysenck's theory based on emerging psychophysiological evidence. Therefore, RST offered a new perspective on the psychology of personality that provided a robust framework that helped scientists to better understand the psychological traits associated with particular behaviours and their biological correlates.

Although RST was further developed and updated in subsequent decades, it is interesting to understand the origin and foundations of this model. To this end, it is necessary to make a distinction between Eysenck's and Gray's models of personality. In the next section, we offer an overview of Eysenck's introversion-extroversion theory and the alternative hypothesis contemplated by Gray.

\subsection{From Eysenck's theory to RST}

Eysenck's theory of introversion-extroversion was one of the first attempts to conceptualize and describe the psychophysiological basis of personality. This theory (H. J. Eysenck, 1960, 1967) postulated the existence of two different dimensions: introversionextraversion and neuroticism-emotional stability. The first, introversion-extraversion, was associated with the ascending reticular activating system (ARAS; also known as the "reticulocortical" circuit), whereas the neuroticism dimension was related to the limbic system (the "reticulo-limbic" circuit). Within Eysenck's theory, the ARAS was intended to control the cortical arousal elicited by incoming neutral stimuli, whereas the limbic system was activated by emotional stimuli (see Matthews \& Gilliland, 1999).

Importantly, individual differences in the response of these biological systems (i.e. in the level of arousability/activation) were thought to reflect differences at the behavioural level, that is, differences in personality traits (Gray, 1987a; Matthews \& Gilliland, 1999). Indeed, the dimensions of extraversion and neuroticism were originally developed to account for different psychiatric conditions, such as hysteria/psychopathy and those derived from anxiety states (H. J. 
Eysenck, 1957, 1967; H. J. Eysenck \& Claridge, 1962; Gray, 1970). Behaviourally, introvert individuals show a lower ARAS activation threshold in comparison with extraverts, thus resulting in higher arousal levels under low intensity stimulation (i.e. under low stimulation, introverts reach high arousal levels, whereas extroverts need more stimulation to reach those levels). Moreover, cortical arousal in both introverts and extraverts can be modulated by transmarginal inhibition (TMI), a brain mechanism involved in reducing excessive - and disturbing - arousal levels (Corr, 2004; Matthews \& Gilliland, 1999; Pickering \& Corr, 2008). In fact, there is a direct link between the arousal level and cognitive performance (e.g. on attentional tasks). Hence, intermediate arousal levels would predict better performance, thus generating an inverted-U relationship between these variables. Then, individuals are motivated to seek an "optimal" arousal level based on their degree of arousability (i.e. introverts would look for low stimulant contexts, whereas extraverts would favour high stimulant environments). In addition to the introversionextraversion dimension, Eysenck proposed the neuroticism trait as a predisposing factor for psychiatric disorders (H. J. Eysenck, 1957; H. J. Eysenck \& Claridge, 1962; Ormel et al., 2013). This trait was thought to reflect individual differences in the activation of the limbic system, thus indicating the degree of emotionality (i.e. reactions to emotional stimuli), and so neuroticism manifests the emotional stability-instability of an individual. Consequently, high neuroticism individuals show a pattern of emotional instability with a negative cognitive bias (see Ormel et al., 2013 for a review).

Later, Eysenck included a third factor, psychoticism, as a personality dimension within his model (H. J. Eysenck, 1992, 1997; H. J. Eysenck \& Eysenck, 1976). This trait was originally developed to explain the common personality factor across psychotic disorders (e.g. schizophrenia/schizotypy, psychopathy, or bipolar disorders). Subsequently, however, psychoticism was established as the core of antisocial spectrum behaviours (i.e. lack of conformity, impulsivity, hostility, aggression, etc.), thus linking these "asocial" features to personality aspects (Corr, 2010; Vollema \& van den Bosch, 1995). Likewise, this diminished behavioural inhibition was biologically linked to the dopaminergic system.

In combination, individual differences in the activity of the aforementioned psychobiological systems underlie the variability in personality traits (e.g. extraversion, neuroticism, and psychoticism), which, in extreme cases, can lead to abnormal behaviours and the development of psychiatric disorders (H. J. Eysenck, 1957; H. J. Eysenck \& Claridge, 1962; Gray, 1970). This is of particular interest for the research field of personality psychology and psychopathology, given that this early assumption was pivotal in considering personality traits as psychopathological predisposition/vulnerability factors in the non-clinical population. 
However, although Eysenck's theory provided a solid framework for studying personality and its relationship with psychopathological traits, some theoretical and physiological pitfalls contributed to a paradigm shift. These points are summarised in Corr (2004). Thus, RST emerged as a new model designed to resolve some of Eysenck's theory limitations. To this aim, Gray first applied a $30^{\circ}$ rotation over Eysenck's original introversion-extraversion and neuroticism-stability axes (see Figure 1; Gray, 1970). This rotation led to the distinction of two new, more causally efficient dimensions of personality: Sensitivity to Punishment (SP, reflecting anxiety) and Sensitivity to Reward (SR, reflecting impulsivity). Subsequently, Gray proposed SP and SR as the neuropsychological basis for the Behavioural Inhibition System (BIS) and the Behavioural Approach System (BAS), respectively (Pickering, Corr, \& Gray, 1999).

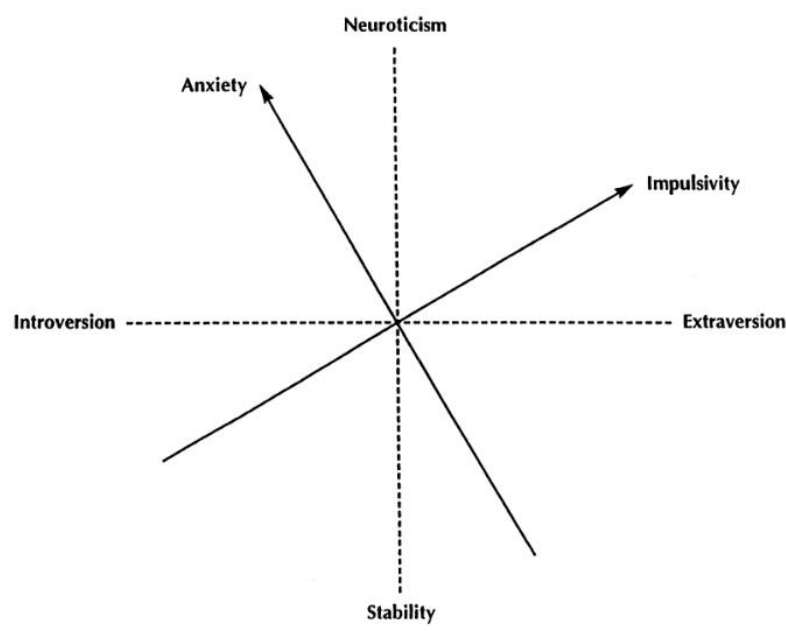

Figure 1. Correspondence between Eysenck's personality traits and Gray's anxiety and impulsivity dimensions depicted in RST. Extracted from Pickering et al. (1999).

Based on this original conception, Gray elaborated a well-defined taxonomy of personality (Gray, 1981, 1982, 1987a, 1987b; Pickering et al., 1999). On the one hand, the BIS was described as the neuropsychological system responsible for resolving conflicts (principally in approach-avoidance situations), acting as "comparator" in the presence of negative conditioned stimuli (i.e. signals of punishment or frustrative non-reward). At the neurobiological level, the septo-hippocampal formation was defined as the core region of this system. On the other hand, the BAS was responsible for promoting approach behaviours towards positive conditioned stimuli (i.e. signals of reward or non-punishment). This system was thought to be activated by dopaminergic inputs from the mesolimbic system to striatal and frontal areas. Finally, years later, Gray (1987b, 1987c, 1995) introduced a third system, the Fight/Flight System (FFS), which activates in the presence of unconditioned aversive stimuli. Behavioural reactions emerging from 
this system (i.e. avoidance of threat) would be driven by the amygdala, the hypothalamus, and the periaqueductal grey (PAG).

Thus, RST established a comprehensive framework that provided information about the neurobiological systems responsible for taking control of behaviour when facing emotional stimuli. Furthermore, RST also emerged as a solid model to study psychopathological conditions associated with Gray's anxiety and impulsivity dimensions and the reactivity of the BIS, BAS, and FFS. In the next section, we discuss the changes in RST in subsequent years, which led to the revised $\mathrm{RST}$.

\section{The revised Reinforcement Sensitivity Theory}

Since the publication of RST in 1982, a growing body of literature, mainly from psychopharmacology experimentation in animal models, has provided new findings that complement and expand the existing foundations of RST. This revision of the model concluded with the publication of the second edition of RST (the revised RST, rRST; Gray \& McNaughton, 2000). In the following sections, we will summarise the main issues introduced in the rRST in relation to the BIS, BAS, and FFS. For an exhaustive comparison of the original RST and the rRST, comprehensive reviews are available elsewhere (Corr, 2004; McNaughton \& Corr, 2004, 2008).

\subsection{The Behavioural Approach System (BAS)}

The rRST proposed the BAS as the brain system involved in detecting and promoting responses to appetitive conditioned and unconditioned rewarding stimuli (positive valence) or the absence of punishment. Behaviourally, the BAS mediates reactions to these stimuli through two different mechanisms: motivation and learning (Pickering \& Gray, 2001; Pickering \& Smillie, 2008). The former refers to the increase in arousal levels that engage and redirect ongoing behaviours towards appetitive stimuli, whereas the latter is responsible for the stimulus-stimulus and stimulus-response association, that is, the reinforcing effect of a given stimulus. In addition, the BAS has been related to positive affect and the emotional state of "hope" in relation to rewarding stimuli and "relief" in relation to avoidance of aversive stimuli (Fowles, 2002).

At the biological level, the BAS has traditionally been linked to the dopaminergic brain system (i.e. the so-called cortico-basal ganglia circuit). This system comprises a well-described 
circuitry originating in the midbrain [e.g. the substantia nigra $(\mathrm{SN})$ pars compacta and ventral tegmental area (VTA)] that projects to striatal [e.g. the dorsal (caudate nucleus and putamen) and ventral (nucleus accumbens (NAcc) and ventral pallidum) striatum] and frontal areas. These structures and connections are anatomically organized in different tracts, which have classically been defined as the main "dopaminergic pathways": the mesocortical, mesolimbic, and nigrostriatal tracts (Arias-Carrión, Stamelou, Murillo-Rodríguez, Menéndez-González, \& Pöppel, 2010; Björklund \& Dunnett, 2007; Cools, 2008; Haber, 2014; Hayes, Duncan, Xu, \& Northoff, 2014; Wise, 2004). These pathways (for a visual representation, see Figure 2) have been profusely studied due to their involvement in a number of cognitive, affective, and motivational processes. The nigrostriatal pathway (i.e. the SN-dorsal striatum connection) has mainly been associated with motor activity (Bourdy et al., 2014; Haber \& Knutson, 2010; Wise, 2004), although some investigations have also shown its influence on learning (habit-formation) and motivational processes (Wise, 2009). On the other hand, both the mesocortical pathway (i.e. the connection between the VTA and medial prefrontal structures) and the mesolimbic pathway (i.e. the VTA-ventral striatum connection) have traditionally been involved in goal-directed behaviours. In fact, these systems are involved in the detection and evaluation of novel stimuli, thus coding the hedonic value of appetitive reinforcers (the so-called "liking"). Then, stimulus value-coding is matched with specific behaviours via instrumental reinforcement ("learning"), which in turn endorses incentive-motivated approach behaviours ("wanting") toward alert signals that predict these stimuli. Hence, through the different dopaminergic cortico-basal ganglia loops, approach behaviours are gradually reinforced (Berridge, Robinson, \& Aldridge, 2009; BrombergMartin, Matsumoto, \& Hikosaka, 2010; Bunzeck \& Düzel, 2006; Cools, 2008; Hayes et al., 2014; Krebs, Heipertz, Schuetze, \& Duzel, 2011; Montague, Hyman, \& Cohen, 2004; Richter \& Gruber, 2018; Sesack \& Grace, 2010; Wise, 2004).

Together, the mesocortical and mesolimbic pathways, also referred to as the mesocorticolimbic system (Hayes et al., 2014; Wise, 2004), represent the biological structure of the BAS (Depue \& Collins, 1999; Pickering \& Gray, 1999, 2001). Thus, BAS activity reflects individual differences in the functioning of this system, which in turn involves different behaviours when approaching rewarding stimuli. 


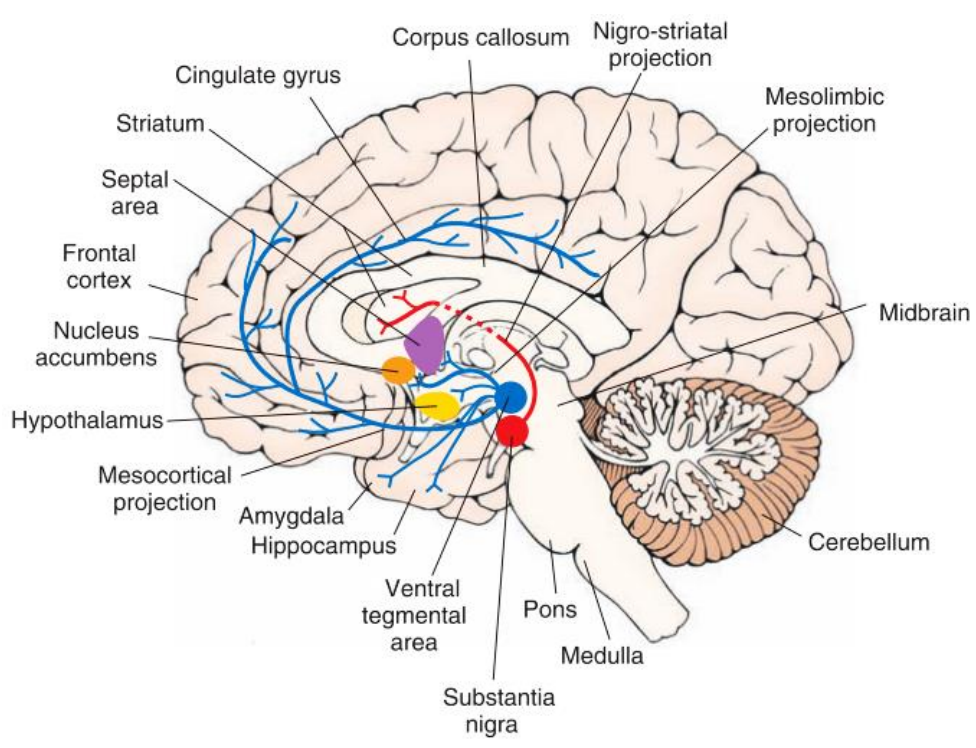

Figure 2. Overview of the main areas and tracts involved in the dopaminergic reward system. Extracted from Siegel \& Sapru (2010).

\subsection{The Fight/Flight/Freeze System (FFFS)}

The rRST proposed the FFFS as the brain system involved in responding to conditioned and unconditioned aversive stimuli (Corr, 2004; Gray \& McNaughton, 2000). In addition to fight and flight, the rRST included a new behavioural component, freezing, associated with the fear dimension and avoidance behaviours. Together, these three components conformed the FFFS, the neurobehavioural system intended to keep the organism away from threatening, harmful stimuli (McNaughton \& Corr, 2004).

Behaviours arising from the FFFS foster active avoidance behaviours in the presence of aversive stimuli. These behaviours underlie the construct of defensive avoidance, whereby the organism escapes from harmful situations by promoting fight, flight, or freezing actions. In turn, this construct depends on the defensive direction. This dimension establishes two different defensive categories that take control of behaviour with the aim of averting dangerous stimuli. Thus, as Blanchard \& Blanchard (1990b, 1990a) stated, an organism could emit defensive avoidance behaviours (activated via the FFFS) or defensive approach behaviours (via the BIS, see next section).

Neurobiologically, the FFFS is represented by the defence system (see Figure 3 for a visual representation), which comprises a set of structures modulated by serotonergic and noradrenergic inputs, and from the locus coeruleus and the raphe nuclei to different limbic and prefrontal regions (Cools, Nakamura, \& Daw, 2011; Deakin \& Graeff, 1991; Gray \& 
McNaughton, 2000; Jean-Richard-Dit-Bressel, Killcross, \& McNally, 2018; Lowry, Johnson, Hay-Schmidt, Mikkelsen, \& Shekhar, 2005; McNaughton \& Corr, 2004; McNaughton, DeYoung, \& Corr, 2016; Pickering \& Corr, 2008; Silva \& McNaughton, 2019; Smillie, 2008). Furthermore, this system is organised in a hierarchical psychobiological structure that encompasses specific avoidance behaviours related to the fear dimension and the brain regions aligned with these responses (Kennis, Rademaker, \& Geuze, 2013; McNaughton \& Corr, 2004, 2008, 2019). The main areas described for the FFFS are: the PAG, the medial hypothalamus, the amygdala, the anterior cingulate cortex (ACC), and the ventral prefrontal cortex. Together, these brain structures are responsible for the protection and integrity of the organism when coping with threatening stimuli.

Ethopharmacological experimentation in animals provided further evidence in relation to the functioning of this system. Specifically, the aforementioned structures were thought to work hierarchically based on the defensive distance (Blanchard \& Blanchard, 1990a; McNaughton \& Corr, 2004). From this perspective, the activation of the different brain areas involved in the defence system would depend on the proximity to a perceived threat. Thus, defensive avoidance behaviours would be driven by the PAG and medial hypothalamus at shorter distances (fight); intermediate distances would involve the amygdala (flight and freezing); and cortical areas (the anterior cingulate and ventral prefrontal cortices) would take control of behaviour at larger distances (non-defensive behaviours) (see McNaughton \& Corr, 2008). Then, within the different levels of the hierarchical defence system, the different brain structures, modulated by the serotonergic and noradrenergic systems, would promote appropriate responses to actively avoid aversive situations via a cascade of autonomic, endocrine, and motor responses (e.g. increased heart rate, blood pressure and release of stress hormones; Anderson \& Adolphs, 2014; LeDoux, 2000, 2014; LeDoux, Iwata, Cicchetti, \& Reis, 1988). 


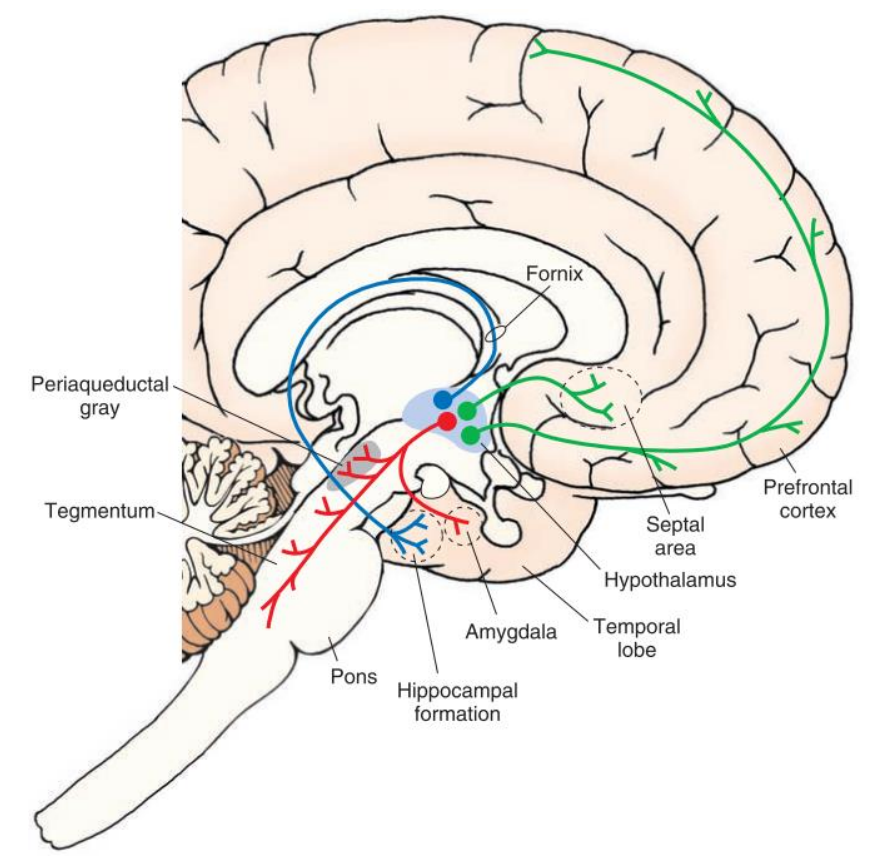

Figure 3. Graphical representation of the brain areas involved in the defensive system. Note that the regions depicted correspond to both the BIS and FFFS (the defence system, see text). Extracted from Siegel \& Sapru (2010).

\subsection{The Behavioural Inhibition System (BIS)}

The BIS was the cornerstone of the original RST model. However, new studies - mainly derived from animal experimentation - provided further evidence that refined and expanded previous concepts. In this way, the rRST proposed the BIS as the neurobehavioural system involved in resolving goal conflicts (Corr, 2004; Gray \& McNaughton, 2000). Thus, the BIS intercedes when a stimulus - conditioned or unconditioned - elicits activation in both the BAS and FFFS and there is a discrepancy between approach or avoid (this constitutes the paradigmatic approach-avoidance conflict). However, the BIS also resolves approach-approach (e.g. between two rewards) and avoidance-avoidance (between two punishments) conflicts. This state of uncertainty when resolving conflicts is associated with the subjective feeling of anxiety and a cognitive state of worry and rumination. Hence, the BIS initiates defensive approach behaviours toward the source of conflict, thus acting as a "comparator" by evaluating stimuli and context conditions (e.g. "risk assessment"; McNaughton \& Corr, 2018), and approach or avoidance responses would be elicited based on this evaluation.

Biologically, the septo-hippocampal system was classically set as the core of BIS (Gray, 1982), thus establishing the basis of anxiety as largely dependent on this single system (McNaughton \& Corr, 2004). However, thanks to prior work from the Blanchards and many 
others (e.g. Fanselow, 1991; LeDoux, 1994), the BIS was updated in order to adapt the theoretical framework to new evidence (Gray \& McNaughton, 2000). Thus, the rRST proposed the BIS as a neurobiological entity developed in parallel with the defence system (see Figure 3 for a schematic representation; Gray \& McNaughton, 2000; Jean-Richard-Dit-Bressel et al., 2018; McNaughton \& Corr, 2004, 2008, 2019; Pickering \& Corr, 2008). Similarly to the FFFS, the BIS also included serotonergic- and noradrenergic-irrigated structures such as the PAG, the medial hypothalamus, and the amygdala as the main regions within the system; nonetheless, the BIS also added the septo-hippocampal system, along with the posterior cingulate cortex (PCC) and the dorsal prefrontal cortex (to the detriment of the ACC and the ventral prefrontal cortex, respectively), as key regions within the system. All these brain structures were defined as the biological substrate explaining BIS activity and, by extension, Gray's anxiety.

As for the FFFS (see last section), the BIS was also dependent on the hierarchy of the defence system, stipulated as a function of the defensive distance (Blanchard \& Blanchard, 1990a; McNaughton \& Corr, 2004). Therefore, the activation of the brain regions involved in the BIS would depend on the perceived distance from conflicting stimuli (not only aversive situations per se). Therefore, defensive approach behaviours would be determined by the PAG and medial hypothalamus in shorter distances; intermediate distances would involve the amygdala and septohippocampal system; and cortical areas (the posterior cingulate and dorsal prefrontal cortices) would respond at larger distances (see McNaughton \& Corr, 2008).

\subsection{Interactions between systems}

Based on the rRST description, behavioural outcomes would be driven by the activation of the concurrent neurobehavioural system. Hence, BAS and FFFS activation promotes approach or defensive avoidance behaviours, respectively. Moreover, the activation of one of these systems inhibits the other, thus facilitating decision-making processes. In the presence of appetitive or aversive stimuli, BAS or FFFS engagement leads to motivated, vigorous behaviours aimed at obtaining rewards or avoiding punishments. In the absence of co-activation (i.e. clear unbalance between both systems), behaviours would be guided by the (more) activated system (Corr, 2008).

Nonetheless, when both systems are co-activated (simultaneous BAS and FFFS activation), a conflict situation arises. This conflict is resolved by means of the BIS by increasing the arousal and redirecting the attention to the stimuli that generate the conflict, also inhibiting preponderant BAS or FFFS behaviours. In this way, the BIS facilitates defensive approach behaviours toward the source of conflict, thus eliciting risk assessment and goal-resolving actions (McNaughton \& Corr, 2019). However, BIS activation is asymmetrical (there is a tendency to 
assess stimuli as aversive, due to the negative emotional bias and over-arousal generated by conflicting situations), thus favouring FFFS activation and, therefore, avoidance behaviours. However, the behavioural outcomes - and their intensity - rely on the systems that are activated in each moment. For instance, an avoidance behaviour will be less intense if there is some kind of reward in the context.

In summary, the rRST offers a comprehensive neurobehavioural description of approach/avoidance behaviours. Hence, studying the reactivity or level of activation of the different systems can give us valuable insight into the roots of specific adaptive and maladaptive behaviours.

\section{Personality dimensions from RST}

Gray's dimensions of personality were first elucidated to provide an alternative interpretation of Eysenck's introversion-extraversion and neuroticism-stability traits (Gray, 1970). These new personality dimensions, reward sensitivity and punishment sensitivity, were constructs linked to RST's impulsivity and anxiety. Importantly, these traits were considered the core of the BAS and FFFS/BIS activities (see Figure 4).

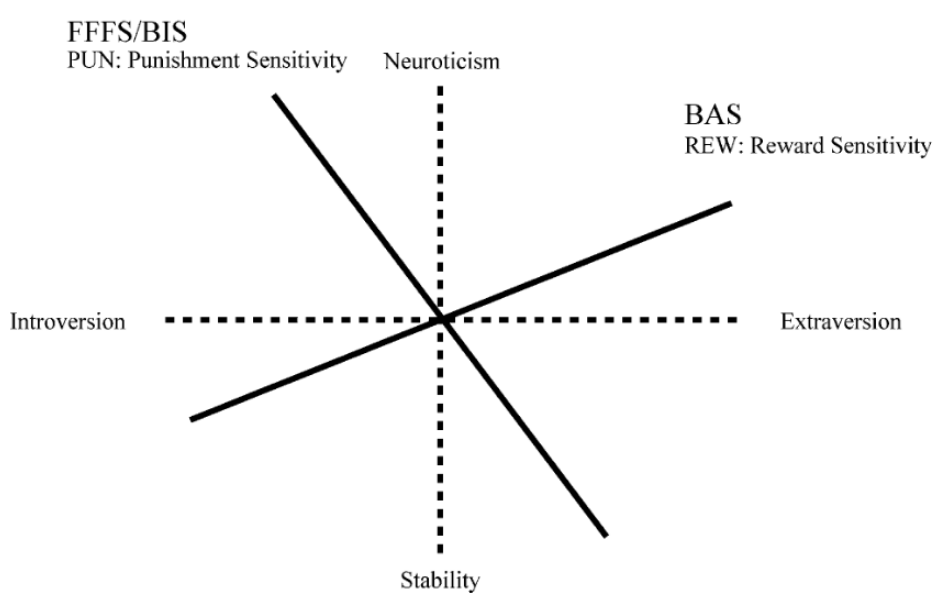

Figure 4. Relationship between rRST systems (BAS, BIS and FFFS) and personality dimensions (reward and punishment sensitivity) and their correspondence in the Eysenckian space (extraversion-introversion and neuroticism-stability traits). Extracted from Corr (2004).

In the following sections, we will discuss the cognitive and psychological aspects related to reward and punishment sensitivity. This discussion will offer a solid base with which to 
understand and delimit the characteristic behaviours of individuals with different reactivity in these systems.

\subsection{Reward sensitivity}

Sensitivity to reward (SR) was established as the personality trait producing individual differences in BAS activity (Gray, 1970, 1981, 1987a; Pickering et al., 1999). Due to its implication in a plethora of behaviours, this trait has been studied under laboratory conditions to discern its involvement in cognitive, affective, and motivational domains. Moreover, individual differences in this trait are thought to reflect a different sensitivity and reactivity of the BAS towards appetitive stimuli, that is, the way the BAS responds in the presence of rewards (sensitivity; "control mode") vs in the detection of rewards (reactivity; "checking mode") (Ávila \& Torrubia, 2008; Torrubia, Ávila, Moltó, \& Caseras, 2001).

In this way, previous behavioural studies have demonstrated that individuals with higher SR scores (BAS overactivation) show better learning in appetitive contexts, thus generating more intense and probable approach behaviours than low SR scorers (BAS underactivation) (see Ávila $\&$ Torrubia, 2008; Corr, 2008). Furthermore, these individuals over-focalise their attention on rewarding stimuli on decision-making tasks (Derryberry \& Reed, 1994), they show faster responses to obtain rewards in the presence of aversive cues (Avila, 2001), they show a preference for immediate rewards (Avila \& Parcet, 2000), and they show more perseverate responses to obtain rewards (Avila, Moltó, \& Segarra, 1995; Avila \& Parcet, 2000; Newman, Widom, \& Nathan, 1985). This tendency to engage in approach behaviours is engendered by a cognitive pattern: high reward sensitivity scorers over-focalise attention on locations where appetitive (or appetitive-related) stimuli are presented; they also disengage faster from previous stimuli, thus allowing a faster response to new stimuli (for a review, see Ávila \& Torrubia, 2008). This idea is supported by the dopaminergic function (Redgrave, Prescott, \& Gurney, 1999), given that new unexpected stimuli generate a short burst firing in the dopaminergic neurons that favours disengagement from previous stimuli to focus the attention on new stimuli. As a result, individuals who score high on this trait exhibit more reward-oriented behaviours, for example: more incorrect responses on a real multiple-choice exam (Ávila \& Torrubia, 2004), more food cravings (Franken \& Muris, 2006), more sexual experiences (Aluja, 2004), earlier drug consumption (Pardo, Aguilar, Molinuevo, \& Torrubia, 2007; Urošević et al., 2015), early entrepreneurial action (Lerner, Hatak, \& Rauch, 2018), and more deviant driving behaviours (Constantinou, Panayiotou, Konstantinou, Loutsiou-Ladd, \& Kapardis, 2011). 
Therefore, the general pattern associated with high reward sensitivity is a "hyperactive seeking" that leads to a higher probability of detecting salient, appetitive stimuli in the environment and vigorously approaching them once detected (even in the context of potential negative consequences).

\subsection{Punishment sensitivity}

Sensitivity to punishment (SP) is the personality trait proposed by the rRST as a source of variation in BIS and FFFS activity. Although two different systems would entail two different dimensions, this one-factor solution has been widely accepted because both systems share the same construct of defensive distance (McNaughton \& Corr, 2004; Pickering \& Corr, 2008). Thus, anxiety- and fear-related behaviours are neurally mediated by the perceived distance from threatening stimuli. Individual differences in SP introduce variance in this perception, so that high SP individuals tend to perceive aversive stimuli as closer than its "real distance", thus assessing more situations as perilous (McNaughton \& Corr, 2004, 2019). Additionally, the SP trait also reflects the sensitivity and responsivity levels of the BIS/FFFS in relation to aversive stimuli (i.e. differences in the control and checking modes, see the previous section).

Regarding the cognitive pattern, previous studies suggest that anxious individuals (overactive BIS/FFFS) are characterised by an attentional bias toward aversive stimuli and aversive-related locations compared to non-anxious individuals (hypoactive BIS/FFFS) (see Ávila \& Torrubia, 2008, for a review). These highly anxious individuals process threat-related stimuli more intensely (Fox, 1996; Mogg, Bradley, Williams, \& Mathews, 1993), exhibit higher distractibility due to neutral and aversive peripheral stimuli (Broadbent, Broadbent, \& Jones, 1986; Fox, 1994; Mathews, May, Mogg, \& Eysenck, 1990; Poy, Eixarch, \& Ávila, 2004), and show enhanced vigilance for aversive stimuli, such as threatening faces or pictures (Bradley, Mogg, Falla, \& Hamilton, 1998; Byrne \& Eysenck, 1995; Mogg, Garner, \& Bradley, 2007; Reinholdt-Dunne, Mogg, \& Bradley, 2009; Yiend \& Mathews, 2001). Moreover, this effect has also been replicated in children (Salum et al., 2013). All this evidence establishes a clear bias in anxious people that operates by narrowing the attentional focus on punishment-alerting signals. This attentional bias has recently been review by Mogg \& Bradley (2018). Thus, attentional mechanisms in these individuals are more easily triggered by threat cues (Mathews, Yiend, \& Lawrence, 2004; Mogg \& Bradley, 2002). As such, learning processes in aversive contexts are facilitated in individuals with higher anxiety-related traits. 
All in all, individuals with higher SP-related trait scores display a "hypervigilant" behaviour" biased toward cues that predict aversive stimuli, which facilitates the detection and response in the presence of punishments.

\subsection{RST measures}

One of the most important aspects when studying personality is to develop reliable measures to assess the theorised traits. Since the original publication of RST (Gray, 1981, 1982), different questionnaires have been designed with this aim. The most well-known questionnaires are the BIS/BAS scales (Carver \& White, 1994) and the Sensitivity to Punishment and Sensitivity to Reward Questionnaire (SPSRQ; Torrubia et al., 2001). On the one hand, the BIS/BAS scales consist of 20 Likert-type items organised in different subscales; one for measuring BIS activity (7 items) and three for assessing BAS functioning, including Drive (4 items), Fun Seeking (FS; 4 items), and Reward Responsiveness (RR; 5 items). The BIS scale includes items for measuring anticipatory reactions to aversive stimuli; Drive assesses the persistence in obtaining desired goals; FS represents desirability for new rewards and the predisposition to approach them on impulse; and RR evaluates behavioural responses to the occurrence or expectancy of rewarding stimuli.

On the other hand, the SPSRQ is composed of 48 "Yes-No" items; 24 for SP and 24 for SR. The SP scale was intended to assess behavioural inhibition in uncertain situations (i.e. in contexts where an individual can receive a punishment) and the worry or cognitive distress produced by the threat of aversive stimuli. The SR scale was intended to evaluate and describe typical approach behaviours toward rewarding high-incentive stimuli such as money, drugs, sex, social interactions, etc. (Torrubia, Avila, \& Caseras, 2008). These two questionnaires have been widely used in a large number of studies from different areas (i.e. psychology, neuroscience, pharmacology, genetics, etc.) - indeed, both have been officially translated into more than 15 languages. It is noteworthy, though, that there were other different attempts to evaluate the personality dimensions of the RST. These scales are summarised in Torrubia, Avila, \& Caseras (2008): the Susceptibility to Punishment scale (Torrubia \& Tobeña, 1984), the Gray-Wilson Personality Questionnaire (Wilson, Barrett, \& Gray, 1989), the General Reward and Punishment Expectancy scales (Ball \& Zuckerman, 1990), the BIS scales (MacAndrew \& Steele, 1991), and the Appetitive Motivational scale (Jackson \& Smillie, 2004).

Psychometrically, all the aforementioned scales show good internal consistency as well as adequate convergent and discriminant validity (see Torrubia et al., 2008). However, some important differences are observed between the BIS and BAS: whereas all the BIS measures show 
strong correlations with each other, the correlations between the BAS scales are low to moderate. Moreover, the BIS scales are highly related to different anxiety-like measures (i.e. the Neuroticism trait from the Eysenck Personality Questionnaire (EPQ; Eysenck \& Eysenck, 1975), Harm Avoidance from the Tridimensional Personality Questionnaire/Temperament and Character Inventory (TPQ/TCI; Cloninger, 1987; Cloninger, Svrakic, \& Przybeck, 1993), or the State-Trait Anxiety Inventory (STAI-T; Spielberger, Gorsuch, \& Lushene, 1970), whereas the BAS scales show significant, but weaker, relationships with impulsivity-related scales (i.e. Extraversion from the EPQ, Novelty Seeking from the TPQ, or the $I_{7}$ Impulsiveness Questionnaire (Eysenck, Pearson, Easting, \& Allsopp, 1985)). Some of these results were previously described in Caseras, Àvila, \& Torrubia (2003).

Despite the number of questionnaires, the BIS/BAS scales and the SPSRQ have been as the most widely used measures for assessing RST system activity. Indeed, these are the only two questionnaires with test-retest reliability reports - showing acceptable consistency (Torrubia et al., 2008). However, there are some differences between the two scales. In particular, although both the BIS and SP scales show high, positive correlations with Neuroticism and negative correlations with Extraversion, this latter correlation is higher (more negative) for SP. On the other hand, the BAS and SR scales are positively associated with Extraversion, but a different pattern is observed for Neuroticism: the BAS scale shows a low positive association (close to zero); however, SR presents a higher positive correlation. This latter finding resembles the pattern observed for SR with Eysenck's impulsivity dimension (i.e.: "neurotic extraversion").

However, although these measures (e.g. the BIS/BAS scales and the SPSRQ) were adequate to assess the dimensions derived from the original RST, the distinction between the FFFS and BIS carried out in the rRST (Gray \& McNaughton, 2000; McNaughton \& Corr, 2004) fostered the development of new questionnaires designed to assess fear and anxiety separately. Indeed, there is little doubt that, although related (and overlapped in some aspects), fear and anxiety encompass different dimensions (Perkins, Kemp, \& Corr, 2007; Sylvers, Lilienfeld, \& LaPrairie, 2011). This new set of questionnaires includes: the Jackson-5 (Jackson, 2009), the Reinforcement Sensitivity Questionnaire (RSQ; Smederevac, Mitrović, Čolović, \& Nikolašević, 2014), the revised Reinforcement Sensitivity Theory Questionnaire (rRST-Q; Reuter, Cooper, Smillie, Markett, \& Montag, 2015), and the Reinforcement Sensitivity Theory of Personality Questionnaire (RST-PQ; Corr \& Cooper, 2016). In addition, Colder et al. (2011) developed a new measure in children based on a factor analysis of the SPSRQ. Finally, a clinical-oriented questionnaire designed to assess the Fight, Flight and Freeze components has been elaborated (Maack, Buchanan, \& Young, 2015). A detailed description of these questionnaires with recommendations can be found in Corr (2016). 
Even so, it is important to note that, although the first scales (e.g. the BIS scale and SP) were not intended to assess the BIS and FFS independently, this solution was adopted based on the theoretical framework of the old RST. Actually, in the case of the SPSRQ, it is plausible that SP captures both the fear and anxiety dimensions in a higher order domain of negative emotionality (Corr, 2004; McNaughton \& Corr, 2004; Pickering \& Corr, 2008), reflecting individual differences in the defensive distance.

\subsection{RST and psychopathology}

Taking into consideration the cognitive patterns linked to SR and SP described in the previous sections, it is plausible, then, to assume a relationship between these traits and clinical manifestations. Indeed, the existing literature relating RST and psychopathology is extensive (for a summary on this topic, see Bijttebier, Beck, Claes, \& Vandereycken, 2009). In this context, different levels of activation in the RST systems have been associated with a number of clinical conditions, thus providing valuable insights into psychopathological vulnerability in healthy individuals.

Regarding BAS, RST has related high activity in this system to a number of disinhibitory disorders, which are characterised by deficient impulse control, such as substance use and abuse (Balconi, Finocchiaro, \& Campanella, 2014; Boog et al., 2014; Franken, Muris, \& Georgieva, 2006; Kimbrel, Nelson-Gray, \& Mitchell, 2007; Knyazev, 2004; Loxton \& Dawe, 2006; O'Connor, Stewart, \& Watt, 2009; Pardo et al., 2007; Simons, Dvorak, \& Batien, 2008; Yen et al., 2012), attention-deficit/hyperactivity disorder (ADHD; Mitchell \& Nelson-Gray, 2006; Newman \& Wallace, 1993; Nigg, 2001), psychopathy (Hundt, Kimbrel, Mitchell, \& Nelson-Gray, 2008; Kimbrel et al., 2007; Newman, MacCoon, Vaughn, \& Sadeh, 2005; Ross, Benning, Patrick, Thompson, \& Thurston, 2009; Ross et al., 2007; Uzieblo, Verschuere, \& Crombez, 2007), eating disorders (Beck, Smits, Claes, Vandereycken, \& Bijttebier, 2009; Glashouwer, Bloot, Veenstra, Franken, \& de Jong, 2014; Kane, Loxton, Staiger, \& Dawe, 2004; Matton, Goossens, Braet, \& Vervaet, 2013; Matton, Goossens, Vervaet, \& Braet, 2015), bipolar disorder (Alloy \& Abramson, 2010; Alloy et al., 2012; Salavert et al., 2007), cluster B personality disorders (Pastor et al., 2007; Taylor, Reeves, James, \& Bobadilla, 2006), and even more recent behavioural addictions, such as Internet gambling disorder (Farrell \& Walker, 2019; Na, Lee, Choi, \& Kim, 2017). In addition, lower activity in this system has been related to depression (Hundt, Nelson-Gray, Kimbrel, Mitchell, \& Kwapil, 2007; Kasch, Rottenberg, Arnow, \& Gotlib, 2002; Pinto-Meza et al., 2006).

In relation to the BIS, previous studies have also associated the reactivity of this system with clinical symptoms. In particular, an overactive BIS has been linked to anxiety disorders and 
anxiety-related symptoms (Hundt et al., 2007; Kimbrel, 2008; Maack, Tull, \& Gratz, 2012; Sportel, Nauta, de Hullu, de Jong, \& Hartman, 2011; Struijs et al., 2018; Vervoort et al., 2010). By contrast, BIS hypoactivation is associated with psychopathy (Fowles, 1980; Kimbrel et al., 2007; Newman et al., 2005; Ross et al., 2009, 2007).

Finally, although the association between the FFFS and psychopathology has been studied less, prior research posited that this system would also be related to anxiety disorders, but more specifically those with an emotional fear component, such as panic, phobias, or obsession (Barlow, 1988; Kimbrel, 2008; Pickering \& Corr, 2008). It is worth noting, though, that this lack of evidence from experimental testing may stem from the absence of proper measures. However, new instruments specifically designed to assess FFFS activity, such as the Fight, Flight, Freeze Questionnaire (Maack et al., 2015), would be useful for this aim.

Overall, the extensive literature on the RST systems and their relationship with psychopathology highlights the relevance of this topic when studying human behaviour. Indeed, in light of this evidence relating personality and psychopathology, we can consider personality traits in their extreme forms to be a "warning sign" of a possible predisposition or vulnerability to clinical disorders.

\section{Brain basis of RST}

The biological description was one of the strongest points of RST. In fact, the theoretical constructs depicted in the original model (Gray, 1981, 1982, 1987a) and in the rRST (Gray \& McNaughton, 2000; McNaughton \& Corr, 2004) were supported by extensive animal literature. Nonetheless, less evidence exists on the brain correlates in humans. Undoubtedly, Gray's model has made a major contribution to the psychology of personality - in fact, there is a vast body of literature relating RST personality dimensions to behavioural traits - and yet it is necessary to carry out new research that relates the pre-existing non-human evidence to human brain data. Furthermore, by studying the brain anatomical and functional correlates of RST in the human brain, we could better understand human personality and its influence on the cognitive, affective, and behavioural domains, thus developing - an even richer - framework with which to study normal and abnormal behaviours. Moreover, if the rRST assumptions are confirmed in humans, we could use these data to predict, based on individual differences in personality, a psychopathological predisposition in humans, that is, a "red flag" for individuals at risk of developing a clinical condition, from a neurobiological perspective. 


\subsection{Evidence from neuroimaging}

Is it possible to confirm the neurobiological findings of RST (based on animal models) in human beings? This could be the starting point for studies carried out in humans by means of MRI. Although the neurobiological description of the RST systems was undoubtedly valuable, new neuroimaging techniques can help to further explain and expand the model in humans. Moreover, even in the early 2000s when the rRST came out, the use of MRI techniques was in its infancy. Fortunately, MRI research has advanced considerably in the past 20 years, so that currently we have an excellent set of toolboxes available to explore the brain in detail - both functionally and anatomically. However, due to the recent implementation of MRI techniques, only a few studies have studied the association between BAS and BISF/FFS activation and the brain structure and function. Nevertheless, these early attempts have provided the first evidence and confirmation of the brain basis of RST in humans. In the following sections, we are going to summarise the main MRI findings reported for the BAS (reward system) and BIS/FFFS (punishment/defensive system).

\subsection{BAS correlates}

\subsubsection{Structural MRI studies}

Different studies have provided evidence about BAS activity and the regional grey matter volume (GMV) of reward-related areas. To this aim, different measures of anatomy, such as voxel-based morphometry (VBM) or cortical surface (cortical thickness, CT), have been utilised, as well as structural connectivity by diffusion tensor imaging (DTI).

As for VBM, only two previous studies have reported associations between GMV and BAS activity. Barrós-Loscertales et al. (2006) first observed a GMV reduction in prefrontal (superior frontal cortex) and striatal (caudate, putamen and pallidum) areas associated with higher scores on the SR scale (overactive BAS). However, this study was carried out in a small sample of male participants. In another study conducted in a large sample using the BAS scale (Yadan Li et al., 2014), differences between the sexes (with males reporting lower GMV and females reporting higher GMV) were found in the ventromedial prefrontal cortex (vmPFC) and inferior parietal lobule (IPL), but this study did not explore the structural correlates in the whole sample. These results using specific BAS measures have been complemented by research using BASrelated traits, such as the NS scale from the TPQ/TCI by Cloninger. For example, in a recent study conducted in males, Caravaggio et al. (2017) found a negative correlation between NS and GMV 
in the putamen. However, the relationship between NS and GMV in the prefrontal cortex has produced mixed results. Previous studies have found a positive association between NS and the GMV in the superior and middle frontal gyri (SFG/MFG; Gardini, Cloninger, \& Venneri, 2009; Iidaka et al., 2006), whereas others have reported a negative correlation in the inferior frontal gyrus (IFG; Van Schuerbeek, Baeken, De Raedt, De Mey, \& Luypaert, 2011). Likewise, other studies have reported associations with different brain areas: the posterior cingulate cortex (PCC; Gardini et al., 2009; Van Schuerbeek et al., 2011), the superior temporal gyrus (STG; Stam, Huang, \& Van den Stock, 2019), and the cerebellum (Picerni et al., 2013; Van Schuerbeek et al., 2011). It is noteworthy, however, that all these studies have been conducted with relatively small sample sizes (less than 100 subjects), which could account for the differences observed across the studies, thus limiting further interpretations. Interestingly, a recent study conducted in a large sample $(\mathrm{N}=1050)$ reported $\mathrm{CT}$ reductions linked to a composite measure of sensation-seeking (including the BAS scale and NS; see Holmes, Hollinshead, Roffman, Smoller, \& Buckner, 2016). The brain areas showing a reduced CT were mainly located in the anterior cingulate cortex (ACC) and the MFG.

With regard to structural connectivity, only two previous investigations have linked BASrelated traits to DTI-derived measures. In these studies, a positive correlation was found between striatal connectivity and NS. In particular, white matter fibre connectivity from prefrontal areas (the medial and lateral orbitofrontal cortices) to the striatum were positively correlated with NS scores (Lei et al., 2014). Likewise, fibre tracts connecting the amygdala with the striatum were found to be stronger in individuals with high NS. In line with this latter result, Cohen, SchoeneBake, Elger, \& Weber (2009) also found a positive association between NS and tract strength between the amygdala/hippocampus and the striatum. This evidence from DTI studies supports the connection between medial prefrontal regions and the basal ganglia and its relationship with personality traits (e.g. NS). Along with the amygdala, these brain areas form a well-established cortico-basal ganglia loop that is more strongly connected in participants with higher NS.

\subsection{2 fMRI studies}

A number of studies have also reported evidence about the relationship between BAS measures and brain activity in reward-related areas (a review on this topic can be found in Kennis, Rademaker, \& Geuze, 2013), thus supporting the correspondence between the activity of this system and the functioning of the cortico-basal ganglia circuit. In other words, the processing of reward cues activates the brain areas involved in the BAS. 
On the one hand, some investigations have focused on analysing brain function by using task-based fMRI. Thus, different reward-related paradigms (i.e. using food or erotic images, monetary incentives, etc.) have been applied to simulate situations involving positive contingencies. These studies have consistently associated higher BAS activity with greater activation of the brain areas involved in the mesolimbic dopaminergic pathway (e.g. the midbrain and the ventral striatum). Beaver et al. (2006) first reported a positive correlation between brain activity in the midbrain and the ventral striatum elicited by food images and scores on the BAS scale. In this line, subsequent studies found similar results by positively linking BAS traits with higher activity in the VTA and NAcc during the anticipation of monetary gain cues (Carter, Macinnes, Huettel, \& Adcock, 2009; Costumero, Barrós-Loscertales, Bustamante, VenturaCampos, Fuentes, \& Ávila, 2013). This positive relationship between reward anticipation and processing and activation of the NAcc in individuals with an overactive BAS has been further reported by using similar monetary paradigms (Costumero et al., 2016; Hahn et al., 2009; Simon et al., 2010) and erotic pictures (Costumero, Barrós-Loscertales, Bustamante, Ventura-Campos, Fuentes, Rosell-Negre, et al., 2013). Furthermore, higher activity in the NAcc was also found using NS (see Abler, Walter, Erk, Kammerer, \& Spitzer, 2006). Concurrently, higher activation of ventromedial prefrontal areas (e.g. the mesocortical pathway) during the processing of appetitive stimuli has been pointed out (Barrós-Loscertales et al., 2010; Beaver et al., 2006; Costumero, Barrós-Loscertales, Bustamante, Ventura-Campos, Fuentes, Rosell-Negre, et al., 2013; Hahn et al., 2009; Locke \& Braver, 2008; Simon et al., 2010). Moreover, BAS scores have also been correlated with striatal and prefrontal activity during executive tasks (Avila et al., 2012; Fuentes-Claramonte et al., 2016b, 2016a, 2015; Fuentes et al., 2014b, 2014a), thus establishing a link between cognitive and motivational processes.

On the other hand, functional connectivity (FC) between reward-related areas has been less explored. Costumero, Barrós-Loscertales, Bustamante, Ventura-Campos, Fuentes, \& Ávila (2013) reported a psychophysiological interaction (PPI) between the VTA-SN and the medial orbitofrontal cortex (mOFC), showing higher connectivity between the two structures in high SR individuals during low incentive stimuli. This result indicates that, in the absence of potential cues predicting rewarding stimuli, the coupling between the VTA-SN and the mOFC is stronger, thus establishing a behavioural pattern aimed at seeking new incentives. In line with this study, higher NS has been associated with stronger FC between salience network areas when expecting lowsalient stimuli (Li et al., 2017). In addition, in the only resting-state study using the BAS scale, a positive correlation was found between Fun Seeking and the middle orbitofrontal cortex-putamen FC, whereas Drive was negatively associated with the FC between the middle cingulate cortex and the caudate nucleus (Angelides, Gupta, \& Vickery, 2017). Together, these previous studies show that low expectancy conditions (or the complete absence of stimuli) set up a brain state in 
"checking mode", where the mesocorticolimbic system, modulated by dopaminergic transmission, promotes seeking actions that facilitate the detection, evaluation, and posterior learning of salient events predicting potential rewards (Bromberg-Martin et al., 2010; Pickering \& Gray, 2001; Richter \& Gruber, 2018). This cognitive state would be linked to a higher FC between the brain areas involved in the dopaminergic reward system.

\subsubsection{BAS: neurobiological overview}

Based on cumulative evidence from MRI, we can conclude that the RST premises are generally confirmed in humans. The BAS describes an entire neuropsychological system involved in detecting and promoting preponderant behaviours to obtain rewards, regardless of the origin. BAS-related behaviours have been tested in different task situations by using a variety of appetitive stimuli, such as monetary incentives, social recognition, food, or sex (Pickering \& Gray, 2001). Hence, appetitive stimuli activate the BAS, which in turn initiates a cascade of motivated behaviours that help to lead the organism to rewarding stimuli (McNaughton \& Corr, 2008). The dopaminergic system plays a major role in this process (Pickering \& Gray, 2001). Within this system, the main region is the VTA, which is connected to the NAcc and prefrontal regions via the mesolimbic and mesocortical pathways, respectively (Arias-Carrión et al., 2010; Düzel et al., 2009; Haber, 2014).

Interestingly, studies in humans have shown that stable, well-defined, individual differences in behavioural approach traits underlie variances in the structure and function (and FC) of the brain areas involved in this system (see previous sections). Thus, similarly to rodents, the midbrain (i.e. the VTA), the striatum (i.e. the NAcc and caudate), and the frontal lobe (including the cingulate cortex) have been proposed as the core regions of the BAS in humans.

Nonetheless, even though the studies conducted so far have provided relevant insights into RST and human personality, some aspects remain unclear. In particular, only a few studies have analysed the cortical and subcortical GMV linked to specific BAS measures (i.e. the SR scale from the SPSRQ or the BAS scale from the BIS/BAS scales), and the underlying sex differences are also unexplored. Furthermore, although previous studies have reported functional correlates, evidence on FC is scarce, particularly when studying the brain "at rest". Therefore, future studies should address these issues by conducting new research with larger samples that add support to previous research, in order to better understand how individual differences are related to the brain structure and function. 


\subsection{BIS correlates}

\subsubsection{Structural MRI studies}

Different studies have reported a relationship between BIS activity and different structural measures (i.e. GMV, DTI, or cortical surface) (see Mincic, 2015, for a review). Anatomical studies have reported GMV increases in limbic areas in individuals with high scores on BIS measures (the BIS scale from the BIS/BAS scales and SP from the SPSRQ). In this way, Barrós-Loscertales et al. (2006a) first reported a higher GMV in the amygdala and hippocampus in a small sample of male participants. Nonetheless, subsequent studies only found GMV increases in the hippocampus (Cherbuin et al., 2008; Levita et al., 2014). However, these studies were conducted in a reduced sample of males and females (Levita et al., 2014), or they focused on a specific age-range (see Cherbuin et al., 2008) that differed considerably from the sample used in Barrós-Loscertales et al. (2006a). This discrepancy between methodological aspects, among others, could account for the differences observed between these studies. It is noteworthy, however, that in a study conducted in a large sample $(\mathrm{N}=1050)$ using composite measure of negative affect traits (including the STAI-T, Neuroticism, the BIS scale, HA, and mood disturbance), a positive association was found between the amygdala and hippocampus GMV and the scores on this scale (Holmes et al., 2012). In addition, studies using HA from the TCI and Neuroticism from the NEO-FFI (Costa \& McCrae, 1992) have supported this association (Iidaka et al., 2006; Koelsch, Skouras, \& Jentschke, 2013; Kyeong, Kim, Park, \& Hwang, 2014). At the cortical level, GMV reductions have been found in the precuneus and the OFC in individuals with high BIS activity (Fuentes et al., 2012). Similar results were obtained in other studies using HA (Gardini et al., 2009) and Neuroticism (Delaparte et al., 2019). As for surface metrics, previous studies using Neuroticism found a negative correlation between the CT and surface area (SA) of prefrontal areas (including the frontal pole, the mOFC, and the ACC) and the scores obtained on this scale (Bjørnebekk et al., 2013; Wright et al., 2006). This reduction in the mOFC and ACC was also observed in the study by Holmes et al. (2012).

No previous DTI studies have been carried out with the BIS scale or SP. However, in a prior investigation using a trait anxiety score (a composite measure derived from HA, Neuroticism, and BIS), Montag, Reuter, Weber, Markett, and Schoene-Bake (2012) found a positive association with the hippocampus-cingulum tract integrity. Similarly, a higher integrity of the cingulum tract was also found in Madsen et al. (2012) in individuals with high Neuroticism. Likewise, HA has been associated with higher connectivity between the striatum and hippocampus (Cohen et al., 2009), whereas the former has also been associated with the prefrontal and posterior cingulate cortices (Lei et al., 2014). 


\subsection{2 fMRI studies}

Functional studies have produced consistent results through task-based fMRI conditions. Particularly, BIS activity has been positively associated with the amygdala and the cingulate cortex when processing negative stimuli (i.e. viewing fearful faces/pictures; Cools et al., 2005; Reuter et al., 2004). These findings have been further replicated in a number of studies using different BIS-related traits in similar negative affective conditions. Thus, greater activation of the amygdala has been linked to higher scores on anxiety traits (Etkin, Egner, Peraza, Kandel, \& Hirsch, 2006; Ewbank et al., 2009; Frühholz, Prinz, \& Herrmann, 2010; Krug \& Carter, 2010) and HA (Baeken et al., 2009). Interestingly, studies using Neuroticism have also reported a positive correlation with cingulate and prefrontal regions (Canli et al., 2001; Cremers et al., 2010; Cunningham, Arbuckle, Jahn, Mowrer, \& Abduljalil, 2010). For a review on this topic, see Kennis et al. (2013).

In terms of connectivity, only one previous study utilised BIS specific measures (i.e. SP) to analyse the FC between punishment-related areas. This study observed higher connectivity between the amygdala and the hippocampus following signs of potential monetary loss (Hahn et al., 2010). In this line, different investigations using BIS-related traits (trait anxiety, Neuroticism, or HA) also found a direct relationship between amygdala FC and scores on these traits. These studies found correlations between amygdala FC and distinct brain areas, mainly cortical (the prefrontal cortex, the precuneus, or the cingulate) (Aghajani et al., 2014; Baeken et al., 2014; Buckholtz et al., 2008; Cremers et al., 2010; Gentili et al., 2017; Kim, Gee, Loucks, Davis, \& Whalen, 2011; Ying Li, Qin, Jiang, Zhang, \& Yu, 2012; Pang et al., 2016). However, some of these investigations have reported contradictory results, and so further research is needed in order to better understand the FC dynamics linked to BIS traits.

\subsubsection{BIS/FFFS: neurobiological overview}

As for the BAS, neuroimaging studies have provided evidence in humans that highlight the influence of limbic areas, which form the core of the defence system, on behavioural inhibition, thus confirming the brain basis of RST in humans.

It is important to point out, though, that the defence system works hierarchically; that is, different structures take control of behaviour based on the defensive distance. Thus, "lower-order" regions (the amygdala, the PAG, and the hypothalamus) would promote fast responses aimed at protecting the organism immediately, whereas "higher-order" structures (the hippocampus and 
prefrontal regions) would inhibit possible risk behaviours (for recent reviews, see McNaughton \& Corr, 2018, 2019) when danger is not present. Interestingly, individual differences in behavioural inhibition personality traits are related to these areas. Currently, there is abundant literature relating amygdalar and hippocampal structures and functions with these traits. However, no previous studies have investigated the association of the lower hierarchy structures (i.e. the PAG or the hypothalamus) with personality traits.

Hence, future studies should take these structures into account in order to provide complete confirmation of RST in humans. 


\section{CHAPTER 2. EXPERIMENTAL SECTION}

\section{Overview of the investigation}

Personality influences one's habitual behaviour. As such, personality traits can be studied in order to obtain reliable and feasible measures that explain and predict future behaviours. This is possible thanks to previous extensive work aimed at linking these traits to specific behaviours, thus relating behavioural patterns to distinct personality dimensions. However, individual differences in personality cannot be fully understood without a concise description at the biological level. Stable differences in the functioning of the underlying neurobiological systems (e.g. the brain reward and punishment circuits) encompass differences in personality traits (DeYoung, 2010). Thus, the first comprehensive models of personality (e.g. Eysenck's and Gray's models) were developed within a psychobiological framework. Therefore, RST (Gray, 1970, 1981) proposed a comprehensive neuropsychological model to describe and predict behaviours based on a biological predisposition. Nonetheless, since the publication of the rRST (Gray \& McNaughton, 2000), personality psychology has neglected the biological study of personality traits in favour of a cognitive neuroscience approach. Whereas this approach has provided valuable insights in terms of cognitive functioning (for example by analysing the differential response to emotional stimuli), there is little evidence that relates reward and punishment sensitivity with the brain's default architecture - that is, with stable structural and resting-state brain patterns. Therefore, by integrating this evidence, we could better understand how the brain is anatomically and functionally shaped, and how it operates in the absence of stimulation (i.e. representing the "brain's default state"). In this way, brain differences at this level could account for the differential - based on personality traits - response observed when people deal with rewards and punishments.

Therefore, even though the biological description of RST - based on animal research has been thorough, its correspondence to humans is not yet completely understood. It is clear, however, that the neurobiological systems depicted in RST are equally represented in the human brain (e.g. the reward and punishment systems). The reward system is paralleled by the dopaminergic circuitry (Berridge, 2007; Berridge \& Robinson, 1998; Cools, 2008; Haber \& Knutson, 2010; Hayes et al., 2014; Ikemoto, 2007; McClure, York, \& Montague, 2004; Sescousse, Caldú, Segura, \& Dreher, 2013; Wise, 2004). Likewise, RST proposes this system as the core of the BAS (McNaughton \& Corr, 2008; Pickering \& Corr, 2008), so that individual differences in BAS functioning (higher or lower reactivity of the brain areas involved in this system) generate stable, measurable individual differences in the personality dimension of reward 
sensitivity. Thus, variability in this trait would establish a different brain configuration when detecting and responding to reward stimuli. Similarly, the punishment system has been widely described in humans (Gray \& McNaughton, 2000; Hayes et al., 2014; Jean-Richard-Dit-Bressel et al., 2018; McNaughton \& Corr, 2004, 2018; Pickering \& Corr, 2008). This system involves two different defensive directions - approach (BIS) and avoidance (FFFS) - represented by a set of overlapping serotonin-irrigated structures that work hierarchically. This hierarchy is analogous to the defensive distance, that is, activation of higher- or lower-order structures depends on the perceived distance from the threatening stimulus, whose perception varies as a function of individual differences in SP (McNaughton \& Corr, 2019). This variability alters the perceived distance, thus setting a higher or lower probability of engaging in defensive behaviours. Thus, by analysing the brain structural and functional correlates associated with SR and SP, we can better describe the brain basis of the BAS and BIS/FFFS in humans and the brain characteristics that lead to extreme forms in these personality traits.

Currently, MRI offers useful means for studying the human brain in vivo. In addition, MRI makes it possible to obtain, non-invasively, brain structural and functional measures with great spatial resolution and acceptable temporal resolution. This non-invasive nature makes MRI preferable to other neuroimaging techniques, such as CAT (computerised axial tomography), PET (positron emission tomography), or SPECT (single-photon emission computerised tomography). For this reason, MRI has become very popular in the past 20 years within personality neuroscience, given that it is a very useful method to test neuropsychological constructs, such as those described by RST. However, although some studies have been carried out to confirm the theorised constructs in humans by means of MRI studies, the existing literature is scarce.

Even though previous studies have reported brain structural correlates consistent with the endophenotypes described in the model for the BAS, BIS, and FFFS, some differences arise across studies. On the one hand, GMV and CT reductions in medial prefrontal areas have been observed in participants with higher scores on BAS-related measures (Barrós-Loscertales et al., 2006b; Holmes et al., 2016), whereas Barrós-Loscertales et al. (2006b) additionally reported lower striatal volume associated with an overactive BAS in males. On the other hand, previous research has shown a GMV increase in brain areas linked to the BIS/FFFS, such as the amygdala (Iidaka et al., 2006), the hippocampus (Cherbuin et al., 2008; Levita et al., 2014), or both (BarrósLoscertales et al., 2006a; Holmes et al., 2012). Thus, although all these studies have demonstrated the relationship between frontostriatal and limbic areas and BAS and BIS/FFFS activity, respectively, there are some discrepancies between studies. In the first study included in this thesis, we addressed this issue by using a large sample $(\mathrm{N}=400)$ of unmedicated participants in order to analyse the relationship between these structures (e.g. the amygdala, the hippocampus, and the striatum) and the personality traits depicted in RST. 
Although a large number of investigations have studied the relationship between RST traits and brain function, they have predominantly focused on task-based contingency paradigms (both reward- or punishment-based; see Kennis et al., 2013), whereas little evidence exists about the brain function at rest, that is, in the absence of stimuli. This stimulus-free context allows us to study the spontaneous fluctuations in the BOLD signal, which in turn represents intrinsic brain patterns. These functional patterns observed during resting-state are a reliable measure of stable brain differences over time (Abrol et al., 2017; Choe et al., 2015; Shehzad et al., 2009). In this way, brain activity at rest may reflect individual differences that come with experience. That is, cognitive biases and personal traits establish characteristic brain patterns that can be captured by the measurement of spontaneous fluctuations (Harmelech \& Malach, 2013). Therefore, by analysing the brain dynamics during a resting-state condition (e.g. in functional connectivity), we could observe stable functional differences linked to personality traits (in our case, to reward and punishment sensitivity traits) that may describe a behavioural predisposition -for instance, in taskbased paradigms. Indeed, the intrinsic brain activity can be decomposed into different components (i.e. functional networks) that mimic typical task-based activity (Raichle, 2015; Smith et al., 2009).

Since its original conception (Biswal, Zerrin Yetkin, Haughton, \& Hyde, 1995), the use of the resting-state as a paradigm for studying brain functional connectivity has grown significantly, producing a number of studies using different approaches (van den Heuvel \& Hulshoff Pol, 2010). Resting-state functional connectivity (rsFC) between "seed" regions is one of them. This approach is especially useful when studying rsFC between a priori regions of interest (ROIs), that is, ROIs selected based on previous hypotheses. So far, only three previous studies have investigated the connectivity between ROIs associated with the reward and punishment systems and their influence on BAS and BIS/FFFS measures. On the one hand, Hahn et al. (2010, 2013) showed a stronger FC between the amygdala and the hippocampus in high SP individuals, thus providing evidence about the functional connectivity of punishment-related areas and its influence on BIS/FFFS traits. It must be noted, however, that this effect was observed during a task condition (anticipation of monetary loss). On the other hand, Angelides et al. (2017) reported a positive correlation between BAS fun-seeking and the rsFC between the putamen and the OFC, whereas a negative correlation was found between BAS drive and the rsFC between the caudate and the middle cingulate cortex. However, these two studies should be interpreted with caution. Although they provide valuable insights into personality and brain connectivity, the first two studies were carried out by using a task-based fMRI paradigm, thus restricting the interpretation to stimuli-dependent contexts, whereas in the latter study, no specific BAS-related areas such as the NAcc or the caudate were studied. Therefore, new studies are needed to further explore the functional connectivity of the brain areas included in the reward and punishment 
systems and its influence on RST traits. Moreover, by studying the functional connectivity of these areas during a resting-state condition, we are able to observe the brain configuration of these systems in the absence of stimuli, thus contrasting previous fMRI task-derived reports. Study 2 and Study 3 from this thesis were especially designed with this aim. We conducted these studies to see if the rsFC between the vmPFC, ACC, NAcc, and VTA influenced the appearance of individual differences in SR, whereas we performed the same analyses with the amygdala, hippocampus, and PAG and the SP trait. As in the case of Study 1, Study 2 and Study 3 were also carried out in large samples ( $\mathrm{N}=128$ and $\mathrm{N}=198$, respectively) of unmedicated healthy young adults.

All in all, in this thesis we have employed different MRI techniques in order to confirm RST in humans - thus extending previous evidence from animal models. So, by using structural and rsFC measures, we conducted three studies; Study 1 was aimed at describing the structural correlates linked to SR and SP, whereas Study 2 and Study 3 were focused on the rsFC of the main areas involved in the reward and punishment systems and the relationship with SR and SP.

\section{Objectives and hypotheses}

The overall objective of this thesis is to provide new evidence about the personality traits derived from RST and their brain correlates in the human brain. We performed three different studies in order to investigate stable brain patterns (e.g. in brain structure and functional connectivity at rest) in large samples of healthy young adults. In particular, the studies included in this thesis were aimed at confirming the relationship between the SR and SP traits and the GMV of reward- and punishment-related areas, respectively, as well the rsFC of these areas and the association with the same personality traits. The specific goals of this investigation consist of analysing the relationship between:

1. Reward sensitivity and the grey matter volume at both whole-brain and region-ofinterest (focused on striatal areas) levels.

2. Punishment sensitivity and the grey matter volume at both whole-brain and regionof-interest (focused on limbic areas) levels.

3. Reward sensitivity and the functional connectivity at rest between reward-related areas.

4. Punishment sensitivity and the functional connectivity at rest between punishmentrelated areas. 
Based on these objectives and the revised literature, we formulate the following hypotheses:

1. There will be a negative correlation between SR and GMV in medial prefrontal and striatal (NAcc and caudate) areas.

2. There will be a positive correlation between SP and GMV in the amygdala and the hippocampus.

3. There will be a stronger rsFC between the VTA, NAcc, ACC and vmPFC in high SR individuals.

4. There will be a stronger rsFC between the amygdala, hippocampus, and PAG in high SP individuals.

The proposed hypotheses have been tested through experimental studies, which are fully described in the next section. These studies correspond to published or submitted articles to international peer-reviewed journals in their original format.

\section{Experimental studies}

Study 1: Linking personality and brain anatomy: a structural MRI approach to Reinforcement Sensitivity Theory.

Study 2: Reward network connectivity "at rest" is associated with reward sensitivity in healthy adults: A resting-state fMRI study.

Study 3: The manifestation of individual differences in sensitivity to punishment during resting state is modulated by eye state. 
Study 1

Linking personality and brain anatomy: a structural MRI approach to Reinforcement Sensitivity Theory

Jesús Adrián-Ventura, Víctor Costumero, Maria Antònia Parcet, César Ávila

Social Cognitive and Affective Neuroscience

Soc Cogn Affect Neurosci. 2019 Mar 5; 14(3):329-338

doi: $\underline{10.1093 / \mathrm{scan} / \mathrm{nsz} 011}$ 


\begin{abstract}
Reinforcement Sensitivity Theory (RST) proposes a widely used taxonomy of human personality linked to individual differences at both behavioral and neuropsychological levels that describe a predisposition to psychopathology. However, the body of RST research was based on animal findings, and little is known about their anatomical correspondence in humans. Here we set out to investigate MRI structural correlates (i.e. voxel-based morphometry) of the main personality dimensions proposed by the RST in a group of 400 healthy young adults who completed the Sensitivity to Punishment and Sensitivity to Reward Questionnaire (SPSRQ). Sensitivity to punishment scores correlated positively with the gray matter volume in the amygdala, whereas, sensitivity to reward scores correlated negatively with the volume in the left lateral and medial prefrontal cortex. Moreover, a negative relationship was found between the striatal volume and the reward sensitivity trait, but only for male participants. The present results support the neuropsychological basis of the RST by linking punishment and reward sensitivity to anatomical differences in limbic and frontostriatal regions, respectively. These results are interpreted based on previous literature related to externalizing and internalizing disorders, and they highlight the possible role of SPSRQ as a measure of proneness to these disorders.
\end{abstract}

Keywords: personality; voxel-based morphometry; limbic system; frontostriatal circuit; psychopathological predisposition 


\section{Introduction}

The role of specific brain systems in personality development has long been hypothesized (i.e. Pavlov, 1941; Eysenck, 1960). One of the most influential models proposed in the past fifty years is Reinforcement Sensitivity Theory (RST; Gray, 1970; Gray, 1982; Gray and McNaughton, 2000; Pickering and Gray, 2001; Corr, 2008; McNaughton and Corr, 2008; Corr and McNaughton, 2012), which proposes a detailed neuropsychological description of the neural circuits underlying personality and psychopathological predisposition. The current version of the RST proposes three different systems responsible for taking control of behavior in the presence of emotional stimuli. The fight-flight-freeze system (FFFS) is neurally represented by an interconnected circuit comprising the amygdala (fear perception) and the medial hypothalamus and periaqueductal gray matter (behavioral response), and it mediates reactions to aversive stimuli, promoting defensive avoidance behavior. The behavioral inhibition system (BIS) has traditionally been related to the septo-hippocampal system, although in the last revision of the theory (see Gray and McNaughton, 2000; McNaughton and Corr, 2004), the amygdala was also included, and it responds specifically to goal conflict (i.e. approach-approach, approachavoidance or avoidance-avoidance) to initiate defensive approach behavior. Finally, the behavioral approach system (BAS) is dependent on the frontostriatal system, mainly the ventral [nucleus accumbens (NAcc)] and dorsal (caudate nucleus) striatum and its dopaminergic connections to the prefrontal lobe, and it mediates reactions to appetitive stimuli, promoting approach.

Although the description of the three neural systems should involve three different personality dimensions, the currently most accepted RST framework solution shows the existence of two general traits (Perkins et al., 2007). The first is punishment sensitivity, which depends on the combination of the FFFS and BIS systems in a global fear/anxiety dimension, and the second is reward sensitivity, which is associated with the BAS. These two dimensions could be assessed with specific measures such as the BIS/BAS scales, the SPSRQ, or the combination of the extraversion and neuroticism dimensions of the EPQ (Torrubia et al., 2008). Overall, the different punishment sensitivity measures typically show stronger correlations with each other (and with anxiety and fear measures), whereas the reward sensitivity measures only have moderate relationships with each other (Caseras et al., 2003).

Predictions of RST are very precise in the field of learning and conditioning. The theory postulates that individual differences in the activity of the FFFS/BIS and the BAS would be associated with: a) a different capacity to detect aversive and appetitive stimuli, respectively; and b) a different probability and intensity of emitting defensive and approach responses in the presence of these stimuli (see Ávila and Torrubia, 2008; Corr, 2008, for reviews). Different 
studies have confirmed that individuals with higher scores on measures of FFFS/BIS activity have better learning in the presence of aversive conditioned stimuli, and a greater probability of behavioral inhibition and better learning of the contingencies in aversive contexts. Similarly, individuals with higher scores on BAS measures have better learning and make more intense and probable approach responses in appetitive contexts.

RST has acquired great relevance in relating individual differences in the functioning of the FFFS, BIS, and BAS to the vulnerability to different psychopathologies. The RST formulation has related high FFFS activity to panic and phobic disorders (Barlow, 1988), and high BIS activity to generalized anxiety disorders (Maack et al., 2012). At the other extreme, evidence exists relating low BIS activity to psychopathy in adults (Fowles, 1980; Newman et al., 2005), and to the presence of callous/unemotional traits in children (Quay, 1988; Blair, 2003). In addition, some proposals relate high BAS activity to disinhibitory disorders such as attentiondeficit/hyperactivity disorder (ADHD; Newman and Wallace, 1993; Nigg, 2001; Mitchell and Nelson-Gray, 2006) or drug-addiction (Sher and Trull, 1994; Franken et al., 2006). At the opposite extreme, some reports have related depressive disorders to lower BAS activity (Pinto-Meza et al., 2006; Kimbrel et al., 2007). Overall, all these data link punishment sensitivity to the presence of internalizing disorders, and reward sensitivity to externalizing disorders (Bijttebier et al., 2009; Slobodskaya, 2016).

Although the detailed description of the brain circuits involved in these systems is one of the strongest points of the theory, this description is based on animal studies, and less research has been dedicated to verifying it in humans. The first attempt to test the neuropsychological basis of RST in the human brain was carried out by Barrós-Loscertales et al. (2006b), who reported a negative association between BAS activity and gray matter (GM) volume in the striatum and prefrontal cortex. Since then, only one study conducted in a large sample has provided new evidence relating BAS measures and brain anatomy (Holmes et al., 2016). In this study, a composite measure of novelty-seeking (NS) was associated with reduced cortical thickness in brain areas related to cognitive control, such as the dorsal anterior cingulate cortex, lateral prefrontal cortex, and supramarginal gyrus. Consistent with these anatomical studies, a DTI study that used the TCI of Cloninger showed that the two scales related to reward sensitivity were associated with different connectivity measures of the striatum (Lei et al., 2014). Specifically, the NS scale was related to stronger connectivity between the striatum and the hippocampus/amygdala, whereas the reward dependence scale was related to stronger connectivity between the striatum and different medial and lateral areas of the prefrontal cortex. On the other hand, Barrós-Loscertales et al. (2006a) first linked BIS activity to higher GM volume in the amygdala and the hippocampus. However, these results have only been partially replicated. Previous studies only found associations with BIS measures in the amygdala (Iidaka et al., 2006) 
or the hippocampus (Cherbuin et al., 2008; Levita et al., 2014), whereas other studies found a relationship with both structures (Holmes et al., 2012). Thus, these previous studies have produced different results, and so further research is required to address this controversy.

Thus, the aim of the current study was to investigate the brain regions associated with the personality dimensions depicted in the RST. Specifically, our objective was to extend our previous reports (Barrós-Loscertales et al., 2006a; Barrós-Loscertales et al., 2006b) in a larger sample consisting of males and females. Additionally, we also conducted further analyses in order to investigate possible differences by sex. Based on previous studies, we hypothesized that an overactive BAS would be associated with lower GM volume in prefrontal and striatal areas, whereas an overactive FFFS/BIS would be linked to greater GM volume in the amygdala and hippocampus. Because personality traits in their extreme forms are considered vulnerability factors for personality and mood disorders (Gray and McNaughton, 2000; Bijttebier et al., 2009; Corr and McNaughton, 2015), greater knowledge about the underlying neural correlates of personality should also contribute to better understanding these clinical conditions in unmedicated participants. Therefore, our reports would add new evidence to the RST model as a valid neuropsychological framework to study the proneness to psychopathological disorders in humans.

\section{Materials and methods}

\section{Participants}

Four hundred participants (233 males, 167 females; mean age 23.08, SD 5.32; range 1844 years; years of education 14.46, SD 2.22) were studied after giving their prior informed consent. All the participants were recruited from a community sample through local advertisements and word of mouth. Most of them were undergraduate students (94.75\%), given that our research group is integrated in a university campus. Participants had no history of neurological or psychiatric disorders, major medical illnesses, or traumatic brain injury with loss of consciousness. Additionally, all participants were right-handed, according to the Edinburgh Handedness Inventory (Oldfield, 1971; Bryden, 1977). The experiment was approved by the Ethical Committee of the University Jaume I (Spain).

\section{Personality measurement}

The Sensitivity to Punishment and Sensitivity to Reward Questionnaire (SPSRQ; Torrubia et al., 2001) was used to assess individual differences in personality. The SPSRQ 
provides two subscales; sensitivity to reward (SR) and sensitivity to punishment (SP), in order to evaluate the activity of the BAS and BIS/FFFS subsystems, respectively.

\section{MRI acquisition and voxel-based morphometry}

Images were all acquired with the same 1.5-T Siemens Avanto scanner (Erlangen, Germany). A high-resolution structural T1-weighted MPRAGE sequence was acquired (TE, 3.8 ms; TR, 2200 ms; flip angle, 15; matrix, 256 x 256 x 160 mm; voxel size, 1 mm3). Voxel-based morphometry (VBM) was performed with the VBM8 toolbox (version r445; http://dbm.neuro.uni-jena.de/vbm/) for the SPM8 package (version 6313; Wellcome Department of Imaging Neuroscience, London, UK). We performed the standard preprocessing procedure suggested in the VBM8 manual, which included: 1) segmentation of the images into gray matter, white matter, and cerebrospinal fluid; 2) registration to a standard template provided by the International Consortium of Brain Mapping (ICBM); 3) DARTEL normalization of the gray matter segments to the MNI template; and 4) modulation by nonlinear components derived from spatial normalization. After the preprocessing, a data quality check was carried out by analyzing the sample homogeneity using covariance. Ten participants showed a covariance at least 2 standard deviations below the mean; however, only two participants were identified as outliers (poor data quality) and excluded from the final sample. Finally, images were spatially smoothed using an 8-mm FWHM Gaussian kernel.

\section{Statistical analysis}

Voxel-wise regression analyses were performed by taking GM volume as the dependent variable and scores on the SR and SP scales as covariates of interest within the framework of the general linear model in SPM8. We also added age, sex, and years of education as nuisance covariates in order to control possible effects on brain volume. In addition, absolute threshold masking was set at .20 to ensure that we only selected GM voxels. Finally, our results at the cluster level were also corrected for non-stationary smoothing.

The statistical significance threshold for the whole-brain analysis was determined following recent recommendations by Chen et al. (2019). Therefore, we established a voxel-level primary threshold of $\mathrm{p}<.0005$ uncorrected, whereas we thresholded the obtained results at $\mathrm{p}<$ .025 FWE corrected at the cluster level. In addition, we also investigated focal voxel-based morphometry differences in small a priori regions of interest (ROIs). Based on the key regions involved in the BAS, BIS, and FFFS subsystems, these regions of interest were located in rewardrelated areas (NAcc and caudate) for the SR analyses, and in punishment-related areas (amygdala 
and hippocampus) for the SP analyses. All the ROIs were defined for each hemisphere using the atlas provided by Neuromorphometrics, Inc. (http://Neuromorphometrics.com/) under academic subscription. Furthermore, as our previous study found differences in the anterior hippocampus (Barrós-Loscertales et al., 2006a), and this region has previously been related to anxiety-related behaviors (Bannerman et al., 2004; Fanselow and Dong, 2010), we decided to include this area as a ROI for the SP analysis. For this purpose, the left and right hippocampus were traced manually on contiguous coronal slices in an MNI template following the guidelines of Watson et al. (1992) and Hasboun et al. (1996). This segmentation was carried out by an expert tracer using the MRIcron software. Moreover, because the amygdala and the anterior segment of the hippocampus are adjacent structures, we applied an exclusive mask (the amygdala ROI) to the anterior hippocampal ROI in order to remove all the possible voxels contained in the anterior hippocampus that could be overlapping voxels in the amygdala. All the ROIs included in the SR and SP analyses are shown in Figure 1. The modulated GM volumes (without smoothing) were obtained for each ROI via a MATLAB script (http://www0.cs.ucl.ac.uk/staff/g.ridgway/vbm/get_totals.m) and applying an absolute threshold masking of .20. After that, partial correlations (one-tailed based on a priori hypotheses) were performed using IBM SPSS Statistics 25 (Armonk, NY: IBM Corp.), including GM volumes and scores on the SR and SP scales as variables, controlling for age, sex, and years of education. Because we used four ROIs for the SR (left and right NAcc and caudate) and SP (left and right amygdala and anterior hippocampus) analyses, the statistical threshold for multiple comparisons $(\mathrm{p}<.05 \mathrm{FWE}$ ) was set at $\mathrm{p}<.0125$. Finally, because in our previous studies (Barrós-Loscertales et al., 2006a; Barrós-Loscertales et al., 2006b) the sample consisted only of male participants, we decided to conduct a new set of analyses in order to investigate possible sex differences. Thus, we carried out an omnibus model in SPM8 by calculating an interaction term with sex (including age and years of education as nuisance covariates). At the ROI level, as the analyses were performed in SPSS, sex differences were computed by analyzing the difference between the correlation coefficients using Fisher's z test.
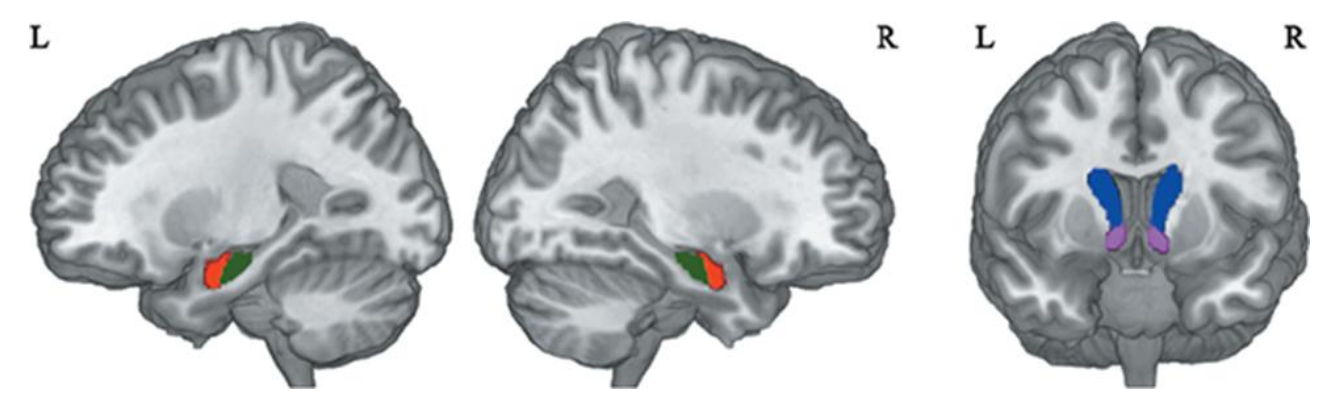

Fig. 1. ROIs included in the SR and SP analyses. Red: amygdala; Green: anterior hippocampus; Blue: caudate; Violet: nucleus accumbens. 


\section{Results}

\section{Personality scores}

Participants' mean score on the SR scale was 10.54 (SD 4.68), and the internal consistency was good (Cronbach's $\alpha=.81$ ). The mean score on the SP scale was 9.67 (SD 5.24), and its internal consistency was also high (Cronbach's $\alpha=.85$ ). Furthermore, SR and SP did not show any relationship with each other $(\mathrm{r}=-.05 ; \mathrm{p}=.371)$. Personality, demographic, and raw volume data for males and females are summarized in Table 1.

Table 1. Personality, demographic and volumetric data by sex.

\begin{tabular}{|c|c|c|c|c|c|}
\hline & $\begin{array}{l}\text { Whole sample } \\
\qquad(\mathrm{N}=400)\end{array}$ & Males (N=233) & Females (N=167) & $\begin{array}{c}t \text {-value } \\
\text { Males vs Females }\end{array}$ & $p$-value \\
\hline \multirow{2}{*}{ Age } & $23.08(5.32)$ & $24.54(6.07)$ & $21.05(3.04)$ & \multirow{2}{*}{7.56} & \multirow{2}{*}{$.000 * *$} \\
\hline & $18-44$ & $18-44$ & $18-42$ & & \\
\hline \multirow{2}{*}{ Years of education } & $14.46(2.22)$ & $14.30(2.41)$ & $14.69(1.91)$ & \multirow{2}{*}{-1.85} & \multirow{2}{*}{.066} \\
\hline & $8-20$ & $8-20$ & $8-19$ & & \\
\hline \multirow{2}{*}{ SR } & $10.54(4.68)$ & $11.73(4.80)$ & $8.88(3.98)$ & \multirow{2}{*}{6.49} & \multirow{2}{*}{$.000 * *$} \\
\hline & $1-23$ & $3-23$ & $1-20$ & & \\
\hline \multirow{2}{*}{ SP } & $9.67(5.24)$ & $8.70(5.01)$ & $11.02(5.27)$ & \multirow{2}{*}{-4.46} & \multirow{2}{*}{$.000 * *$} \\
\hline & $0-23$ & $0-22$ & $1-23$ & & \\
\hline \multirow{2}{*}{ Left NAcc } & $0.51(0.06)$ & $0.50(0.06)$ & $0.53(0.06)$ & \multirow{2}{*}{-5.39} & \multirow{2}{*}{$.000 * *$} \\
\hline & $0.28-0.71$ & $0.28-0.67$ & $0.39-0.71$ & & \\
\hline \multirow{2}{*}{ Right NAcc } & $0.48(0.06)$ & $0.46(0.05)$ & $0.50(0.05)$ & \multirow{2}{*}{-7.07} & \multirow{2}{*}{$.000 * *$} \\
\hline & $0.26-0.64$ & $0.26-0.58$ & $0.38-0.64$ & & \\
\hline \multirow{2}{*}{ Left Caudate } & $3.20(0.39)$ & $3.09(0.37)$ & $3.36(0.36)$ & \multirow{2}{*}{-7.51} & \multirow{2}{*}{$.000 * *$} \\
\hline & $1.86-4.27$ & $1.86-4.21$ & $2.43-4.27$ & & \\
\hline \multirow{2}{*}{ Right Caudate } & $3.04(0.40)$ & $2.93(0.38)$ & $3.20(0.36)$ & \multirow{2}{*}{-7.33} & \multirow{2}{*}{$.000 * *$} \\
\hline & $1.50-4.13$ & $1.50-3.91$ & $2.40-4.13$ & & \\
\hline \multirow{2}{*}{ Left Amygdala } & $1.04(0.09)$ & $1.03(0.08)$ & $1.05(0.10)$ & \multirow{2}{*}{-2.01} & \multirow{2}{*}{$.046^{*}$} \\
\hline & $0.79-1.33$ & $0.79-1.25$ & $0.81-1.33$ & & \\
\hline \multirow{2}{*}{ Right Amygdala } & $1.00(0.09)$ & $0.99(0.08)$ & $1.02(0.09)$ & \multirow{2}{*}{-3.56} & \multirow{2}{*}{$.000 * *$} \\
\hline & $0.78-1.27$ & $0.78-1.27$ & $0.81-1.23$ & & \\
\hline \multirow{2}{*}{ Left ant. hippocampus } & $1.13(0.10)$ & $1.12(0.09)$ & $1.14(0.10)$ & \multirow{2}{*}{-1.65} & \multirow{2}{*}{.099} \\
\hline & $0.83-1.45$ & $0.85-1.42$ & $0.83-1.45$ & & \\
\hline \multirow{2}{*}{ Right ant. hippocampus } & $1.29(0.11)$ & $1.28(0.11)$ & $1.31(0.11)$ & 267 & $008 * *$ \\
\hline & $1.00-1.64$ & $1.00-1.64$ & $1.06-1.59$ & -2.01 & .000 \\
\hline
\end{tabular}




\section{Sensitivity to Reward}

The voxel-wise GM analysis for the whole sample showed a negative correlation between SR scores and GM volume in frontal and temporal areas (see Table 2 for details). The most relevant reductions were located in the medial prefrontal cortex (from dorsomedial to ventromedial regions and also including the dorsal anterior cingulate cortex) and the left lateral prefrontal cortex, with a cluster extending from the middle to the inferior frontal cortex (see Figure 2A and Figure 3). No positive correlations were found between SR scores and GM volume. Moreover, the omnibus model did not reveal any significant differences by sex.

Table 2. Brain regions showing a negative correlation between SR scores and the voxel-wise GM volume.

\begin{tabular}{|c|c|c|c|c|c|}
\hline Region & Hemisphere & $\begin{array}{l}\text { Coordinates } \\
\text { MNI x, y, z }\end{array}$ & $t$-value & $k$-voxels & $p$-value \\
\hline Inferior frontal (pars opercularis) & $\mathrm{L}$ & $-56,8,21$ & 4.87 & 1731 & 0.0004 \\
\hline Middle frontal & $\mathrm{L}$ & $-30,45,28$ & 4.84 & & \\
\hline Inferior frontal (pars triangularis) & $\mathrm{L}$ & $-45,44,13$ & 4.43 & & \\
\hline Precentral & $\mathrm{L}$ & $-54,-3,30$ & 4.23 & & \\
\hline Inferior frontal (pars orbitalis) & $\mathrm{L}$ & $-47,20,-6$ & 3.86 & & \\
\hline Anterior cingulate & $\mathrm{L}$ & $-2,50,1$ & 4.37 & 1489 & 0.0008 \\
\hline Medial superior frontal & $\mathrm{L}$ & $-6,62,15$ & 4.17 & & \\
\hline Medial superior frontal & $\mathrm{R}$ & $3,57,15$ & 3.95 & & \\
\hline Middle temporal & $\mathrm{L}$ & $-51,-30,4$ & 4.45 & 851 & 0.008 \\
\hline Superior temporal & $\mathrm{L}$ & $-59,-15,6$ & 4.15 & & \\
\hline Insula & $\mathrm{L}$ & $-38,-16,-2$ & 3.65 & & \\
\hline
\end{tabular}

R: right; L: left; $p<.05$ FWE cluster-level corrected (two-tailed t-tests) 

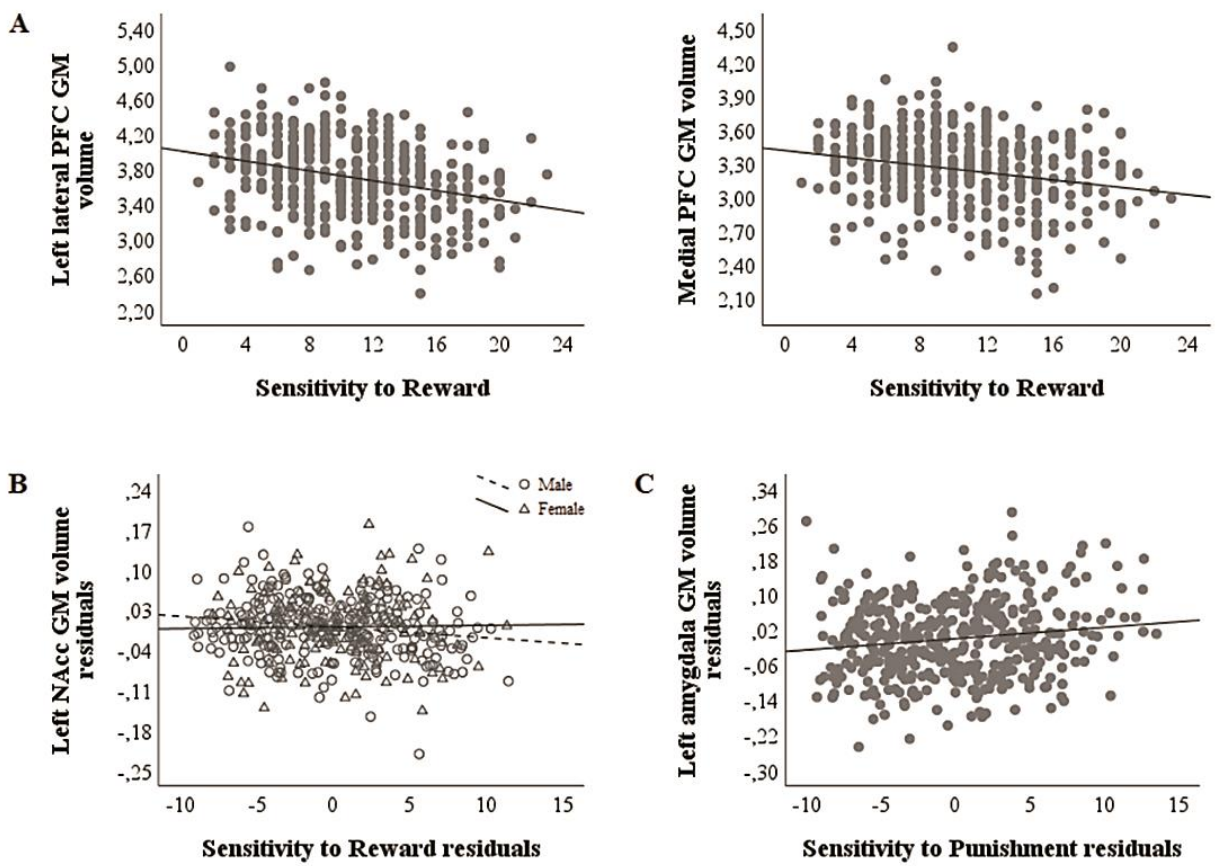

Fig. 2. A) Correlation between SR scores and GM volume in the left lateral and medial prefrontal clusters derived from the voxel-wise multiple regression analysis in the whole sample (controlling for age, sex, and years of education). B) Partial correlations for males and females showing a GM reduction in the left NAcc in males with high SR in comparison with females (after regressing out age and years of education). C) Partial correlation of the left amygdala GM volume with SP scores in the whole sample (after regressing out age, sex, and years of education). NAcc, nucleus accumbens; PFC, prefrontal cortex.

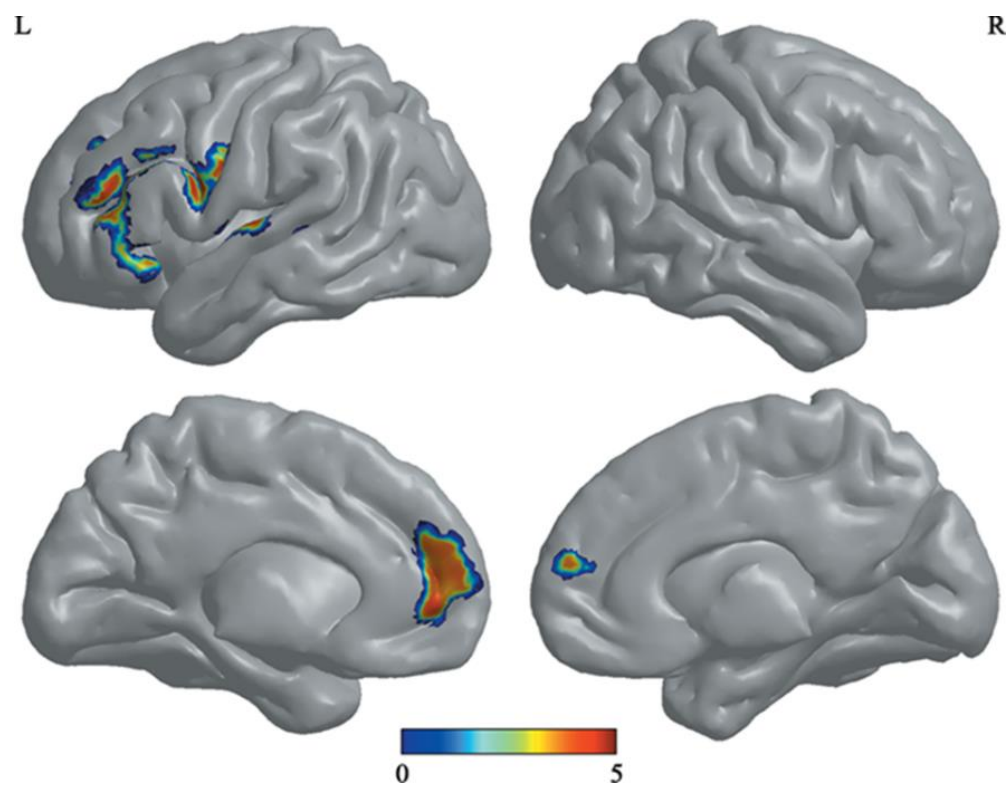

Fig. 3. Negative correlation between scores on the SR scale and GM volume in the left lateral and medial prefrontal cortex and superior temporal regions ( $p<.05$ FWE corrected, two-tailed). Bar color represents $t$ values. L, left; R, right. 
The partial correlations between SR scores and striatal ROI volumes appear on Table 3. Contrary to our hypothesis, the expected negative relationship between SR scores and striatum volume was only significant in males for the left NAcc and caudate volumes. Results of Fisher's $\mathrm{z}$ tests comparing the correlation coefficients between males and females reached significance only for the left NAcc $(z=-1.83 ; p=.034$; see Figure $2 B)$.

Table 3. Partial correlations (r) between a priori ROIs and scores on the SR scale (controlling for age, sex, and years of education).

\begin{tabular}{lccc}
\hline & Whole sample $(\mathbf{N}=\mathbf{4 0 0})$ & Males $(\mathbf{N}=\mathbf{2 3 3})$ & Females $(\mathbf{N}=\mathbf{1 6 7})$ \\
\hline Left NAcc & -.09 & $-.17^{*}$ & .02 \\
Right NAcc & -.03 & -.06 & .01 \\
Left Caudate & -.09 & $-.15^{*}$ & -.00 \\
Right Caudate & -.09 & -.13 & -.04 \\
\hline
\end{tabular}

One-tailed t-tests; $* p<.05$ FWE corrected.

\section{Sensitivity to Punishment}

The voxel-wise GM analyses for the whole sample did not show any significant negative or positive correlations with the SP scale. Similarly, the omnibus model did not reveal any significant differences by sex.

At the ROI level, we observed a significant positive correlation between the scores on the SP scale and the GM volume in the left amygdala $(\mathrm{r}=.15 ; \mathrm{p}=.002$, FWE corrected; see Figure 2C). Moreover, when analyzing the correlation coefficients in males and females, only the left amygdala showed a significant, positive association in males. However, Fisher's z test did not show any significant differences between males and females.

Table 4. Partial correlations (r) between a priori ROIs and scores on the SP scale (controlling for age, sex, and years of education).

\begin{tabular}{lccc}
\hline & Whole sample $(\mathbf{N}=\mathbf{4 0 0})$ & Males $(\mathbf{N}=\mathbf{2 3 3})$ & Females $(\mathbf{N}=\mathbf{1 6 7})$ \\
\hline Left Amygdala & $.15^{*}$ & $.15^{*}$ & .13 \\
Right Amygdala & .08 & .07 & .09 \\
Left ant. hippocampus & .09 & .11 & .05 \\
Right ant. hippocampus & .07 & .07 & .07 \\
\hline
\end{tabular}

One-tailed t-tests; $* \mathrm{p}<.05$ FWE corrected. 


\section{Discussion}

In the present study, we aimed to reveal the anatomical base underlying individual differences in the main personality dimensions depicted by the RST in a sample of 400 healthy participants studied with the same MRI scanner. Consistent with previous research, our results showed that scores on the sensitivity to punishment scale were positively associated with the GM volume in the left amygdala. Regarding sensitivity to reward, we found a direct negative relationship between reward sensitivity and the volume of the left lateral and medial prefrontal cortex. Furthermore, we have replicated our previous results relating this dimension to the striatum volume (Barrós-Loscertales et al., 2006b), but this result was not found in females. Thus, the main personality dimensions derived from the RST that measure vulnerability to the main psychopathological entities have been associated with differences in GM volume in target emotional brain areas. These results reinforce the usefulness of the SPSRQ for measuring individual differences in the neuropsychological systems depicted by the RST.

Our results are consistent with the RST and previous reports relating a greater GM volume in the amygdala to the scores on the SP scale from the SPSRQ (see Barrós-Loscertales et al., 2006a), on the Harm Avoidance scale (Iidaka et al., 2006), and on Neuroticism (Koelsch et al., 2013). Furthermore, in a study conducted in a large sample (Holmes et al., 2012), the authors reported a GM increase in the amygdala associated with scores on a customized scale based on behavioral inhibition and trait negative affect measures. Studies in healthy children have also demonstrated that inhibited or anxious personalities are linked to greater amygdala volume (Clauss et al., 2014; Qin et al., 2014). When studying pathological populations, enlargement of the amygdala has been reported in adults, children, and adolescents with Generalized Anxiety Disorder (De Bellis et al., 2000; Etkin et al., 2009; Schienle et al., 2011), and in adults with depression (Bremner et al., 2000; Tebartz van Elst et al., 2000; Lange and Irle, 2004; van Eijndhoven et al., 2009; Vassilopoulou et al., 2013), although results with depressive patients can vary depending on medication effects (see Hamilton et al., 2008). Moreover, the amygdala structure has also been associated with certain genetic alleles in panic disorder (Smoller et al., 2014). At the opposite pole, the presence of psychopathic or callous/unemotional traits in children and adult populations has been consistently associated with lower amygdala volume (Yang et al., 2009; Pardini et al., 2014; Vieira et al., 2015; Aghajani et al., 2016; Coccaro et al., 2016; Cohn et al., 2016), which has also been associated with conduct problems in youths (Rogers and De Brito, 2016). Among the different factors that contribute to modulating the amygdala volume, previous studies have reported the influence of genetic factors (Smoller et al., 2014) and early experiences with adverse situations (Howell et al., 2014). Regarding the hippocampal volume, contrary to our expectations, the expected positive association between SP scores and GM volume in the anterior hippocampal ROI did not reach significance. Although previous studies have found a significant 
relationship between the GM volume in the hippocampus and scores on BIS measures (BarrósLoscertales et al., 2006a; Cherbuin et al., 2008; Holmes et al., 2012; Levita et al., 2014), some methodological issues could account for these differences. One of them could be associated with the sample, as these previous studies were carried out with reduced sample sizes (BarrósLoscertales et al., 2006a; Levita et al., 2014) or focused on a specific age range (middle-aged adults, who differ significantly from our sample, see Cherbuin et al., 2008). Another methodological issue would be associated with the segmentation methods because, in contrast to our study, manual (Cherbuin et al., 2008) and automated (via FreeSurfer, Holmes et al., 2012; Levita et al., 2014) segmentations were applied to delimitate the amygdala and the hippocampus. Additionally, the study by Holmes et al. (2012) was carried out by taking a composite score of negative affect, which impedes the comparison with a direct single-scale score (i.e. SP). In addition, in the study by DeYoung et al. (2010), the authors did not find a positive association between the hippocampus and Neuroticism. In fact, they reported an inverse correlation between Neuroticism scores and hippocampal GM volume. Thus, further studies are needed to address the discrepancies about the involvement of the hippocampus and its anatomical correlates in behavioral inhibition. All in all, our results are in line with the last update of the RST and previous studies on the involvement of the amygdala in both fear and anxiety (McNaughton and Corr, 2004) and internalizing disorders.

The results have also shown a link between SR scores and reduced cortical volume in the lateral prefrontal cortex. The observed relationship was consistent with a recent study conducted in a large sample using impulsivity and sensation-seeking measures (Holmes et al., 2016), and with other studies that used the same self-reported measure of impulsivity (i.e. the Barratt Impulsivity Scale; Matsuo et al., 2009; Schilling et al., 2012; Cho et al., 2013). In addition, some studies have found that participants with lower GM volume in this region show a preference for immediate rewards on a delay-discounting measure (Bjork et al., 2009; Mohammadi et al., 2016; Wang et al., 2016; Wang et al., 2017). Therefore, less GM volume and cortical thickness in the lateral prefrontal areas may predispose these people to worse cognitive control, which may determine a different probability of involvement in risky, goal-directed activities such as substance use (Holmes et al., 2016). Consistent with previous studies on impulsivity (Cho et al., 2013; Korponay et al., 2017), SR also correlated negatively with the volume in the medial prefrontal cortex, a brain area crucial for evaluating rewarding stimuli (Hayes et al., 2014; Hiser and Koenigs, 2018), especially in subjective value-based decision-making (Bartra et al., 2013; Clithero and Rangel, 2014; Acikalin et al., 2017). Moreover, this region has traditionally been established as a key region for emotion regulation [via top-down regulatory control of limbic areas (Ridderinkhof et al., 2004; Etkin et al., 2011; Kim et al., 2011)]. Hence, the pattern of GM associated with the reward sensitivity dimension found in our study suggests that individuals with 
an overactive BAS have worse emotional control of reward experiences, facilitating goal-directed behavior in risky situations or even engagement in more violent behaviors (Coccaro et al., 2018). Furthermore, these reductions in prefrontal areas have also been related to externalizing disorders, principally substance use disorders (see Yang et al., 2016, for a meta-analysis on alcohol; Kaag et al., 2018) or even behavioral addictions, such as Internet gambling disorder (Yao et al., 2017).

Contrary to our expectations, the association between striatal volume and SR scores was modulated by sex. The negative correlation between the volume of the NAcc and SR scores was observed in males, but not in females. This result verified our previous results in males (BarrósLoscertales et al., 2006b) and clarified that this relationship does not extend to females. Interestingly, this differential pattern in males vs females in striatal areas was discussed in a recent paper (Caravaggio et al., 2017) in which impulsivity scores, measured by the TCI of Cloninger, correlated negatively with the striatal volume in males but not in females. Furthermore, a recent prospective study reported a correlation between striatal volume and Facebook use, but noting that effect sizes were clearly higher in males than females (Montag et al., 2017). Regarding its clinical impact, smaller striatal volumes have been reported in a number of externalizing disorders associated with an overactive BAS, with a higher prevalence in males. Some of these disorders included cocaine addiction (Barrós-Loscertales et al., 2011; Moreno-López et al., 2012), alcoholism (Makris et al., 2008; Yang et al., 2016), nicotine addiction (Das et al., 2012), pornography consumption (Kühn and Gallinat, 2014), and ADHD (see Hoogman et al., 2017, for a mega-analysis).

The explanation of this sex effect is less clear. Animal research has linked differences in the dopaminergic function to sex-specific transcriptome profiles within the NAcc (Hodes et al., 2015), with females being more susceptible to stress cues (i.e. less prone to reward-related behaviors, in comparison with males). However, these different transcriptome profiles could be associated with complex genetic and/or hormonal interactions (Becker and Chartoff, 2019). In humans, the sex effect on the striatal volume has also been associated with different functioning of the striatal dopaminergic system (i.e. dopamine receptor availability; see Caravaggio et al., 2017). Moreover, the volume of the NAcc has been associated with changes in testosterone levels during adolescence in males (Wierenga et al., 2018), coinciding in time with the maximum activity during processing of reward stimuli (Braams et al., 2015). Thus, although more research is needed, genetic, hormonal, and developmental factors may contribute to sex effects on the relationship between striatum volume and reward sensitivity. In addition, we should also take into account that these differences could be influenced by sex-specific behaviors in variables related to health and lifestyle; thus, future studies should also address the neuropsychological impact of these variables. In sum, we found an association between SR scores and the GM volume in 
frontostriatal areas, pointing to the link between individual differences in personality traits and brain structures found to be relevant in the development of externalizing disorders.

Since 1970, RST has developed into a sophisticated model of emotion, motivation, personality, psychopathology, and neuroscience. Overall, our study adds new evidence to previous reports linking brain structure and personality traits, revealing that the amygdala, the striatum (especially in males), and the prefrontal cortex are key regions associated with personality development. These results strengthen the role of the SPSRQ in measuring the main dimensions of the RST, and the role of the RST as a good, validated framework with which to investigate the predisposition to psychopathology.

Finally, our study has some limitations. Although we reported significant brain correlates with RST in a large sample, surface-based metrics could account for more specific effects than VBM. Indeed, VBM is a product of cortical thickness and surface area; however, the two measures have been shown to be genetically dissociated (Panizzon et al., 2009). Thus, future research should address this issue in order to better comprehend the biological underpinnings of personality traits. Another limitation would be related to the SPSRQ because this questionnaire provides a unique, combined measure (the SP scale) of the BIS and FFFS. Although both systems are strongly associated with the punishment sensitivity dimension and share neuroanatomical structures (i.e. the amygdala), they are described as separate neuropsychological systems. Nonetheless, no agreement exists about how the BIS and FFFS can be psychometrically isolated. Hence, future studies are required to address this question because the factorization of the SP scale would provide more accurate measures of the BIS and FFFS.

\section{Funding}

This work was supported by grants from Generalitat Valenciana (PROMETEO/2017/109) and Ministerio de Economía y Competitividad (PSI2016-78805-R) to C.A. Also, this work was supported by a pre-doctoral graduate program grant (National FPU to J.A.-V.) and a post-doctoral graduate program grant (Juan de la Cierva to V.C.).

\section{References}

Acikalin, M.Y., Gorgolewski, K.J., Poldrack, R.A. (2017). A coordinate-based meta-analysis of overlaps in regional specialization and functional connectivity across subjective value and default mode networks. Frontiers in Neuroscience, 11, 1. 
Aghajani, M., Klapwijk, E.T., van der Wee, N.J., et al. (2016). Disorganized amygdala networks in conduct-disordered juvenile offenders with callous-unemotional traits. Biological Psychiatry, $82,283-93$.

Ávila, C., Torrubia, R. (2008). Performance and conditioning studies. In: Corr, P.J., editor. The Reinforcement Sensitivity Theory of Personality, New York: Cambridge University Press, 228260.

Bannerman, D.M., Rawlins, J.N.P., McHugh, S.B., et al. (2004). Regional dissociations within the hippocampus - memory and anxiety. Neuroscience and Biobehavioral Reviews, 28, 273-283. Barlow, D.H. (1988). Anxiety and its Disorders: The Nature and Treatment of Anxiety Disorder, New York: Guilford.

Barrós-Loscertales, A., Garavan, H., Bustamante, J.C., et al. (2011). Reduced striatal volume in cocaine-dependent patients. NeuroImage, 56, 1021-1026.

Barrós-Loscertales, A., Meseguer, V., Sanjuán, A., et al. (2006a). Behavioral Inhibition System activity is associated with increased amygdala and hippocampal gray matter volume: a voxelbased morphometry study. NeuroImage, 33, 1011-1015.

Barrós-Loscertales, A., Meseguer, V., Sanjuán, A., et al. (2006b). Striatum gray matter reduction in males with an overactive behavioral activation system. European Journal of Neuroscience, 24, 2071-2074.

Bartra, O., McGuire, J.T., Kable, J.W. (2013). The valuation system: a coordinate-based metaanalysis of BOLD fMRI experiments examining neural correlates of subjective value. NeuroImage, 76, 412-427.

Becker, J.B., Chartoff, E. (2019). Sex differences in neural mechanisms mediating reward and addiction. Neuropsychopharmacology, 44, 166-183.

Bijttebier, P., Beck, I., Claes, L., et al. (2009). Gray's Reinforcement Sensitivity Theory as a framework for research on personality-psychopathology associations. Clinical Psychology Review, 29, 421-430.

Bjork, J.M., Momenan, R., Hommer, D.W. (2009). Delay discounting correlates with proportional lateral frontal cortex volumes. Biological Psychiatry, 65, 710-713.

Blair, C. (2003). Behavioral inhibition and behavioral activation in young children: relations with self-regulation and adaptation to preschool in children attending Head Start. Developmental Psychobiology, 42, 301-311. 
Braams, B.R., van Duijvenvoorde, A.C.K., Peper, J.S., et al. (2015). Longitudinal changes in adolescent risk-taking: a comprehensive study of neural responses to rewards, pubertal development, and risk-taking behavior. Journal of Neuroscience, 35, 7226-38.

Bremner, J.D., Narayan, M., Anderson, E.R., et al. (2000). Hippocampal volume reduction in major depression. American Journal of Psychiatry, 157, 115-118.

Bryden, M.P. (1977). Measuring handedness with questionnaires. Neuropsychologia, 15, 617624.

Caravaggio, F., Plitman, E., Chung, J.K., et al. (2017). Trait impulsiveness is related to smaller post-commissural putamen volumes in males but not females. European Journal of Neuroscience, 46, 2253-2264.

Caseras, X., Àvila, C., Torrubia, R. (2003). The measurement of individual differences in Behavioural Inhibition and Behavioural Activation Systems: a comparison of personality scales. Personality and Individual Differences, 34, 999-1013.

Chen, G., Cox, R.W., Glen, D.R., et al. (2019). A tail of two sides: artificially doubled false positive rates in neuroimaging due to the sidedness choice with t-tests. Human Brain Mapping, 40, 1037-1043.

Cherbuin, N., Windsor, T.D., Anstey, K.J., et al. (2008). Hippocampal volume is positively associated with behavioural inhibition (BIS) in a large community-based sample of mid-life adults: the PATH through life study. Social Cognitive and Affective Neuroscience, 3, 262-269.

Cho, S.S., Pellecchia, G., Aminian, K., et al. (2013). Morphometric correlation of impulsivity in medial prefrontal cortex. Brain Topography, 26, 479-487.

Clauss, J.A., Seay, A.L., VanDerKlok, R.M., et al. (2014). Structural and functional bases of inhibited temperament. Social Cognitive and Affective Neuroscience, 9, 2049-2058.

Clithero, J.A., Rangel, A. (2014). Informatic parcellation of the network involved in the computation of subjective value. Social Cognitive and Affective Neuroscience, 9, 1289-1302.

Coccaro, E.F., Cremers, H., Fanning, J., et al. (2018). Reduced frontal grey matter, life history of aggression, and underlying genetic influence. Psychiatry Research: Neuroimaging, 271, 126134.

Coccaro, E.F., Fitzgerald, D.A., Lee, R., et al. (2016). Frontolimbic morphometric abnormalities in intermittent explosive disorder and aggression. Biological Psychiatry: Cognitive Neuroscience and Neuroimaging, 1, 32-38. 
Cohn, M.D., Viding, E., McCrory, E., et al. (2016). Regional grey matter volume and concentration in at-risk adolescents: untangling associations with callous-unemotional traits and conduct disorder symptoms. Psychiatry Research: Neuroimaging, 254, 180-187.

Corr, P.J. (2008). Reinforcement Sensitivity Theory (RST): introduction. In: Corr, P.J., editor. The Reinforcement Sensitivity Theory of Personality, Cambridge: Cambridge University Press, $1-43$.

Corr, P.J., McNaughton, N. (2015). Neural mechanisms of low trait anxiety and risk for externalizing behavior. In: Beauchaine, P.J., Hinshaw, S.P., editors. The Oxford Handbook of Externalizing Spectrum Disorders, New York: Oxford University Press, 220-238.

Corr, P.J., McNaughton, N. (2012). Neuroscience and approach/avoidance personality traits: a two stage (valuation-motivation) approach. Neuroscience and Biobehavioral Reviews, 36, 2339 2354.

De Bellis, M.D., Casey, B.J., Dahl, R.E., et al. (2000). A pilot study of amygdala volumes in pediatric generalized anxiety disorder. Biological Psychiatry, 48, 51-57.

Das, D., Cherbuin, N., Anstey, K.J., et al. (2012). Lifetime cigarette smoking is associated with striatal volume measures. Addiction Biology, 17, 817-825.

DeYoung, C.G., Hirsh, J.B., Shane, M.S., et al. (2010). Testing predictions from personality neuroscience. Brain structure and the big five. Psychological Science, 21, 820-828.

Etkin, A., Egner, T., Kalisch, R. (2011). Emotional processing in anterior cingulate and medial prefrontal cortex. Trends in Cognitive Sciences, 15, 85-93.

Etkin, A., Prater, K.E., Schatzberg, A.F., et al. (2009). Disrupted amygdalar subregion functional connectivity and evidence of a compensatory network in generalized anxiety disorder. Archives of General Psychiatry, 66, 1361-1372.

Eysenck, H.J. (1960). Experiments in Personality, London: Routledge \& Kegan Paul.

Fanselow, M.S., Dong, H.-W. (2010). Are the dorsal and ventral hippocampus functionally distinct structures? Neuron, 65, 7-19.

Fowles, D.C. (1980). The three arousal model: implications of Gray's two-factor learning theory for heart rate, electrodermal activity, and psychopathy. Psychophysiology, 17, 87-104.

Franken, I.H.A., Muris, P., Georgieva, I. (2006). Gray's model of personality and addiction. Addictive Behaviors, 31, 399-403. 
Gray, J.A. (1982). The Neuropsychology of Anxiety: An Enquiry Into the Functions of the SeptoHippocampal System, Oxford: Oxford University Press.

Gray, J.A. (1970). The psychophysiological basis of introversion-extraversion. Behaviour Research and Therapy, 8, 249-266.

Gray, J.A., McNaughton, N.J. (2000). The Neuropsychology of Anxiety: An Inquiry Into the Functions of the Septo-Hippocampal System, 2nd edn, Oxford: Oxford University Press.

Hamilton, J.P., Siemer, M., Gotlib, I.H. (2008). Amygdala volume in major depressive disorder: a meta-analysis of magnetic resonance imaging studies. Molecular Psychiatry, 13, 993-1000.

Hasboun, D., Chantôme, M., Zouaoui, A., et al. (1996). MR determination of hippocampal volume: comparison of three methods. AJNR. American Journal of Neuroradiology, 17, 10911098.

Hayes, D.J., Duncan, N.W., Xu, J., et al. (2014). A comparison of neural responses to appetitive and aversive stimuli in humans and other mammals. Neuroscience and Biobehavioral Reviews, 45, 350-368.

Hiser, J., Koenigs, M. (2018). The multifaceted role of the ventromedial prefrontal cortex in emotion, decision making, social cognition, and psychopathology. Biological Psychiatry, 83, 638-647.

Hodes, G.E., Pfau, M.L., Purushothaman, I., et al. (2015). Sex differences in nucleus accumbens transcriptome profiles associated with susceptibility versus resilience to subchronic variable stress. Journal of Neuroscience, 35, 16362-16376.

Holmes, A.J., Hollinshead, M.O., Roffman, J.L., et al. (2016). Individual differences in cognitive control circuit anatomy link sensation seeking, impulsivity, and substance use. Journal of Neuroscience, 36, 4038-4049.

Holmes, A.J., Lee, P.H., Hollinshead, M.O., et al. (2012). Individual differences in amygdalamedial prefrontal anatomy link negative affect, impaired social functioning, and polygenic depression risk. Journal of Neuroscience, 32, 18087-18100.

Hoogman, M., Bralten, J., Hibar, D.P., et al. (2017). Subcortical brain volume differences in participants with attention deficit hyperactivity disorder in children and adults: a cross-sectional mega-analysis. Lancet Psychiatry, 4, 310-319.

Howell, B.R., Grand, A.P., McCormack, K.M., et al. (2014). Early adverse experience increases emotional reactivity in juvenile rhesus macaques: relation to amygdala volume. Developmental Psychobiology, 56, 1735-1746. 
Iidaka, T., Matsumoto, A., Ozaki, N., et al. (2006). Volume of left amygdala subregion predicted temperamental trait of harm avoidance in female young subjects. A voxel-based morphometry study. Brain Research, 1125, 85-93.

Kaag, A.M., Schulte, M.H.J., Jansen, J.M., et al. (2018). The relation between gray matter volume and the use of alcohol, tobacco, cocaine and cannabis in male polysubstance users. Drug and Alcohol Dependence, 187, 186-194.

Kim, M.J., Loucks, R.A., Palmer, A.L., et al. (2011). The structural and functional connectivity of the amygdala: from normal emotion to pathological anxiety. Behavioural Brain Research, 223 , 403-410.

Kimbrel, N.A., Nelson-Gray, R.O., Mitchell, J.T. (2007). Reinforcement sensitivity and maternal style as predictors of psychopathology. Personality and Individual Differences, 42, 1139-1149.

Koelsch, S., Skouras, S., Jentschke, S., et al. (2013). Neural correlates of emotional personality: a structural and functional magnetic resonance imaging study. PLoS One, 8, e77196.

Korponay, C., Dentico, D., Kral, T., et al. (2017). Neurobiological correlates of impulsivity in healthy adults: lower prefrontal gray matter volume and spontaneous eye-blink rate but greater resting-state functional connectivity in basal ganglia-thalamo-cortical circuitry. NeuroImage, $157,288-296$.

Kühn, S., Gallinat, J. (2014). Brain structure and functional connectivity associated with pornography consumption. JAMA Psychiatry, 71, 827.

Lange, C., Irle, E. (2004). Enlarged amygdala volume and reduced hippocampal volume in young women with major depression. Psychological Medicine, 34, 1059-1064.

Lei, X., Chen, C., Xue, F., et al. (2014). Fiber connectivity between the striatum and cortical and subcortical regions is associated with temperaments in Chinese males. NeuroImage, 89, 226-234.

Levita, L., Bois, C., Healey, A., et al. (2014). The Behavioural Inhibition System, anxiety and hippocampal volume in a non-clinical population. Biology of Mood \& Anxiety Disorders, 4, 4.

Maack, D.J., Tull, M.T., Gratz, K.L. (2012). Examining the incremental contribution of behavioral inhibition to generalized anxiety disorder relative to other Axis I disorders and cognitive-emotional vulnerabilities. Journal of Anxiety Disorders, 26, 689-695.

Makris, N., Oscar-Berman, M., Jaffin, S.K., et al. (2008). Decreased volume of the brain reward system in alcoholism. Biological Psychiatry, 64, 192-202.

Matsuo, K., Nicoletti, M., Nemoto, K., et al. (2009). A voxel-based morphometry study of frontal gray matter correlates of impulsivity. Human Brain Mapping, 30, 1188-1195. 
McNaughton, N., Corr, P. (2008). The neuropsychology of fear and anxiety: a foundation for Reinforcement Sensitivity Theory. In: Corr, P.J., editor. The Reinforcement Sensitivity Theory of Personality, Cambridge: Cambridge University Press, 44-94.

McNaughton, N., Corr, P.J. (2004). A two-dimensional neuropsychology of defense: fear/anxiety and defensive distance. Neuroscience and Biobehavioral Reviews, 28, 285-305.

Mitchell, J.T., Nelson-Gray, R.O. (2006). Attention-Deficit/Hyperactivity Disorder symptoms in adults: relationship to Gray's Behavioral Approach System. Personality and Individual Differences, 40, 749-760.

Mohammadi, B., Hammer, A., Miedl, S.F., et al. (2016). Intertemporal choice behavior is constrained by brain structure in healthy participants and pathological gamblers. Brain Structure and Function, 221, 3157-3170.

Montag, C., Markowetz, A., Blaszkiewicz, K., et al. (2017). Facebook usage on smartphones and gray matter volume of the nucleus accumbens. Behavioural Brain Research, 329, 221-228.

Moreno-López, L., Catena, A., Fernández-Serrano, M.J., et al. (2012). Trait impulsivity and prefrontal gray matter reductions in cocaine dependent individuals. Drug and Alcohol Dependence, 125, 208-214.

Newman, J.P., MacCoon, D.G., Vaughn, L.J., et al. (2005). Validating a distinction between primary and secondary psychopathy with measures of Gray's BIS and BAS constructs. Journal of abnormal psychology, 114, 319-323.

Newman, J.P., Wallace, J.F. (1993). Diverse pathways to deficient self-regulation: implications for disinhibitory psychopathology in children. Clinical Psychology Review, 13, 699-720.

Nigg, J.T. (2001). Is ADHD a disinhibitory disorder? Psychological bulletin, 127, 571-598.

Oldfield, R.C. (1971). The assessment and analysis of handedness: the Edinburgh inventory. Neuropsychologia, 9, 97-113.

Panizzon, M.S., Fennema-Notestine, C., Eyler, L.T., et al. (2009). Distinct genetic influences on cortical surface area and cortical thickness. Cerebral Cortex, 19, 2728-2735.

Pardini, D.A., Raine, A., Erickson, K., et al. (2014). Lower amygdala volume in men is associated with childhood aggression, early psychopathic traits, and future violence. Biological Psychiatry, $75,73-80$.

Pavlov, I.P. (1941). Lectures on Conditioned Reflexes. Volume 2: Conditioned Reflexes and Psychiatry, London: Lawrence \& Wishart. 
Perkins, A.M., Kemp, S.E., Corr, P.J. (2007). Fear and anxiety as separable emotions: an investigation of the revised reinforcement sensitivity theory of personality. Emotion, 7, 252-261. Pickering, A.D., Gray, J.A. (2001). Dopamine, appetitive reinforcement, and the neuropsychology of human learning: an individual differences approach. In: Elias, A., Angleitner, A., editors. Advances in Individual Differences Research, Lengerich: PABST Science Publishers, $113-149$.

Pinto-Meza, A., Caseras, X., Soler, J., et al. (2006). Behavioural Inhibition and Behavioural Activation Systems in current and recovered major depression participants. Personality and Individual Differences, 40, 215-226.

Qin, S., Young, C.B., Duan, X., et al. (2014). Amygdala subregional structure and intrinsic functional connectivity predicts individual differences in anxiety during early childhood. Biological Psychiatry, 75, 892-900.

Quay, H.C. (1988). The behavioral reward and inhibition systems in child behavior disorder. In: Bloomingdale, L.M., editor. Attention Deficit Disorder. Volume 3: New Research in Attention, Treatment, and Psychopharmacology, New York: Pergamon, 176-186.

Ridderinkhof, K.R., Ullsperger, M., Crone, E.A., et al. (2004). The role of the medial frontal cortex in cognitive control. Science, 306, 443-447.

Rogers, J.C., De Brito, S.A. (2016). Cortical and subcortical gray matter volume in youths with conduct problems. JAMA Psychiatry, 73, 64.

Schienle, A., Ebner, F., Schäfer, A. (2011). Localized gray matter volume abnormalities in generalized anxiety disorder. European Archives of Psychiatry and Clinical Neuroscience, 261, 303-307.

Schilling, C., Kühn, S., Romanowski, A., et al. (2012). Cortical thickness correlates with impulsiveness in healthy adults. NeuroImage, 59, 824-830.

Sher, K.J., Trull, T.J. (1994). Personality and disinhibitory psychopathology: alcoholism and antisocial personality disorder. Journal of Abnormal Psychology, 103, 92-102.

Slobodskaya, H.R. (2016). The contribution of reinforcement sensitivity to the personalitypsychopathology hierarchical structure in childhood and adolescence. Journal of Abnormal Psychology, 125, 1067-1078.

Smoller, J.W., Gallagher, P.J., Duncan, L.E., et al. (2014). The human ortholog of acid-sensing ion channel gene ASIC1a is associated with panic disorder and amygdala structure and function. Biological Psychiatry, 76, 902-910. 
Tebartz van Elst, L., Woermann, F., Lemieux, L., et al. (2000). Increased amygdala volumes in female and depressed humans. A quantitative magnetic resonance imaging study. Neuroscience Letters, 281, 103-106.

Torrubia, R., Avila, C., Caseras, X. (2008). Reinforcement sensitivity scales. In: Corr, P.J., editor. The Reinforcement Sensitivity Theory of Personality, New York: Cambridge University Press, $188-226$.

Torrubia, R., Ávila, C., Moltó, J., et al. (2001). The Sensitivity to Punishment and Sensitivity to Reward Questionnaire (SPSRQ) as a measure of Gray's anxiety and impulsivity dimensions. Personality and Individual Differences, 31, 837-862.

van Eijndhoven, P., van Wingen, G., van Oijen, K., et al. (2009). Amygdala volume marks the acute state in the early course of depression. Biological Psychiatry, 65, 812-18.

Vassilopoulou, K., Papathanasiou, M., Michopoulos, I., et al. (2013). A magnetic resonance imaging study of hippocampal, amygdala and subgenual prefrontal cortex volumes in major depression subtypes: melancholic versus psychotic depression. Journal of Affective Disorders, 146, 197-204.

Vieira, J.B., Ferreira-Santos, F., Almeida, P.R., et al. (2015). Psychopathic traits are associated with cortical and subcortical volume alterations in healthy individuals. Social Cognitive and Affective Neuroscience, 10, 1693-1704.

Wang, Q., Chen, C., Cai, Y., et al. (2016). Dissociated neural substrates underlying impulsive choice and impulsive action. NeuroImage, 134, 540-549.

Wang, S., Kong, F., Zhou, M., et al. (2017). Brain structure linking delay discounting and academic performance. Human Brain Mapping, 38, 3917-3926.

Watson, C., Andermann, F., Gloor, P., et al. (1992). Anatomic basis of amygdaloid and hippocampal volume measurement by magnetic resonance imaging. Neurology, 42, 1743-1750.

Wierenga, L.M., Bos, M.G.N., Schreuders, E., et al. (2018). Unraveling age, puberty and testosterone effects on subcortical brain development across adolescence. Psychoneuroendocrinology, 91, 105-114.

Yang, X., Tian, F., Zhang, H., et al. (2016). Cortical and subcortical gray matter shrinkage in alcohol-use disorders: a voxel-based meta-analysis. Neuroscience and Biobehavioral Reviews, $66,92-103$.

Yang, Y., Raine, A., Narr, K.L., et al. (2009). Localization of deformations within the amygdala in individuals with psychopathy. Archives of General Psychiatry, 66, 986-994. 
Yao, Y.-W., Liu, L., Ma, S.-S., et al. (2017). Functional and structural neural alterations in Internet gaming disorder: a systematic review and meta-analysis. Neuroscience and Biobehavioral Reviews, 83, 313-324. 
Study 2

Reward network connectivity "at rest” is associated with reward sensitivity in healthy adults: A resting-state fMRI study

Jesús Adrián-Ventura, Víctor Costumero, Maria Antònia Parcet, César Ávila

Cognitive, Affective, \& Behavioral Neuroscience

Cogn Affect Behav Neurosci. 2019 Jun; 19(3):726-736

doi: $\underline{10.3758 / \mathrm{s} 13415-019-00688-1}$ 


\begin{abstract}
The behavioral approach system (BAS), based on reinforcement sensitivity theory (RST), is a neurobehavioral system responsible for detecting and promoting motivated behaviors towards appetitive stimuli. Anatomically, the frontostriatal system has been proposed as the core of the BAS, mainly the ventral tegmental area and the ventral striatum and their dopaminergic connections with medial prefrontal structures. The RST also proposes the personality trait of reward sensitivity as a measurable construct of stable individual differences in BAS activity. However, the relationship between this trait and the brain connectivity "at rest" has been poorly studied, mainly because previous investigations have focused on studying brain activity under reward-related contingency paradigms. Here, we analyzed the influence of reward sensitivity on the resting-state functional connectivity (rs-FC) between BAS-related areas by correlating the BOLD time series with the scores on the Sensitivity to Reward (SR) scale in a sample of 89 healthy young adults. Rs-FC between ROIs were all significant. Results also revealed a positive association between SR scores and the rs-FC between the VTA and the ventromedial prefrontal cortex, and between the latter structure and the anterior cingulate cortex. These results suggest that reward sensitivity could be associated with different resting-state activity in the mesocortical pathway.
\end{abstract}

Keywords: personality, reward, functional connectivity, basal ganglia, prefrontal cortex 


\section{Introduction}

Reinforcement sensitivity theory (RST) describes a widely used taxonomy of personality at both behavioral and neuropsychological levels (Gray, 1982; Gray \& McNaughton, 2000). This model proposes different brain systems responsible for promoting motivated behaviors to approach or avoid stimuli with a positive or negative emotional valence. In particular, the behavioral approach system (BAS) is responsible for detecting and initiating behaviors towards appetitive stimuli. Anatomically, the BAS is associated with brain structures belonging to the mesolimbic and mesocortical dopaminergic pathways (Pickering \& Gray, 1999, 2001). The key region is the ventral tegmental area (VTA), which is connected to the ventral striatum (VS; mesolimbic tract) and the medial prefrontal cortex (mesocortical tract), including the anterior cingulate (ACC) and the ventromedial prefrontal cortex (vmPFC). All these areas define the reward network, that is, the main structures involved in processing and responding to reward stimuli (see Haber \& Knutson, 2010; Sescousse, Caldú, Segura, \& Dreher, 2013).

According to the BAS description in the RST model, this system is involved not only in managing rewarding stimuli, but also in determining stable individual differences in the detection and response to appetitive stimuli. In other words, the model proposes that the reward sensitivity trait is associated with stable differences in BAS functioning, determining the probability of perceiving stimuli as rewarding and actively approaching them once detected (Ávila \& Torrubia, 2008; Corr, 2004; Gray \& McNaughton, 2000). To measure these differences, some psychometric measures have been developed, such as the Sensitivity to Reward (SR) scale included in the Sensitivity to Punishment and Sensitivity to Reward Questionnaire (SPSRQ; Torrubia, Ávila, Moltó, \& Caseras, 2001) or the three BAS subscales included in the BIS/BAS questionnaire (Carver \& White, 1994). Psychometric studies have shown that these measures are related to other personality traits such as extraversion, impulsivity, or sensation seeking (Caseras, Àvila, \& Torrubia, 2003; Torrubia, Avila, \& Caseras, 2008). BAS measures have also been found to be a good measure of proneness or vulnerability to some psychiatric pathologies. Higher reward sensitivity has been related to the presence of ADHD symptoms (Mitchell \& Nelson-Gray, 2006), substance use and abuse (Boog et al., 2014; Franken, Muris, \& Georgieva, 2006; Knyazev, 2004; Pardo, Aguilar, Molinuevo, \& Torrubia, 2007; Yen et al., 2012), eating disorders (Glashouwer, Bloot, Veenstra, Franken, \& de Jong, 2014; Matton, Goossens, Braet, \& Vervaet, 2013; Matton, Goossens, Vervaet, \& Braet, 2015), psychopathy (Newman, MacCoon, Vaughn, \& Sadeh, 2005), and cluster B personality disorders (Bijttebier, Beck, Claes, \& Vandereycken, 2009; Pastor et al., 2007; Taylor, Reeves, James, \& Bobadilla, 2006). Moreover, lower reward sensitivity has been associated with depressive disorders (Kasch, Rottenberg, Arnow, \& Gotlib, 2002; Pinto-Meza et al., 2006). 
Behavioral studies have consistently demonstrated that individuals with higher scores on these scales show better learning (i.e. conditioning) in appetitive situations than low scorers (i.e. those with lower reward sensitivity) (see Corr, 2004 for a review). On tasks requiring decision making, individuals with higher reward sensitivity focus their attention on rewarding stimuli (Derryberry \& Reed, 1994), show faster responses to obtain rewards in the presence of possible aversive stimuli (Avila, 2001), prefer immediate rewards over delayed rewards (Avila, Parcet, Ortet, \& Ibáñez-Ribes, 1999), and perseverate in dominant responses for rewards (Avila, Moltó, \& Segarra, 1995; Avila \& Parcet, 2000; Newman, Widom, \& Nathan, 1985), compared to individuals with lower reward sensitivity. This entire cognitive system oriented toward detecting reward cues and making approach responses to them is manifested in different social behaviors. As a result, individuals with higher reward sensitivity are more impulsive on multiple choice exams (Ávila \& Torrubia, 2004), show more food cravings (Franken \& Muris, 2006), have more sexual experiences (Aluja, 2004), exhibit early-stage entrepreneurial action (Lerner, Hatak, \& Rauch, 2018), or initiate the use of drugs at a younger age (Pardo et al., 2007).

Behavioral studies have also shown that reward sensitivity determines differences on cognitive tasks with no specific reward contingencies. According to Aarts, van Holstein, \& Cools (2011), individual differences in appetitive motivation appear to have parallel effects to those of increases in striatal dopamine, that is, the enhancement of cognitive flexibility, which may come, however, at the expense of reduced cognitive focusing (i.e. greater distractibility). There are different behavioral and fMRI studies showing that reward sensitivity determines differences in cognitive processing (Avila, 1995; Avila et al., 2012; Avila, Barrós, Ortet, Parcet, \& Ibañez, 2003; Avila \& Parcet, 1997; Avila \& Parcet, 2002; Fuentes-Claramonte et al., 2015, 2016a, 2016b, Fuentes et al., 2014a, 2014b). All this evidence is compatible with the idea that BAS-related traits should explain individual differences in cognition.

RST is based on neuropsychological studies mainly conducted in animal research. The RST model predicts specific relationships between certain brain areas and the different personality dimensions. However, these proposals have been investigated less in the human brain. Previous morphometric studies have found associations between BAS scores and gray matter reductions in BAS-related areas, including the striatum, the anterior cingulate cortex, and prefrontal areas (Barrós-Loscertales et al., 2006; Holmes, Hollinshead, Roffman, Smoller, \& Buckner, 2016). In addition, some fMRI studies have also linked BAS activation to brain activity in these basic structures. Specifically, task-based studies with different kinds of rewards (i.e. food and erotic stimuli, monetary incentives, etc.) have associated higher reward sensitivity with greater activation in brain areas involved in the mesolimbic and mesocortical pathways. Thus, a number of studies have found this association in mesolimbic areas, including the midbrain and ventral striatal regions (Beaver et al., 2006; Carter, Macinnes, Huettel, \& Adcock, 2009; 
Costumero et al., 2016; Costumero et al., 2013a; Costumero et al., 2013b; Hahn et al., 2009; Mortensen, Lehn, Evensmoen, \& Håberg, 2015; Simon et al., 2010), whereas others have also reported this correlation with ventromedial prefrontal regions of the mesocortical pathway (Barrós-Loscertales et al., 2010; Beaver et al., 2006; Costumero et al., 2013b; Hahn et al., 2009; Locke \& Braver, 2008; Mortensen et al., 2015; Simon et al., 2010). Functional connectivity analysis revealed that BAS scores were also associated with relatively less connectivity between the VTA and vmPFC during the processing of high-incentive monetary cues (Costumero et al., 2013a). Thus, BAS scores in healthy participants correlated with greater activity in the brain areas involved in the mesolimbic and mesocortical pathways during the processing of reward cues. Importantly, other research has demonstrated that BAS scores determine different activity in mesolimbic and prefrontal areas during performance on executive tasks (Avila et al., 2012; Fuentes-Claramonte et al., 2016a, 2016b, 2015, Fuentes et al., 2014a, 2014b).

All this evidence suggests that reward sensitivity is related to a different general and stable functioning of reward-related areas involved in motivation and emotion: VTA, VS, ACC, and vmPFC. This variability in the functioning of these areas could be studied by measuring the functional connectivity (FC) between them using resting-state fMRI. As far as we know, only one previous study investigated the FC between striatal and cortical areas, including the prefrontal and cingulate cortex, based on a BAS measure (Angelides, Gupta, \& Vickery, 2017). In this article, the authors reported a positive correlation between BAS-fun seeking and the FC between the middle OFC and the putamen, and a negative correlation between BAS-drive and the rs-FC between the middle cingulate cortex and the caudate. However, this study did not specifically investigate BAS relevant structures such as the VS or the VTA.

The aim of this study was to investigate the relationship between the reward sensitivity trait, measured with the SR scale from the SPSRQ, and the FC among key structures of the brain reward system (i.e. the VTA, VS, ACC, and vmPFC) in a sample of healthy adults. Based on previous reports, we hypothesize that the activity between all these structures will be positively associated, and that individuals with higher reward sensitivity would show a stronger FC between all these regions.

\section{Methods}

\section{Participants}

One hundred and twenty-eight participants took part in this study (63 women; age: mean=22.5, $\mathrm{SD}=4.8$, range=18-37). All the participants were recruited from a community sample through local advertisements and word of mouth. Most of them were undergraduate students, 
given that our research group is integrated in a university campus. Following subject exclusion due to excessive head motion (see preprocessing section), the final sample for analyses included 89 participants (48 women; age: mean $=22.4, \mathrm{SD}=4.7$, range=18-37). All the participants were right-handed, according to the Edinburgh Handedness Inventory (Oldfield, 1971). No participant had a history of head injury with loss of consciousness, currently used psychoactive medications, or had ever been diagnosed with DSM-IV Axis I or II disorders or severe medical or neurological illnesses. Participants were informed of the nature of the research, and they provided written informed consent prior to their participation in the study. All the study procedures conformed to the Code of Ethics of the World Medical Association.

\section{Personality assessment}

All the participants completed the Sensitivity to Reward (SR) scale from the Sensitivity to Punishment and Sensitivity to Reward Questionnaire (Torrubia et al., 2001) as a measure of reward sensitivity. This questionnaire is a self-report measure with 48 dichotomous items that assess the reactivity and responsivity of the behavioral inhibition and activation systems described by Gray (1982; Gray \& McNaughton, 2000). As our interest was reward sensitivity, only the SR scale was included in the study. The SR scale is a self-reported measure consisting of 24 yes-no items related to one's habitual behavior. In this particular case, the questions measure the proneness to approach behaviors towards appetitive stimuli. Different studies have shown the SR scale's convergent validity with other measures, as well as the association with disinhibitory and depressive pathologies (Torrubia et al., 2008). The mean score on the SR scale was 10.2 (SD = 4.7 ; range $=1-21$ ).

\section{Image acquisition}

Scan sessions consisted of an eyes-closed resting-state. Participants were instructed to simply rest with their eyes closed without sleeping or thinking about anything in particular. Images were acquired on a 1.5T scanner (Siemens Avanto; Erlangen, Germany). Participants were placed in a supine position in the MRI scanner, and their heads were immobilized with cushions to reduce motion artifacts. For the rs-fMRI, a total of 200 volumes were recorded using a gradientecho T2*-weighted echo-planar imaging sequence (TR, 2000 ms; TE, 48 ms; matrix, 64 x 64; voxel size, 3.5 x $3.5 \mathrm{~mm}$; flip angle, $90^{\circ}$; slice thickness, $4 \mathrm{~mm}$; slice gap, $0.8 \mathrm{~mm}$ ). We acquired 24 interleaved axial slices parallel to the anterior-posterior commissure plane covering the entire brain. The total rs-fMRI scan length was 6:40 minutes. Prior to the rs-fMRI sequences, structural images were acquired using a high-resolution T1-weighted MPRAGE sequence with TR/TE $=$ 
$2200 / 3.79 \mathrm{~ms}$, flip angle $15^{\circ}$, voxel size $=1 \times 1 \times 1 \mathrm{~mm}$, which facilitated the localization and co-registration of functional data.

\section{Image preprocessing}

We used the Data Processing Assistant for Resting-State (DPARSF, http://rfmri.org/DPARSF), which is a plug-in software within the Data Processing \& Analysis for Brain Imaging (DPABI V2.0_151201, http://rfmri.org/dpabi; Yan, Wang, Zuo, \& Zang, 2016), based on Statistical Parametric Mapping (SPM, http://www.fil.ion.ucl.ac.uk/spm), to carry out rsfMRI data processing. Preprocessing included the following steps: 1) the first five volumes of each raw rs-fMRI dataset were discarded to allow for T1 equilibration; 2) slice timing correction for interleaved acquisitions (the middle slice was used as the reference point); 3) realignment using a six-parameter (rigid body) linear transformation with a two-pass procedure (registered to the first image and then registered to the mean of the images after the first realignment); 4) coregistration of the individual structural images (T1-weighted MPRAGE) to the mean functional image; 5) segmentation of structural images into gray matter (GM), white matter (WM), and cerebrospinal fluid (CSF) using the Diffeomorphic Anatomical Registration Through Exponentiated Lie algebra (DARTEL) tool (Ashburner, 2007); 6) removal of spurious variance through linear regression: 24 parameters from the realignment step (6 head motion parameters, 6 head motion parameters one time point before, and the 12 corresponding squared items; Friston, Williams, Howard, Frackowiak, \& Turner, 1996), scrubbing within regression (spike regression as well as 1 back and 2 forward neighbors; Yan, Craddock, He, \& Milham, 2013) at framewise displacement of (FD)>0.2mm (Power, Barnes, Snyder, Schlaggar, \& Petersen, 2012), linear and quadratic trends, the WM signal (segmentation mask thresholded at 90\% probability), and the CSF signal (segmentation mask thresholded at $70 \%$ probability); 7) spatial normalization to the

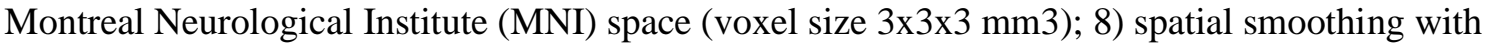
a 4 mm FWHM Gaussian Kernel; and 9) band-pass temporal filtering (0.01-0.1 Hz) to reduce the effect of low frequency drift and high frequency noise (Biswal, Zerrin Yetkin, Haughton, \& Hyde, 1995; Lowe, Mock, \& Sorenson, 1998).

Participants with more than $1 \mathrm{~mm} /$ degree of movement in any of the six directions or a large removal of data points during scrubbing regression (more than 50 volumes, ensuring at least 5 minutes of remaining data) were excluded from the analyses. In the final sample, descriptive statistics for the head motion metrics provided by DPARSFA were as follows: mean RMS (mean $=0.11, \mathrm{SD}=0.04$, range $=0.04-0.26$ ), mean FD Power $(\mathrm{mean}=0.12, \mathrm{SD}=0.03$, range $=0.05$ 0.20 ), mean FD Jenkinson (mean $=0.06, \mathrm{SD}=0.02$, range $=0.03-0.10$ ), and mean FD Van Dijk (mean $=0.03, \mathrm{SD}=0.01$, range $=0.01-0.07)$. 


\section{Resting-State Functional Connectivity analysis}

A seed-based correlation approach between a priori regions of interest (ROIs) was performed to investigate the link between reward sensitivity and the rs-FC between the main areas of the brain reward system. In this method, FC relies on the correlation between the averaged BOLD signal of a ROI, also called the seed, and the BOLD signals of other parts of the brain (voxels or other ROIs). For this study, we defined seeds for four BAS-related areas involved in motivational and emotional processes (Haber \& Knutson, 2010; McNaughton \& Corr, 2008): the ventral tegmental area (VTA), the ventral striatum (VS), the anterior cingulate cortex (ACC), and the ventromedial prefrontal cortex (vmPFC). The seeds for the ACC and vmPFC (gyrus rectus plus medial orbital frontal cortex) were defined with the anatomical automatic labeling (AAL) template (Tzourio-Mazoyer et al., 2002). Unfortunately, this atlas does not include ROIs for VTA and VS. Thus, we used other atlases for these ROIs. For the VTA, we used a published probabilistic atlas provided by Murty et al. (2014) at a probability threshold of $80 \%$, whereas for the VS we used a published atlas provided by Tziortzi et al. (2014), taking the ventral striatal region defined within the "limbic network". All the seeds were included bilaterally (see Figure $1)$.

After seed definition, the rs-FCs between the ROIs were investigated through pairwise correlations between the time-series of the seeds. After the correlations, Fisher's r-to- $Z$ transformation was performed to normalize the variance in correlation values.

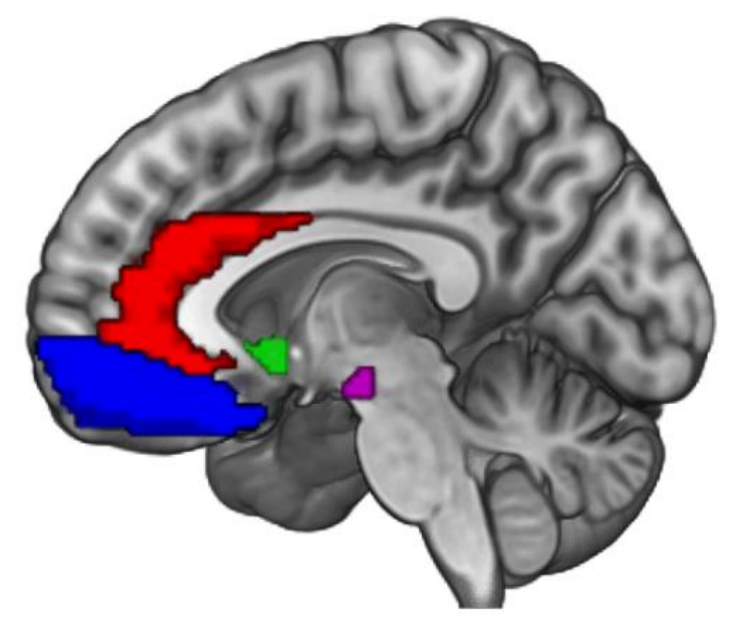

Figure 1. Seed ROIs included in the rs-FC analyses. Red: anterior cingulate cortex; Blue: ventromedial prefrontal cortex; Green: ventral striatum; Purple: ventral tegmental area. 
Statistical analyses were performed using SPSS Statistics for Windows, Version 25.0 (Armonk, NY: IBM Corp.). First, we carried out a one-sample $t$ test analysis on the framework of the general linear model using the $\mathrm{z}$ scores for each pair of ROIs as the dependent variable. Afterwards, in order to investigate how the connectivity between the a priori seeds was related to reward sensitivity, partial correlation analyses between the SR scores and the $\mathrm{z}$ scores were performed. Effects of age and gender were regressed out in all the analyses. The statistical significance threshold for all the analyses was set at $p<0.05$ FDR-corrected (Benjamini \& Hochberg, 1995) using a syntax script for SPSS (http://www01.ibm.com/support/docview.wss?uid=swg21476447). Because we took four ROIs as seeds, six comparisons were carried out.

\section{Results}

\section{Seed-based connectivity analyses}

One-sample t test analyses revealed a significant FC between all the seed time series used in the study (Table 1), thus resulting in an interconnected network at rest ( $\mathrm{p}<.05$ FDR-corrected). All the FCs resulted in positive values, except for the vmPFC-VTA FC.

To investigate personality effects of the rs-FCs, partial correlations were carried out by taking rs-FCs between ROIs and scores on the SR scale. A significant effect was found in the rsFC between the VTA-vmPFC ( $p<.05$ FDR-corrected), showing an increase in the connectivity strength between the two structures. Furthermore, we also observed a stronger connectivity between the vmPFC-ACC $(\mathrm{p}<.05$ FDR-corrected). Partial correlation analysis details are reported in Table 2 and Figure 2.

Moreover, because the ROIs selected as seeds differed considerably in size (mainly between cortical and subcortical regions), we performed further analyses to check that our results were not affected by different signal-to-noise levels (see details in Supplementary Material). 
Table 1. Results from the one-sample $t$ test (controlling for age and gender) on the Fisher's z correlation coefficients (as a measure of connectivity).

\begin{tabular}{|c|c|c|c|c|c|c|c|c|}
\hline & & & & & & & $95 \%$ & \\
\hline & $\mathrm{t}$ & Sig. (2-tailed) & Mean & Std. Deviation & Min. & Max. & Confide & Intervals \\
\hline & & & & & & & Inferior & Superior \\
\hline ACC-vmPFC & 17.06 & $.000 *$ & .38 & .21 & -.24 & .92 & .34 & .42 \\
\hline ACC-VS & 14.75 & $.000 *$ & .31 & .20 & -.16 & .72 & .27 & .35 \\
\hline ACC-VTA & 7.29 & $.000 *$ & .11 & .15 & -.27 & .44 & .08 & .14 \\
\hline VS-vmPFC & 9.98 & $.000 *$ & .23 & .22 & -.18 & .88 & .19 & .28 \\
\hline VS-VTA & 5.07 & $.000 *$ & .10 & .18 & -.40 & .63 & .06 & .13 \\
\hline vmPFC-VTA & -2.65 & $.010 *$ & -.06 & .20 & -.60 & .54 & -.10 & -.01 \\
\hline
\end{tabular}

* FDR-corrected at $\mathrm{p}<.05 ; \mathrm{ACC}=$ anterior cingulate cortex, $\mathrm{vmPFC}=$ ventromedial prefrontal cortex, $\mathrm{VS}=\mathrm{ventral}$ striatum (bilateral), VTA=ventral tegmental area.

Table 2. Partial correlations (controlling for age and gender) between SR scores and Fisher's z correlation coefficients among seed time series.

\begin{tabular}{llllllll}
\hline & & ACC-vmPFC & ACC-VS & ACC-VTA & VS-vmPFC & VS-VTA & vmPFC-VTA \\
\hline SR & Corr. & .257 & -.029 & .086 & -.117 & .117 & .257 \\
& Sig. & $.016^{*}$ & .792 & .427 & .279 & .281 & $.016^{*}$ \\
\hline
\end{tabular}

* FDR-corrected at $\mathrm{p}<.05$; ACC=anterior cingulate cortex, vmPFC=ventromedial prefrontal cortex, $\mathrm{VS}=$ ventral striatum (bilateral), VTA=ventral tegmental area.
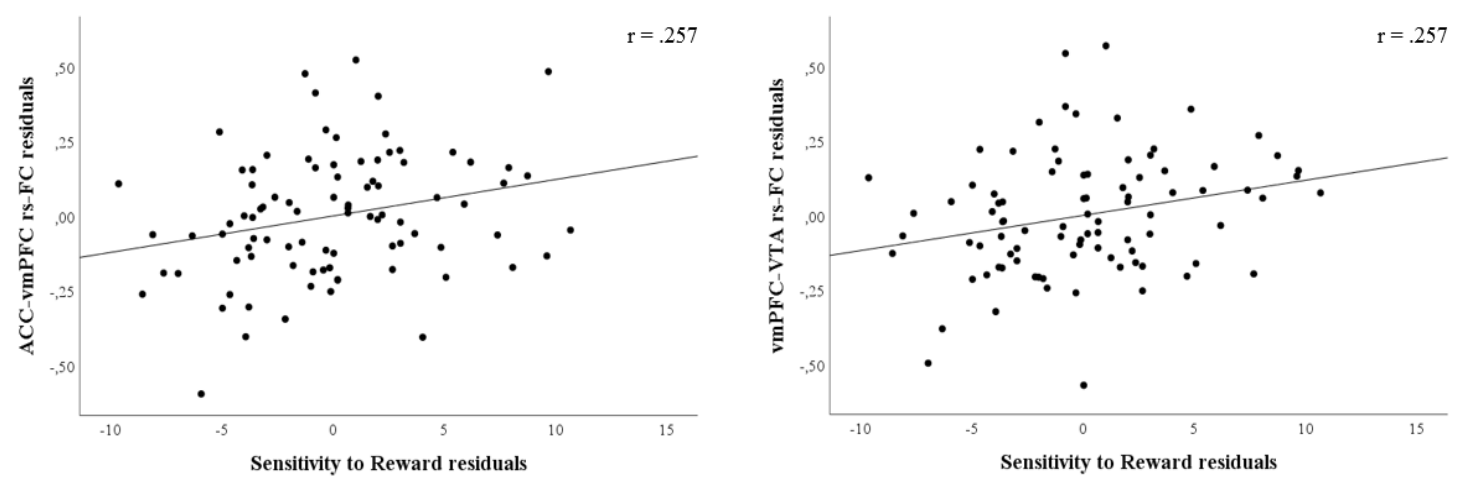

Figure 2. Scatterplots of residuals illustrating the partial correlations of ACC-vmPFC and vmPFC-VTA rs-FCs with SR scores after regressing out age and gender in each variable. 


\section{Discussion}

In this study we analyzed the relationship between reward sensitivity, as a measure of BAS activity, and the rs-FC between key structures within the brain reward system in healthy adults. With one exception (the rs-FC between the vmPFC and VTA), our results showed a significant positive and moderate interconnectivity between all the areas included in the study, thus providing information about the neural coupling of BAS-related areas at rest. Furthermore, as expected, we observed a connection between the reward sensitivity trait and the rs-FC between areas of the BAS. Specifically, we observed a positive correlation between the scores on the SR scale and the rs-FC between the ACC and vmPFC and the vmPFC and VTA. Thus, the individual differences in BAS activity were associated with a different, stable connectivity between the brain areas involved in reward detection and processing.

In our study, we delimited four regions of interest related to the BAS associated with emotional and motivational processing: the VTA, the VS, the ACC, and the vmPFC. As expected, the results of this study corroborated that the activity of these areas during resting-state is moderately coupled. This is especially true between the last three ROIs, showing that the activity of the VS, vmPFC, and ACC is closely interrelated. Previous resting-state fMRI studies have shown a strong connectivity between the VS and the vmPFC and ACC (Cauda et al., 2011; Choi, Yeo, \& Buckner, 2012; Di Martino et al., 2008), and animal and human studies have suggested that this relationship mediates the processing of rewards (Pujara, Philippi, Motzkin, Baskaya, \& Koenigs, 2016; Richard \& Berridge, 2013; Smith \& Graybiel, 2013). In addition, the vmPFC and the ACC are functionally interrelated, as found in previous studies (Bzdok et al., 2013; Margulies et al., 2007), supporting the evaluation function of emotional and social stimuli (Bzdok et al., 2013). Although we obtained a strong interconnectivity between these areas, our results showed that the values of rs-FCs decreased when the VTA was involved (especially with ACC and vmPFC). The VTA is a small region that is of great relevance in processing reward stimuli (D’Ardenne, McClure, Nystrom, \& Cohen, 2008; Düzel et al., 2009; Hayes, Duncan, Xu, \& Northoff, 2014), but the cortical areas are bigger and receive influence from a large number of brain structures. Hence, this could be one of the reasons we observed a negative correlation between the VTA and vmPFC. Nonetheless, even in this negative correlation, the range of rs-FCs is wide. Therefore, some individuals presented a strong positive coupling between the VTA and cortical areas, whereas others presented negatively correlated activity between these two structures.

We also hypothesized that SR scores would be associated with the rs-FC between the BAS-related structures. According to this hypothesis, SR scores would be positively correlated with the rs-FC between the vmPFC (the medial segment of the OFC cortex) and the VTA, and 
between the vmPFC and the ACC. Both the vmPFC and VTA are connected via the mesocortical pathway, a dopaminergic branch responsible for initiating motivated reward-based approach behaviors (Arias-Carrión, Stamelou, Murillo-Rodríguez, Menéndez-González, \& Pöppel, 2010; D'Ardenne et al., 2008; Haber \& Knutson, 2010). The VTA has largely been described as the main component of the reward brain circuitry because the mesocortical and mesolimbic pathways arise from this structure (Arias-Carrión et al., 2010; Krebs, Schott, \& Düzel, 2009). Furthermore, the dopamine cells in the VTA are involved in motivational salience and value (Bromberg-Martin, Matsumoto, \& Hikosaka, 2010; Depue \& Collins, 1999; Hayes et al., 2014), thus promoting reward approach and consumption, which is the "wanting" component of reward-based behaviors (Berridge, Robinson, \& Aldridge, 2009). In addition to the VTA, the vmPFC is also a key region of the brain reward network and the principal projection from the VTA within the mesocortical pathway. Similarly to the VTA, the vmPFC has also been involved in reward processing, mainly in reward assessment and decision-making (Hayes et al., 2014; Rangel, Camerer, \& Montague, 2008; Sescousse et al., 2013). Importantly, one of the factors that makes the vmPFC involved in reward-related decision-making stems from the modulatory effect on dopaminergic neurotransmission. Previous studies identified an inhibitory pathway between the medial prefrontal cortex and the VTA through glutamatergic activation of GABA interneurons in the VTA, thus reducing the levels of dopamine in the VS (mesolimbic pathway) (Carr \& Sesack, 2000; Sesack, Carr, Omelchenko, \& Pinto, 2003).

The results obtained are consistent with previous proposals relating reward sensitivity (Pickering \& Gray, 2001) and extraversion (Depue \& Collins, 1999; Pickering \& Gray, 1999) to dopaminergic transmission from the VTA. Individual differences in the dopaminergic transmission from the VTA via the mesocortical pathway may be responsible for a different ability to detect and evaluate rewarding stimuli. Interestingly, our results coincide with these previous reports, as we observed greater connectivity between the primary mesocortical areas during a resting-state condition (absence of stimuli), that is, during one's default basal activity. Therefore, the stronger rs-FC between the VTA and vmPFC may predispose the person to interpret more situations as rewarding. Importantly, the FC connectivity between the two areas is reversed when an incentive is detected (Costumero et al., 2013a). In the study by Costumero et al. (2013a), the authors compared the neural processing of low and high magnitude incentives as a function of reward sensitivity. Rewarding stimuli activated the VTA and VS significantly more in individuals with higher scores on the SR scale, but the connectivity between the VTA and vmPFC during high incentive stimuli was reduced in high SR individuals. Overall, these results may suggest that the reward sensitivity trait would be directly related to the connectivity between the VTA and vmPFC and, therefore, to the proneness to detecting (positive correlation under absence of stimuli) and responding (negative correlation under presence of stimuli) to rewarding 
stimuli. However, this hypothesis is speculative, and further studies are need to investigate the relationship between BAS activity and VTA-vmPFC connectivity.

Our results also showed a positive correlation between SR scores and rs-FC between the ACC and vmPFC. Both are dopaminergic-innervated adjacent structures within the reward brain circuitry, and they are involved in evaluating, managing, and selecting rewarding situations. Global results showed a stronger rs-FC between them in the absence of a task, but previous literature has demonstrated this strong FC during reinforcement-guided decision making (Fatahi, Haghparast, Khani, \& Kermani, 2018). The role of the two structures is different: whereas the ACC is relevant for reward prediction and prediction error encoding, the vmPFC is more involved in outcome evaluation, establishing stimulus-outcome associations and encoding rewarding features of a stimulus (Rushworth, Noonan, Boorman, Walton, \& Behrens, 2011). The main dissociation is that the vmPFC encodes outcomes predicted by the ACC, but it also encodes unexpected rewarding outcomes not predicted by the ACC (Vassena, Krebs, Silvetti, Fias, \& Verguts, 2014). In relation to our results, we observed stronger connectivity at rest between these structures associated with SR, thus suggesting a tendency to predict contingencies associated with emotional stimuli. Thus, the increased rs-FC observed in participants with high SR scores may serve to establish more frequent predictions of positive outcomes when processing reward cues and evaluate these outcomes as more positive.

One of the relevant results of this study is that the influence of reward sensitivity on rsFC within the reward network is more focused on the mesocortical pathway than on the mesolimbic pathway. Contrary to previous observations investigating reward processing as a function of reward sensitivity, where the mesolimbic and mesocortical pathways are relevant (Costumero et al., 2013a; Costumero et al., 2013b, for a review see Kennis, Rademaker, \& Geuze, 2013), the absence of emotional stimuli seems to move the focus of attention to the mesocortical pathway. The results of a recent study are consistent with this pattern (Richter \& Gruber, 2018). This study compared the brain areas involved in processing salient infrequent stimuli to those involved in processing salient rewarding stimuli. Results showed that both kinds of stimuli activated the VTA and the VS, but the PPI analysis revealed a different FC of the VTA: whereas reward stimuli only co-activated the VS, salient non-rewarding stimuli showed a FC of the VTA with both vmPFC and ACC. Similarly, our results revealed higher coupling of the VTA and vmPFC during a resting-state condition in participants with high reward sensitivity. Therefore, the reward sensitivity trait would be related to brain areas specialized in processing salient stimuli (and not only salient rewarding stimuli). Overall, this finding suggests that the mesocortical pathway is more involved in detecting salient stimuli, and the role of the vmPFC/ACC areas should be more related to regulating responses to these stimuli. 
The present results are aligned with previous literature showing that reward sensitivity is associated with differences in cognitive processing. On different tasks, previous studies showed that reward sensitivity was positively associated with more cognitive flexibility on tasks requiring fast, automatic processing (Avila et al., 2003; Avila \& Parcet, 1997) and better cognitive focusing on tasks requiring slow, conscious processing (Avila, 1995; Ávila \& Parcet, 2002). These tasks reflected differences in the capacity to process stimuli that, in fact, would determine a different capacity to detect rewarding stimuli. The results of the present study seem to suggest that these differences would be more related to the mesocortical pathway.

In sum, the present study adds to the extensive literature testing for neural differences associated with individual differences in BAS activity. All this literature has shown that reward sensitivity is associated with structural differences in frontostriatal areas and with enhanced responses to reward stimuli in the mesolimbic network. However, the RST model also predicts that a personality trait, such as reward sensitivity, would also determine a different functioning in detecting possible rewarding stimuli (Avila, Parcet, \& Barrós-Loscertales, 2008; Derryberry \& Reed, 1994). The present study has focused these differences on the mesocortical dopaminergic pathway, which could act as a global vigilance system prepared to detect reward stimuli.

Finally, our study presents some limitations. One of them is related to the rs-FC approach to the research question. Based on our a priori ROIs, we decided to use a seed-based (pairwise) correlation approach, as opposed to other useful methods for studying rs-FC, such as seed-based whole-brain FC or independent component analysis (ICA). Thus, although our method was more appropriate for testing specific hypotheses, these methods could provide further results not observed with our approach. Regarding our ROIs, another limitation would be related to their location, as the VTA and vmPFC are located at the ventral edge of the brain, making them vulnerable to the nuisance of susceptibility artefacts. However, this problem is inherent to all the fMRI, and new methods should be implemented to improve this aspect. Moreover, the sample selected for this study could also be a limitation. Although we recruited participants from a community sample, the majority of our participants were undergraduates, thus resulting in a relatively young sample (i.e. mean of 22.4 years). Even though this is not a problem in itself, it could impede the generalization of the results to older populations. In addition, although the aim of this study was not to analyze sex differences in the rs-FC linked to reward sensitivity, future studies should investigate this subject because recent data have revealed differences in the rs-FC between males and females (Nostro et al., 2018). 


\section{Acknowledgements}

This work was supported by grants from Generalitat Valenciana (PROMETEO/2017/109) and Ministerio de Economía y Competitividad (PSI2016-78805-R) to C.A. In addition, this work was supported by a pre-doctoral graduate program grant (National FPU to J.A.-V.) and a post-doctoral graduate program grant (Juan de la Cierva to V.C.).

\section{References}

Aarts, E., van Holstein, M., \& Cools, R. (2011). Striatal dopamine and the interface between motivation and cognition. Frontiers in Psychology, 2, 163. doi:https://doi.org/10.3389/fpsyg.2011.00163

Aluja, A. (2004). Sensitivity to punishment, sensitivity to reward and sexuality in females. Personality and Individual Differences, 36(1), 5-10. doi:https://doi.org/10.1016/S01918869(02)00219-2

Angelides, N. H., Gupta, J., \& Vickery, T. J. (2017). Associating resting-state connectivity with trait impulsivity. Social Cognitive and Affective Neuroscience, 12(6), 1001-1008. doi:https://doi.org/10.1093/scan/nsx031

Arias-Carrión, O., Stamelou, M., Murillo-Rodríguez, E., Menéndez-González, M., \& Pöppel, E. (2010). Dopaminergic reward system: a short integrative review. International Archives of Medicine, 3, 24. doi:https://doi.org/10.1186/1755-7682-3-24

Ashburner, J. (2007). A fast diffeomorphic image registration algorithm. NeuroImage, 38(1), 95113. doi:https://doi.org/10.1016/J.NEUROIMAGE.2007.07.007

Avila, C. (1995). Facilitation and inhibition of visual orienting as a function of personality. Personality and Individual Differences, 18(4), 503-509. doi:https://doi.org/10.1016/01918869(94)00184-T

Avila, C. (2001). Distinguishing BIS-mediated and BAS-mediated disinhibition mechanisms: a comparison of disinhibition models of Gray $(1981,1987)$ and of Patterson and Newman (1993). Journal of Personality and Social Psychology, 80(2), 311-324. doi:https://doi.org/10.1037/00223514.80.2.311

Avila, C., Barrós, A., Ortet, G., Antònia Parcet, M., \& Ibañez, M. I. (2003). Set-shifting and sensitivity to reward: A possible dopamine mechanism for explaining disinhibitory disorders. Cognition \& Emotion, 17(6), 951-959. doi:https://doi.org/10.1080/02699930302314 
Avila, C., Garbin, G., Sanjuán, A., Forn, C., Barrós-Loscertales, A., Bustamante, J. C., ... Parcet, M. A. (2012). Frontostriatal response to set switching is moderated by reward sensitivity. Social Cognitive and Affective Neuroscience, 7(4), 423-430. doi:https://doi.org/10.1093/scan/nsr028

Avila, C., Moltó, J., \& Segarra, P. (1995). Sensitivity to conditioned or unconditioned stimuli: What is the mechanism underlying passive avoidance deficits in extraverts? Journal of Research in Personality, 29(4), 373-394. doi:https://doi.org/10.1006/JRPE.1995.1022

Avila, C., \& Parcet, M. A. (1997). Impulsivity and anxiety differences in cognitive inhibition. Personality and Individual Differences, 23(6), 1055-1064. doi:https://doi.org/10.1016/S01918869(97)00124-4

Avila, C., \& Parcet, M. A. (2000). The role of Gray's impulsivity in anxiety-mediated differences in resistance to extinction. European Journal of Personality, 14(3), 185-198. doi:https://doi.org/10.1002/1099-0984(200005/06)14:3<185::AID-PER370>3.0.CO;2-U

Ávila, C., \& Parcet, M. A. (2002). Individual differences in reward sensitivity and attentional focus. Personality and Individual Differences, 33(6), 979-996. doi:https://doi.org/10.1016/S0191-8869(01)00207-0

Avila, C., Parcet, M. A., \& Barrós-Loscertales, A. (2008). A cognitive neuroscience approach to individual differences in sensitivity to reward. Neurotoxicity Research, 14(2-3), 191-203. doi:https://doi.org/10.1007/BF03033810

Avila, C., Parcet, M. A., Ortet, G., \& Ibáñez-Ribes, M. I. (1999). Anxiety and counterconditioning: the role of the behavioral inhibition system in the ability to associate aversive stimuli with future rewards. Personality and Individual Differences, 27(6), 1167-1179. doi:https://doi.org/10.1016/S0191-8869(99)00060-4

Ávila, C., \& Torrubia, R. (2004). Personality, expectations, and response strategies in multiplechoice question examinations in university students: a test of Gray's hypotheses. European Journal of Personality, 18(1), 45-59. doi:https://doi.org/10.1002/per.506

Ávila, C., \& Torrubia, R. (2008). Performance and conditioning studies. In P. J. Corr (Ed.), The reinforcement sensitivity theory of personality (pp. 228-260). New York: Cambridge University Press.

Barrós-Loscertales, A., Meseguer, V., Sanjuán, A., Belloch, V., Parcet, M. A., Torrubia, R., \& Avila, C. (2006). Striatum gray matter reduction in males with an overactive behavioral activation system. The European Journal of Neuroscience, 24(7), 2071-2074. doi:https://doi.org/10.1111/j.1460-9568.2006.05084.x 
Barrós-Loscertales, A., Ventura-Campos, N., Sanjuán-Tomás, A., Belloch, V., Parcet, M.-A., \& Avila, C. (2010). Behavioral activation system modulation on brain activation during appetitive and aversive stimulus processing. Social Cognitive and Affective Neuroscience, 5(1), 18-28. doi:https://doi.org/10.1093/scan/nsq012

Beaver, J. D., Lawrence, A. D., van Ditzhuijzen, J., Davis, M. H., Woods, A., \& Calder, A. J. (2006). Individual differences in reward drive predict neural responses to images of food. The Journal of Neuroscience, 26(19), 5160-5166. doi:https://doi.org/10.1523/JNEUROSCI.035006.2006

Benjamini, Y., \& Hochberg, Y. (1995). Controlling the false discovery rate: A practical and powerful approach to multiple testing. Journal of the Royal Statistical Society. Series B (Methodological), 57(1), 289-300. doi:https://doi.org/10.2307/2346101

Berridge, K. C., Robinson, T. E., \& Aldridge, J. W. (2009). Dissecting components of reward: "liking", "wanting", and learning. Current Opinion in Pharmacology, 9(1), 65-73. doi:https://doi.org/10.1016/j.coph.2008.12.014

Bijttebier, P., Beck, I., Claes, L., \& Vandereycken, W. (2009). Gray’s Reinforcement Sensitivity Theory as a framework for research on personality-psychopathology associations. Clinical Psychology Review, 29(5), 421-430. doi:https://doi.org/10.1016/j.cpr.2009.04.002

Biswal, B., Zerrin Yetkin, F., Haughton, V. M., \& Hyde, J. S. (1995). Functional connectivity in the motor cortex of resting human brain using echo-planar mri. Magnetic Resonance in Medicine, 34(4), 537-541. doi:https://doi.org/10.1002/mrm.1910340409

Boog, M., Goudriaan, A. E., Wetering, B. J. M. V. D., Polak, M., Deuss, H., \& Franken, I. H. A. (2014). Rash impulsiveness and reward sensitivity as predictors of treatment outcome in male substance dependent patients. Addictive Behaviors, 39(11), 1670-1675. doi:https://doi.org/10.1016/J.ADDBEH.2014.02.020

Bromberg-Martin, E. S., Matsumoto, M., \& Hikosaka, O. (2010). Dopamine in motivational control: Rewarding, aversive, and alerting. Neuron, 68(5), 815-834. doi:https://doi.org/10.1016/J.NEURON.2010.11.022

Bzdok, D., Langner, R., Schilbach, L., Engemann, D. A., Laird, A. R., Fox, P. T., \& Eickhoff, S. B. (2013). Segregation of the human medial prefrontal cortex in social cognition. Frontiers in Human Neuroscience, 7, 232. doi:https://doi.org/10.3389/fnhum.2013.00232

Carr, D. B., \& Sesack, S. R. (2000). Projections from the rat prefrontal cortex to the ventral tegmental area: target specificity in the synaptic associations with mesoaccumbens and 
mesocortical neurons. The Journal of Neuroscience, 20(10), 3864-3873. doi:https://doi.org/10.1523/JNEUROSCI.20-10-03864.2000

Carter, R. M., Macinnes, J. J., Huettel, S. A., \& Adcock, R. A. (2009). Activation in the VTA and nucleus accumbens increases in anticipation of both gains and losses. Frontiers in Behavioral Neuroscience, 3, 21. doi:https://doi.org/10.3389/neuro.08.021.2009

Carver, C. S., \& White, T. L. (1994). Behavioral inhibition, behavioral activation, and affective responses to impending reward and punishment: The BIS/BAS Scales. Journal of Personality and Social Psychology, 67(2), 319-333. doi:https://doi.org/10.1037/0022-3514.67.2.319

Caseras, X., Àvila, C., \& Torrubia, R. (2003). The measurement of individual differences in Behavioural Inhibition and Behavioural Activation Systems: a comparison of personality scales. Personality and Individual Differences, 34(6), 999-1013. doi:https://doi.org/10.1016/S01918869(02)00084-3

Cauda, F., Cavanna, A. E., D’agata, F., Sacco, K., Duca, S., \& Geminiani, G. C. (2011). Functional connectivity and coactivation of the nucleus accumbens: A combined functional connectivity and structure-based meta-analysis. Journal of Cognitive Neuroscience, 23(10), 2864-2877. doi:https://doi.org/10.1162/jocn.2011.21624

Choi, E. Y., Yeo, B. T. T., \& Buckner, R. L. (2012). The organization of the human striatum estimated by intrinsic functional connectivity. Journal of Neurophysiology, 108(8), 2242-2263. doi:https://doi.org/10.1152/jn.00270.2012

Corr, P. J. (2004). Reinforcement sensitivity theory and personality. Neuroscience and Biobehavioral Reviews, 28(3), 317-332. doi:https://doi.org/10.1016/j.neubiorev.2004.01.005

Costumero, V., Barrós-Loscertales, A., Bustamante, J. C., Ventura-Campos, N., Fuentes, P., \& Ávila, C. (2013a). Reward sensitivity modulates connectivity among reward brain areas during processing of anticipatory reward cues. The European Journal of Neuroscience, 38(3), 23992407. doi:https://doi.org/10.1111/ejn.12234

Costumero, V., Barrós-Loscertales, A., Bustamante, J. C., Ventura-Campos, N., Fuentes, P., Rosell-Negre, P., \& Ávila, C. (2013b). Reward sensitivity is associated with brain activity during erotic stimulus processing. PLoS One, 8(6), e66940. doi:https://doi.org/10.1371/journal.pone.0066940

Costumero, V., Barrós-Loscertales, A., Fuentes, P., Rosell-Negre, P., Bustamante, J. C., \& Ávila, C. (2016). BAS-drive trait modulates dorsomedial striatum activity during reward responseoutcome associations. Brain Imaging and Behavior, 10(3), 869-879. doi:https://doi.org/10.1007/s11682-015-9466-5 
D’Ardenne, K., McClure, S. M., Nystrom, L. E., \& Cohen, J. D. (2008). BOLD responses reflecting dopaminergic signals in the human ventral tegmental area. Science, 319(5867), 12641267. doi:https://doi.org/10.1126/science.1150605

Depue, R. A., \& Collins, P. F. (1999). Neurobiology of the structure of personality: Dopamine, facilitation of incentive motivation, and extraversion. Behavioral and Brain Sciences, 22(3), 491569. doi:https://doi.org/10.1017/S0140525X99002046

Derryberry, D., \& Reed, M. A. (1994). Temperament and attention: Orienting toward and away from positive and negative signals. Journal of Personality and Social Psychology, 66(6), 11281139. doi:https://doi.org/10.1037/0022-3514.66.6.1128

Di Martino, A., Scheres, A., Margulies, D. S., Kelly, A. M. C., Uddin, L. Q., Shehzad, Z., ... Milham, M. P. (2008). Functional connectivity of human striatum: A resting state fMRI study. Cerebral Cortex, 18(12), 2735-2747. doi:https://doi.org/10.1093/cercor/bhn041

Düzel, E., Bunzeck, N., Guitart-Masip, M., Wittmann, B., Schott, B. H., \& Tobler, P. N. (2009). Functional imaging of the human dopaminergic midbrain. Trends in Neurosciences, 32(6), 321328. doi:https://doi.org/10.1016/J.TINS.2009.02.005

Fatahi, Z., Haghparast, A., Khani, A., \& Kermani, M. (2018). Functional connectivity between anterior cingulate cortex and orbitofrontal cortex during value-based decision making. $\begin{array}{llllll}\text { Neurobiology of } & \text { Learning }\end{array}$ doi:https://doi.org/10.1016/J.NLM.2017.11.014

Franken, I. H. A., \& Muris, P. (2006). BIS/BAS personality characteristics and college students' substance use. Personality and Individual Differences, 40(7), 1497-1503. doi:https://doi.org/10.1016/j.paid.2005.12.005

Franken, I. H. A., Muris, P., \& Georgieva, I. (2006). Gray's model of personality and addiction. Addictive Behaviors, 31(3), 399-403. doi:https://doi.org/10.1016/j.addbeh.2005.05.022

Friston, K. J., Williams, S., Howard, R., Frackowiak, R. S. J., \& Turner, R. (1996). Movementrelated effects in fMRI time-series. Magnetic Resonance in Medicine, 35(3), 346-355. doi:https://doi.org/10.1002/mrm.1910350312

Fuentes-Claramonte, P., Ávila, C., Rodríguez-Pujadas, A., Costumero, V., Ventura-Campos, N., Bustamante, J. C., ... Barrós-Loscertales, A. (2016a). Characterizing individual differences in reward sensitivity from the brain networks involved in response inhibition. NeuroImage, 124(Pt A), 287-299. doi:https://doi.org/10.1016/j.neuroimage.2015.08.067

Fuentes-Claramonte, P., Ávila, C., Rodríguez-Pujadas, A., Costumero, V., Ventura-Campos, N., Bustamante, J. C., ... Barrós-Loscertales, A. (2016b). Inferior frontal cortex activity is modulated 
by reward sensitivity and performance variability. Biological Psychology, 114, 127-137. doi:https://doi.org/10.1016/j.biopsycho.2016.01.001

Fuentes-Claramonte, P., Ávila, C., Rodríguez-Pujadas, A., Ventura-Campos, N., Bustamante, J. C., Costumero, V., ... Barrós-Loscertales, A. (2015). Reward sensitivity modulates brain activity in the prefrontal cortex, ACC and striatum during task switching. PLoS One, 10(4), e0123073. doi:https://doi.org/10.1371/journal.pone.0123073

Fuentes, P., Barrós-Loscertales, A., Rodríguez-Pujadas, A., Ventura-Campos, N., Bustamante, J. C., Costumero, V., ... Ávila, C. (2014a). Behavioral activation system modulation of brain activity during task switching. Personality and Individual Differences, 60, S52. doi:https://doi.org/10.1016/J.PAID.2013.07.212

Fuentes, P., Barrós-Loscertales, A., Rodríguez-Pujadas, A., Ventura-Campos, N., Bustamante, J. C., Costumero, V., ... Ávila, C. (2014b). Reward sensitivity modulation of brain activity during response inhibition. Personality and Individual Differences, $60, \quad$ S69. doi:https://doi.org/10.1016/J.PAID.2013.07.303

Glashouwer, K. A., Bloot, L., Veenstra, E. M., Franken, I. H. A., \& de Jong, P. J. (2014). Heightened sensitivity to punishment and reward in anorexia nervosa. Appetite, 75, 97-102. doi:https://doi.org/10.1016/j.appet.2013.12.019

Gray, J. A. (1982). The neuropsychology of anxiety: An enquiry into the functions of the septohippocampal system. Oxford: Oxford University Press.

Gray, J. A., \& McNaughton, N. J. (2000). The neuropsychology of anxiety: An inquiry into the functions of the septo-hippocampal system (2nd ed). Oxford: Oxford University Press.

Haber, S. N., \& Knutson, B. (2010). The reward circuit: linking primate anatomy and human imaging. Neuropsychopharmacology, 35(1), 4-26. doi:https://doi.org/10.1038/npp.2009.129

Hahn, T., Dresler, T., Ehlis, A.-C., Plichta, M. M., Heinzel, S., Polak, T., ... Fallgatter, A. J. (2009). Neural response to reward anticipation is modulated by Gray's impulsivity. NeuroImage, 46(4), 1148-1153. doi:https://doi.org/10.1016/j.neuroimage.2009.03.038

Hayes, D. J., Duncan, N. W., Xu, J., \& Northoff, G. (2014). A comparison of neural responses to appetitive and aversive stimuli in humans and other mammals. Neuroscience and Biobehavioral Reviews, 45, 350-368. doi:https://doi.org/10.1016/j.neubiorev.2014.06.018

Holmes, A. J., Hollinshead, M. O., Roffman, J. L., Smoller, J. W., \& Buckner, R. L. (2016). Individual differences in cognitive control circuit anatomy link sensation seeking, impulsivity, and substance use. The Journal of Neuroscience, 36(14), 4038-4049. doi:https://doi.org/10.1523/JNEUROSCI.3206-15.2016 
Kasch, K. L., Rottenberg, J., Arnow, B. A., \& Gotlib, I. H. (2002). Behavioral activation and inhibition systems and the severity and course of depression. Journal of Abnormal Psychology, 111(4), 589-597. doi:https://doi.org/10.1037/0021-843X.111.4.589

Kennis, M., Rademaker, A. R., \& Geuze, E. (2013). Neural correlates of personality: an integrative review. Neuroscience and Biobehavioral Reviews, 37(1), 73-95. doi:https://doi.org/10.1016/j.neubiorev.2012.10.012

Knyazev, G. G. (2004). Behavioural activation as predictor of substance use: mediating and moderating role of attitudes and social relationships. Drug and Alcohol Dependence, 75(3), 309321. doi:https://doi.org/10.1016/j.drugalcdep.2004.03.007

Krebs, R. M., Schott, B. H., \& Düzel, E. (2009). Personality traits are differentially associated with patterns of reward and novelty processing in the human substantia nigra/ventral tegmental area. Biological Psychiatry, 65(2), 103-110. doi:https://doi.org/10.1016/j.biopsych.2008.08.019

Lerner, D. A., Hatak, I., \& Rauch, A. (2018). Deep roots? Behavioral Inhibition and Behavioral Activation System (BIS/BAS) sensitivity and entrepreneurship. Journal of Business Venturing Insights, 9, 107-115. doi:https://doi.org/10.1016/J.JBVI.2018.02.005

Locke, H. S., \& Braver, T. S. (2008). Motivational influences on cognitive control: behavior, brain activation, and individual differences. Cognitive, Affective \& Behavioral Neuroscience, 8(1), 99-112. doi:https://doi.org/10.3758/CABN.8.1.99

Lowe, M. J., Mock, B. J., \& Sorenson, J. A. (1998). Functional Connectivity in Single and Multislice Echoplanar Imaging Using Resting-State Fluctuations. NeuroImage, 7(2), 119-132. doi:https://doi.org/10.1006/NIMG.1997.0315

Margulies, D. S., Kelly, A. M. C., Uddin, L. Q., Biswal, B. B., Castellanos, F. X., \& Milham, M. P. (2007). Mapping the functional connectivity of anterior cingulate cortex. NeuroImage, 37(2), 579-588. doi:https://doi.org/10.1016/j.neuroimage.2007.05.019

Matton, A., Goossens, L., Braet, C., \& Vervaet, M. (2013). Punishment and reward sensitivity: are naturally occurring clusters in these traits related to eating and weight problems in adolescents? European Eating Disorders Review, 21(3), 184-194. doi:https://doi.org/10.1002/erv.2226

Matton, A., Goossens, L., Vervaet, M., \& Braet, C. (2015). Temperamental differences between adolescents and young adults with or without an eating disorder. Comprehensive Psychiatry, 56, 229-238. doi:https://doi.org/10.1016/J.COMPPSYCH.2014.09.005 
McNaughton, N., \& Corr, P. (2008). The neuropsychology of fear and anxiety: A foundation for reinforcement sensitivity theory. In P. J. Corr (Ed.), The reinforcement sensitivity theory of personality (pp. 44-94). Cambridge: Cambridge University Press.

Mitchell, J. T., \& Nelson-Gray, R. O. (2006). Attention-deficit/hyperactivity disorder symptoms in adults: Relationship to Gray's behavioral approach system. Personality and Individual Differences, 40(4), 749-760. doi:https://doi.org/10.1016/j.paid.2005.08.011

Mortensen, J. A., Lehn, H., Evensmoen, H. R., \& Håberg, A. K. (2015). Evidence for an antagonistic interaction between reward and punishment sensitivity on striatal activity: A verification of the Joint Subsystems Hypothesis. Personality and Individual Differences, 74, 214 219. doi:https://doi.org/10.1016/J.PAID.2014.10.023

Murty, V. P., Shermohammed, M., Smith, D. V., Carter, R. M., Huettel, S. A., \& Adcock, R. A. (2014). Resting state networks distinguish human ventral tegmental area from substantia nigra. NeuroImage, 100, 580-589. doi:https://doi.org/10.1016/J.NEUROIMAGE.2014.06.047

Newman, J. P., MacCoon, D. G., Vaughn, L. J., \& Sadeh, N. (2005). Validating a distinction between primary and secondary psychopathy with measures of Gray's BIS and BAS constructs. Journal of Abnormal Psychology, 114(2), 319-323. doi:https://doi.org/10.1037/0021843X.114.2.319

Newman, J. P., Widom, C. S., \& Nathan, S. (1985). Passive avoidance in syndromes of disinhibition: psychopathy and extraversion. Journal of Personality and Social Psychology, 48(5), 1316-1327. doi:https://doi.org/10.1037/0022-3514.48.5.1316

Nostro, A. D., Müller, V. I., Varikuti, D. P., Pläschke, R. N., Hoffstaedter, F., Langner, R., ... Eickhoff, S. B. (2018). Predicting personality from network-based resting-state functional connectivity. Brain Structure and Function, 223(6), 2699-2719. doi:https://doi.org/10.1007/s00429-018-1651-z

Oldfield, R. C. (1971). The assessment and analysis of handedness: The Edinburgh inventory. Neuropsychologia, 9(1), 97-113. doi:https://doi.org/10.1016/0028-3932(71)90067-4

Pardo, Y., Aguilar, R., Molinuevo, B., \& Torrubia, R. (2007). Alcohol use as a behavioural sign of disinhibition: evidence from J.A. Gray's model of personality. Addictive Behaviors, 32(10), 2398-2403. doi:https://doi.org/10.1016/j.addbeh.2007.02.010

Pastor, M. C., Ross, S. R., Segarra, P., Montañés, S., Poy, R., \& Moltó, J. (2007). Behavioral inhibition and activation dimensions: Relationship to MMPI-2 indices of personality disorder. $\begin{array}{llll}\text { Personality and Individual } & \text { Differences, }\end{array}$ doi:https://doi.org/10.1016/J.PAID.2006.06.015 
Pickering, A. D., \& Gray, J. A. (1999). The neuroscience of personality. In L. A. Pervin \& O. P. John (Eds.), Handbook of personality: Theory and research (2nd edition) (pp. 277-299). New York: Guilford Press.

Pickering, A. D., \& Gray, J. A. (2001). Dopamine, appetitive reinforcement, and the neuropsychology of human learning: An individual differences approach. In A. Elias \& A. Angleitner (Eds.), Advances in individual differences research (pp. 113-149). Lengerich: PABST Science Publishers.

Pinto-Meza, A., Caseras, X., Soler, J., Puigdemont, D., Pérez, V., \& Torrubia, R. (2006). Behavioural inhibition and behavioural activation systems in current and recovered major depression participants. Personality and Individual Differences, 40(2), 215-226. doi:https://doi.org/10.1016/j.paid.2005.06.021

Power, J. D., Barnes, K. A., Snyder, A. Z., Schlaggar, B. L., \& Petersen, S. E. (2012). Spurious but systematic correlations in functional connectivity MRI networks arise from subject motion. NeuroImage, 59(3), 2142-2154. doi:https://doi.org/10.1016/J.NEUROIMAGE.2011.10.018

Pujara, M. S., Philippi, C. L., Motzkin, J. C., Baskaya, M. K., \& Koenigs, M. (2016). Ventromedial prefrontal cortex damage is associated with decreased ventral striatum volume and response to reward. The Journal of Neuroscience, 36(18), 5047-5054. doi:https://doi.org/10.1523/JNEUROSCI.4236-15.2016

Rangel, A., Camerer, C., \& Montague, P. R. (2008). A framework for studying the neurobiology of value-based decision making. Nature Reviews. Neuroscience, 9(7), 545-556. doi:https://doi.org/10.1038/nrn2357

Richard, J. M., \& Berridge, K. C. (2013). Prefrontal cortex modulates desire and dread generated by nucleus accumbens glutamate disruption. Biological Psychiatry, 73(4), 360-370. doi:https://doi.org/10.1016/J.BIOPSYCH.2012.08.009

Richter, A., \& Gruber, O. (2018). Influence of ventral tegmental area input on cortico-subcortical networks underlying action control and decision making. Human Brain Mapping, 39(2), 10041014. doi:https://doi.org/10.1002/hbm.23899

Rushworth, M. F. S., Noonan, M. P., Boorman, E. D., Walton, M. E., \& Behrens, T. E. (2011). Frontal cortex and reward-guided learning and decision-making. Neuron, 70(6), 1054-1069. doi:https://doi.org/10.1016/J.NEURON.2011.05.014

Sesack, S. R., Carr, D. B., Omelchenko, N., \& Pinto, A. (2003). Anatomical substrates for glutamate-dopamine interactions: Evidence for specificity of connections and extrasynaptic 
actions. Annals of the New York Academy of Sciences, 1003, 36-52. doi:https://doi.org/10.1196/annals.1300.066

Sescousse, G., Caldú, X., Segura, B., \& Dreher, J.-C. (2013). Processing of primary and secondary rewards: A quantitative meta-analysis and review of human functional neuroimaging studies. Neuroscience and Biobehavioral Reviews, 37(4), 681-696. doi:https://doi.org/10.1016/j.neubiorev.2013.02.002

Simon, J. J., Walther, S., Fiebach, C. J., Friederich, H.-C., Stippich, C., Weisbrod, M., \& Kaiser, S. (2010). Neural reward processing is modulated by approach- and avoidance-related personality $\begin{array}{llr}\text { traits. } & \text { NeuroImage, } & \text { 49(2), }\end{array}$ doi:https://doi.org/10.1016/J.NEUROIMAGE.2009.09.016

Smith, K. S., \& Graybiel, A. M. (2013). A dual operator view of habitual behavior reflecting cortical and striatal dynamics. Neuron, 79(2), 361-374. doi:https://doi.org/10.1016/J.NEURON.2013.05.038

Taylor, J., Reeves, M., James, L., \& Bobadilla, L. (2006). Disinhibitory trait profile and its relation to Cluster B personality disorder features and substance use problems. European Journal of Personality, 20(4), 271-284. doi:https://doi.org/10.1002/per.585

Torrubia, R., Avila, C., \& Caseras, X. (2008). Reinforcement sensitivity scales. In P. J. Corr (Ed.), The reinforcement sensitivity theory of personality (pp. 188-226). New York: Cambridge University Press.

Torrubia, R., Ávila, C., Moltó, J., \& Caseras, X. (2001). The Sensitivity to Punishment and Sensitivity to Reward Questionnaire (SPSRQ) as a measure of Gray's anxiety and impulsivity dimensions. Personality and Individual Differences, 31(6), 837-862. doi:https://doi.org/10.1016/S0191-8869(00)00183-5

Tziortzi, A. C., Haber, S. N., Searle, G. E., Tsoumpas, C., Long, C. J., Shotbolt, P., ... Gunn, R. N. (2014). Connectivity-Based Functional Analysis of Dopamine Release in the Striatum Using Diffusion-Weighted MRI and Positron Emission Tomography. Cerebral Cortex, 24(5), 11651177. doi:https://doi.org/10.1093/cercor/bhs397

Tzourio-Mazoyer, N., Landeau, B., Papathanassiou, D., Crivello, F., Etard, O., Delcroix, N., ... Joliot, M. (2002). Automated anatomical labeling of activations in SPM Using a macroscopic anatomical parcellation of the MNI MRI single-subject brain. NeuroImage, 15(1), 273-289. doi:https://doi.org/10.1006/nimg.2001.0978 
Vassena, E., Krebs, R. M., Silvetti, M., Fias, W., \& Verguts, T. (2014). Dissociating contributions of ACC and vmPFC in reward prediction, outcome, and choice. Neuropsychologia, 59, 112-123. doi:https://doi.org/10.1016/J.NEUROPSYCHOLOGIA.2014.04.019

Yan, C.-G., Craddock, R. C., He, Y., \& Milham, M. P. (2013). Addressing head motion dependencies for small-world topologies in functional connectomics. Frontiers in Human Neuroscience, 7, 910. doi:https://doi.org/10.3389/fnhum.2013.00910

Yan, C.-G., Wang, X.-D., Zuo, X.-N., \& Zang, Y.-F. (2016). DPABI: Data processing \& analysis for (Resting-State) brain imaging. Neuroinformatics, 14(3), 339-351. doi:https://doi.org/10.1007/s12021-016-9299-4

Yen, J.-Y., Cheng-Fang, Y., Chen, C.-S., Chang, Y.-H., Yeh, Y.-C., \& Ko, C.-H. (2012). The bidirectional interactions between addiction, behaviour approach and behaviour inhibition systems among adolescents in a prospective study. Psychiatry Research, 200(2-3), 588-592. doi:https://doi.org/10.1016/J.PSYCHRES.2012.03.015 


\title{
Reward network connectivity "at rest" is associated with reward sensitivity in healthy adults: a resting-state fMRI study
}

\author{
Supplementary Material
}

\section{Supplementary Methods}

\section{Control analysis}

Based on the different sizes of the ROIs selected as seeds, mainly between cortical and subcortical structures, one may argue that signal-to-noise levels could differ considerably across the ROIs, thus affecting the results. To rule out this possibility, we implemented a control analysis. Thus, we took spherical masks (5 mm radius) from the atlas provided by Power et al. (2011). Specifically, we selected the masks that were delimited within our a priori cortical ROIs (i.e. the ACC and the vmPFC) with an overlap of at least $80 \%$. As defined in the atlas, the spheres selected for the ACC were numbers 113 (MNI x, y, z = -3, 42, 16), $122(12,36,20)$, and $215(0,30,27)$, whereas for the vmPFC, they were $76(8,48,-15), 109(-3,44,-9)$, and $110(8,42,-5)$. All these ROIs are shown in Figure S1. Additionally, we also took two control ROIs not included in the reward network in order to study possible effects associated with high reward sensitivity (i.e. higher connectivity across the brain in general). To do so, we analyzed the connectivity between primary areas of the language network that are not expected to be related to SR. We defined these ROIs using spheres ( $5 \mathrm{~mm}$ radius) centered on the coordinates utilized by Tomasi \& Volkow (2012) for Broca's $(-51,27,18)$ and Wernicke's $(-51,-51,30)$ areas.

\section{Supplementary Results}

\section{Control analysis}

Results were consistent with our main analysis. Partial correlations (controlling for age and gender) revealed a significant association between SR scores and the rs-FC between the ACC_122-vmPFC_76 $(\mathrm{r}=.227, \mathrm{p}=.035)$, ACC_122-vmPFC_110 $(\mathrm{r}=.299, \mathrm{p}=.005)$, and ACC_215-vmPFC_76 $(\mathrm{r}=.213 \mathrm{p}=.048)$. Additionally, we observed a significant association between SR and the rs-FC between the vmPFC_109-VTA $(r=.250, p=.020)$ and vmPFC_110VTA $(r=.274, p=.010)$. These results provide evidence for the relationship between SR and functional connectivity across our a priori regions (i.e. the ROIs used for the main analysis) by ruling out effects associated with signal-to-noise ratio differences due to ROI size. Moreover, the replication of the results in diverse spheres within the ACC and the vmPFC suggest that the effects are better characterized by covering the whole regions. 
On the other hand, we did not observe any significant effects between SR and the rs-FC between Broca and Wernicke's areas $(r=.009, \mathrm{p}=.935)$, indicating that our effects are not driven by a global relationship between SR and brain rs-FC.
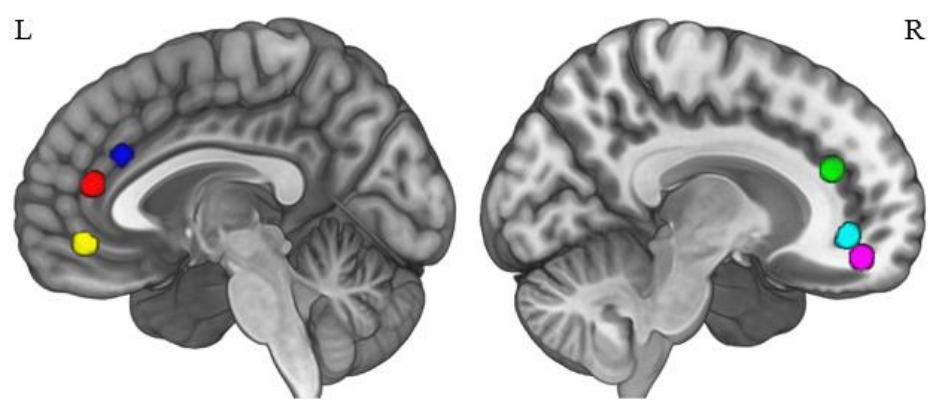

Figure S1. Seed ROIs selected from Power et al. (2011) delimited within the ACC and vmPFC ROIs. Blue: ACC_215; Red: ACC_113; Green: ACC_122; Yellow: vmPFC_109; Cyan: vmPFC_110; Purple: vmPFC_76.

\section{References}

Power, J. D., Cohen, A. L., Nelson, S. M., Wig, G. S., Barnes, K. A., Church, J. A., ... Petersen, S. E. (2011). Functional Network Organization of the Human Brain. Neuron, 72(4), 665-678. https://doi.org/10.1016/J.NEURON.2011.09.006

Tomasi, D., \& Volkow, N. D. (2012). Resting functional connectivity of language networks: characterization and reproducibility. Molecular Psychiatry, 17(8), 841-854. https://doi.org/10.1038/mp.2011.177 
Study 3

The manifestation of individual differences in sensitivity to punishment during resting state is modulated by eye state

Víctor Costumero, Jesús Adrián-Ventura, Elisenda Bueichekú, Anna Miró-Padilla, María-Ángeles Palomar-García, Lidón Marin-Marin, Esteban Villar-Rodríguez, Naiara Aguirre, Alfonso Barrós-Loscertales and César Ávila

Submitted 


\begin{abstract}
Neuroimaging studies have shown that brain areas associated with fear and anxiety are modulated by individual differences in sensitivity to punishment (SP). However, little is known about how SP relates to brain functional connectivity and the factors that modulate such a relationship. In this study, we investigate if a simple methodological manipulation such as performing a resting state with eyes open or eyes closed may modulate the manifestation of individual differences in SP. To this end, 88 participants performed a resting state with eyes closed and 56 with eyes open. All participants completed the Sensitivity to Punishment and Sensitivity to Reward Questionnaire. Seed-based functional connectivity analyses were performed in amygdala, hippocampus and periaqueductal gray (PAG). Our results showed that the relationship between SP and left amygdala-precuneus and left hippocampus-precuneus functional connectivity was modulated by eye state. Moreover, SP was positively related to the functional connectivity between left lateral PAG and amygdala in the eyes closed group, and negatively related to the functional connectivity of left lateral PAG and hippocampus in the eyes open group. Together, our results may suggest a different brain configuration during eyes open and eyes closed resting state, affecting the manifestation of individual differences in SP.
\end{abstract}

Keywords: sensitivity to punishment; anxiety; resting state; amygdala; hippocampus 


\section{Introduction}

Imagine a situation where something you are looking at is directly threating your body integrity. Punishment anticipation increases as the threat approaches, but, curiously, some people prefer to keep looking at what is actually happening (e.g., looking at the needle during a blood extraction), while others prefer to stop focusing on it just after evaluating the situation. Why individual differences in coping to threat exist is still an unresolved question, however, the study of the neural systems that promote those behaviors, and the factors that modulate these systems, may contribute to our understanding.

Fear and anxiety are adaptive emotions that engage responses to cope with actual and anticipated threat. The reinforcement sensitivity theory (RST) proposes that these emotions are mediated by two separated but interacting neurobiological systems involved in the defense of the organism (Gray \& McNaughton, 2000): the fight-flight-freeze system (FFFS) associated with fear, and the behavioral inhibition system (BIS) associated with anxiety. The FFFS mainly comprises the amygdala, medial hypothalamus and periaqueductal gray (PAG), and it is activated whenever the goal is to remove danger. The BIS is mainly composed by the septohippocampal system and amygdala, and it is activated when there are conflicting goals, such as when it is necessary to approach to a potential threat. The RST suggests that these defensive neural systems are modulated by individual differences in the intensity of the perceived threat, a dimension that is conceptualized as a personality trait of sensitivity to punishment (SP) (McNaughton \& Corr, 2004). Thus, under the same situation, individuals with higher SP would perceive, in general, threatening stimuli as more intense. Therefore, high SP individuals are more prone to experience fear, worry and rumination as well as to show avoidance and risk-assessment behaviors (Corr, 2004; McNaughton \& Corr, 2004). High SP has been proposed as a vulnerability factor for anxiety disorders (Gray \& McNaughton, 2000), and empirical studies have shown a positive relation between measures of this trait and anxiety disorders symptoms (Bijttebier, Beck, Claes, \& Vandereycken, 2009).

In recent years, neuroimaging studies have contributed to our understanding of the brain regions mediating individual differences in SP. For example, MRI structural studies have shown that high SP (or related traits) is associated with reduced volume and cortical thickness in medial prefrontal areas (Fuentes et al., 2012; Holmes et al., 2012) and a volume increase in the amygdala and hippocampus (Adrián-Ventura, Costumero, Parcet, \& Ávila, 2019; Barrós-Loscertales et al., 2006; Cherbuin et al., 2008; Holmes et al., 2012; Levita et al., 2014). Furthermore, functional MRI studies have shown that high SP (or related traits) is associated with increased activity of dorsal anterior cingulate cortex, posterior cingulate cortex, precuneus and amygdala, as well as reduced activity of ventral anterior cingulate cortex during negative events (Kennis, Rademaker, 
\& Geuze, 2013). However, little is known about how individual differences in SP relate to brain functional connectivity (FC). As far as we know, only one study has investigated FC individual differences in defensive system areas, using a specific scale to measure SP in the context of RST (Hahn et al., 2010). This study showed that the connectivity between hippocampus and amygdala during the processing of cues indicating potential loss was positively correlated with punishment sensitivity trait. Other studies have investigated individual differences in FC during resting state using scales related to SP such as neuroticism, harm avoidance or trait anxiety (Adelstein et al., 2011; Aghajani et al., 2014; Baeken et al., 2014; Gentili et al., 2017; Kim, Gee, Loucks, Davis, \& Whalen, 2011; Kruschwitz et al., 2014; Y. Li, Qin, Jiang, Zhang, \& Yu, 2012; Pang et al., 2016). However, although most of them coincide in showing individual differences in the FC of the amygdala, the connectivity patterns present little consistency across studies and in some cases are even contradictory. Therefore, further empirical evidence about the relationship between SP trait and individual differences in the FC of defensive system areas is necessary.

Beyond studying the association between SP and individual differences in FC, some studies have investigated the modulatory factors affecting these relationships. For example, it has been shown that men with low expression of X-linked Monoamine Oxidase A gene present specific negative FC between ventromedial prefrontal cortex and amygdala, which is in turn associated with individual differences in harm avoidance (Buckholtz et al., 2008). Furthermore, differences between the sexes have been shown in the correlations between harm avoidance and the FC patterns of laterobasal and centromedial subregions of the amygdala (Y. Li et al., 2012). In line with these previous studies, here we investigate whether a simple methodological manipulation, such as performing a resting state with eyes open (EO) or eyes closed (EC), may impact the manifestation of individual differences in the FC of defensive system areas. There is evidence showing that blood oxygen-level dependent (BOLD) signals of hippocampus and amygdala are modulated by EO and EC conditions (Jao et al., 2013; Liu, Dong, Zuo, Wang, \& Zang, 2013; Wiesmann et al., 2006). Moreover, a previous study investigating differences in FC between individuals with generalized anxiety disorder and controls showed that there were some differences that were irrespective of eye status, but also that some differences only manifested during a specific eye condition (W. Li et al., 2016). Notably, a previous EEG study showed how eye state modulated the relationship between frequency bands and diverse personality factors, including neuroticism (Konareva, 2011). Despite this evidence, no study has investigated whether or not eye state modulates the manifestation of personality factors in fMRI resting state paradigms.

The present study aims to investigate the effects of EO and EC conditions in the manifestation of SP related individual differences in the FC of defensive system areas. We hypothesize that we will show differences in the relationship between SP and the FC of defensive 
system areas according to EO and EC conditions. Accounting for the role of eye state during resting state, we attempt to provide new insights in the study of the neural basis of SP.

\section{Method}

\section{Participants}

One hundred and ninety-eight participants took part in this study (95 women; age: mean=22.2, $\mathrm{SD}=4.3$, range $=40-18$ ). Following subject exclusion due to excessive head motion (see preprocessing section), the final sample for analysis included one hundred and forty-four participants ( 75 women; age: mean=22.0, $\mathrm{SD}=4.0$, range $=40-18$ ). From the 144 participants, 56 performed an EO resting state session and 88 performed an EC resting state session. There were no differences in age $(\mathrm{t}=1.8 ; \mathrm{p}=0.07)$ and $\operatorname{sex}(\chi 2=0.6 ; \mathrm{p}=0.45)$ between the $\mathrm{EO}$ and $\mathrm{EC}$ groups. All the participants were right-handed according to the Edinburgh Handedness Inventory (Oldfield, 1971). No participant had a history of head injury with loss of consciousness, none currently used psychoactive medications, and none had been previously, or are currently, diagnosed with DSM-IV Axis I or II disorders or severe medical or neurological illnesses. Participants were informed of the nature of the research and provided written informed consent prior to their participation in the study. All the study procedures were approved by the Ethical Committee of the Jaume I University.

\section{Personality assessment}

The SP scale from the Sensitivity to Punishment and Sensitivity to Reward Questionnaire (SPSRQ) (Torrubia et al., 2001) was used as a measure of the punishment sensitivity trait (mean=9.7, $\mathrm{SD}=5.2$, range=23-0). No significant differences in punishment sensitivity scores were shown between the participants that performed the EC session and the participants that performed the EO session ( $\mathrm{t}=1.6 ; \mathrm{p}=0.12)$. The SP scale has good content validity and strongly correlates with other measures of punishment sensitivity, such as the Behavioral Inhibition Scale, Harm Avoidance, Punishment Expectancies and anxiety scales (Caseras, Àvila, Torrubia, Ávila, \& Torrubia, 2003).

\section{Image acquisition}

For the resting state EC sessions, participants were instructed to simply rest with their eyes closed and not to sleep or think about anything in particular. These same instructions, but with the specification of keeping the eyes open, were provided in EO sessions. Images were 
acquired on a $1.5 \mathrm{~T}$ scanner (Siemens Avanto; Erlanger, Germany). A total of 200 volumes were recorded using a gradient-echo T2*-weighted EPI sequence (TR, 2000 ms; TE, 48 ms; matrix, 64 x 64; voxel size, 3.5 x $3.5 \mathrm{~mm}$; flip angle, $90^{\circ}$; slice thickness, $4 \mathrm{~mm}$; slice gap, $0.8 \mathrm{~mm}$ ). We acquired 24 interleaved axial slices parallel to the anterior-posterior commissure plane covering the entire brain. Prior to the resting sequences, structural images were acquired using a T1weighted MPRAGE sequence with TR/TE $=2200 / 3.79 \mathrm{~ms}$, flip angle $15^{\circ}$, voxel size $=1 \times 1 \times 1$ $\mathrm{mm}$.

\section{Image preprocessing}

We used the DPABI toolbox (v2.1, http://rfmri.org/dpabi) (C.-G. Yan, Wang, Zuo, \& Zang, 2016) for image analyses. Preprocessing included the following steps: 1) removal of the first five volumes; 2) slice timing correction; 3) head motion correction using a six-parameter (rigid body) linear transformation; 4) co-registration of individual structural images with the mean functional image; 5) segmentation of structural images into grey matter, white matter and cerebrospinal fluid (CFS) using DARTEL (Ashburner, 2007); 6) removal of spurious variance through linear regression: 24 parameters from the head motion correction (Friston, Williams, Howard, Frackowiak, \& Turner, 1996), scrubbing within regression (C.-G. Yan et al., 2013) at framewise displacement of (FD)>0.2mm (Power, Barnes, Snyder, Schlaggar, \& Petersen, 2012), linear and quadratic trends, the white matter signal and the CFS signal; 7) spatial normalization to the MNI space (voxel size $3 \times 3 \times 3 \mathrm{~mm} 3$ ); 8) spatial smoothing with a $4 \mathrm{~mm}$ FWHM Gaussian Kernel; and 9) band-pass temporal filtering (0.01-0.1 Hz) (Biswal, Zerrin Yetkin, Haughton, \& Hyde, 1995; Lowe, Mock, \& Sorenson, 1998).

Participants with more than $1 \mathrm{~mm}$ or 1 degree of movement in any of the 6 directions, or less than 150 volumes with $\mathrm{FD}<0.2 \mathrm{~mm}$ (ensuring at least 5 minutes of rest with low FD), were excluded from the analyses.

\section{Functional connectivity analysis}

Seed-based correlation approach was performed to investigate how eye state modulates individual differences in the FC of the defensive system. We defined regions of interest (ROIs) for three key regions of the defensive system proposed in the last update of RST (Gray \& McNaughton, 2000); the periaqueductal gray, the amygdala and the hippocampus. For all ROIs, separate left and right seeds were defined. Furthermore, the periaqueductal gray was further subdivided in its ventrolateral portion (vlPAG) and its lateral portion (IPAG), based on recent revisions of RST suggesting a differential role on anxiety and fear, respectively (McNaughton \& 
Corr, 2008). All seeds were defined using the PickAtlas toolbox (Maldjian, Laurienti, Kraft, \& Burdette, 2003). The seeds for the amygdala and hippocampus were defined by the anatomical automatic labeling (AAL) template (Tzourio-Mazoyer et al., 2002); the seeds for vlPAG $( \pm 4,-26$, $-14 \mathrm{MNI}$ space $)$ and $\mathrm{PAG}( \pm 3,-28,-10 \mathrm{MNI}$ space $)$ were defined as 2-mm-radius spheres based on previous studies (Z. Chen et al., 2017; Kong, Tu, Zyloney, \& Su, 2010; Yu et al., 2014). FC for each participant and seed was calculated by correlating seeds' time series with the time series of every other voxel in the brain. Furthermore, we also performed specific pairwise correlations between the time series of our seeds in order to directly investigate possible eye state modulation in the relationship of SP and FC between these regions. After correlations, Fisher's $\mathrm{r}$ to $\mathrm{z}$ transformation was performed to normalize correlation values.

Voxel-wise analyses were performed using SPM12 (https://www.fil.ion.ucl.ac.uk/spm/software/spm12/). In order to study differences between EC and EO groups in the association between SP and FC maps, a whole-brain interaction analysis was performed using GLM for each seed. The model included two regressors defining groups and two regressors defining the SP scores (one per group). Age and sex were also included as nuisance regressors. The comparison between the regression slopes for SP regressors was the contrast of interest. Given the undirected nature of our hypothesis, we set a voxel level primary threshold of $\mathrm{p}<0.0005$ followed by a cluster level threshold of $\mathrm{p}<0.025$, in order to allow for a two-tailed FWE corrected threshold of $p<0.05$ (G. Chen et al., 2019). Significant voxel-wise results were further studied by post hoc tests using GLM. These analyses were restricted to a mask of the voxels showing interaction effect ( $\mathrm{p}<0.05 \mathrm{FWE}$ at voxel level). For the analysis regarding pairwise correlations between our seeds, we performed the same interaction model as described above in SPSS v23 (IBM Corp.). The statistical threshold for this analysis was set at $\mathrm{p}<0.05$ FDR-corrected (Benjamini \& Hochberg, 1995). For all significant interactions, we performed post hoc testing in order to further study the directionality of the results. This analysis consisted of Pearson correlations between FC and SP, for each group separately.

\section{Results}

Whole-brain voxel-wise analyses showed that SP-related individual differences in the FC maps of the amygdala and hippocampus were modulated by eye status. On one hand, we found a significant interaction effect in FC between the left amygdala and precuneus (MNI local maxima: $\mathrm{x}=-9, \mathrm{y}=-57, \mathrm{z}=51 ; \mathrm{t}(138)=4.66 ; \mathrm{k}=30)$. As shown in Fig. 1A, the EC group showed a positive relationship between SP and the FC of the left amygdala with the precuneus. In contrast, the EO group showed a negative relationship between these variables. This shift in the relationship between SP and FC was confirmed in post hoc testing, which showed voxels with a significant 
positive relationship between SP and FC in the EC group (MNI local maxima: $\mathrm{x}=-6, \mathrm{y}=-63, \mathrm{z}=57$; $\mathrm{t}(84)=4.83 ; \mathrm{k}=10)$ and voxels with a significant negative relationship between SP and FC in the EO group (MNI local maxima: $\mathrm{x}=-9, \mathrm{y}=-54, \mathrm{z}=48 ; \mathrm{t}(52)=4.76 ; \mathrm{k}=11$ ). Furthermore, we found a significant interaction in FC between the left hippocampus and precuneus (MNI local maxima: $\mathrm{x}=3, \mathrm{y}=-42, \mathrm{z}=54 ; \mathrm{t}(138)=5.36 ; \mathrm{k}=63)$. Similar to the results showed in the left amygdala, SP was positively related with the FC between the left hippocampus and precuneus in the EC group, but negatively related in the EO group (see Fig. 1B). Again, these results were confirmed in the post hoc test performed in the EC group (MNI local maxima: $\mathrm{x}=6, \mathrm{y}=-36, \mathrm{z}=51 ; \mathrm{t}(84)=4.14 ; \mathrm{k}=11$ ) and the EO group (MNI local maxima: $x=-3, y=-45, z=54 ; t(52)=4.61 ; k=29)$, separately.

A)

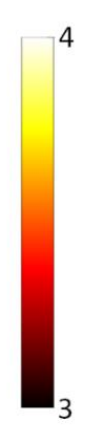

B)

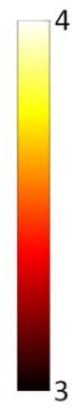

Left amygdala seed
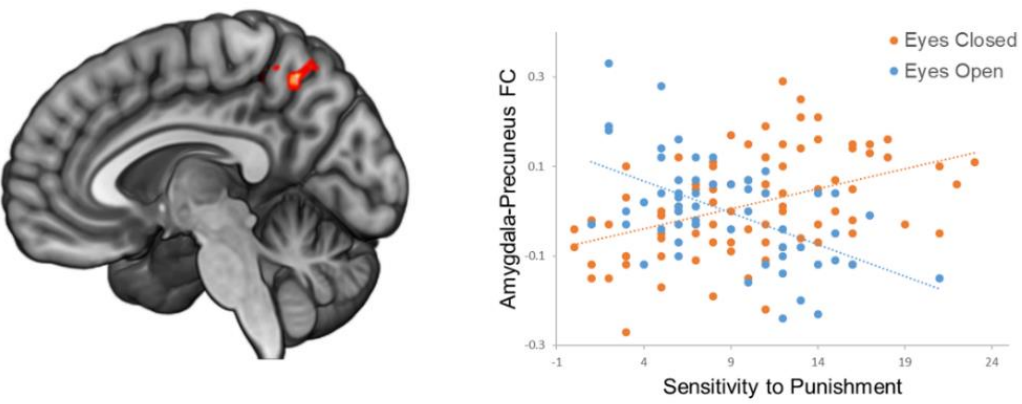

Left hippocampus seed

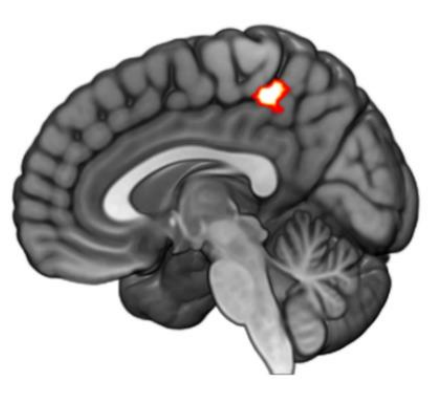

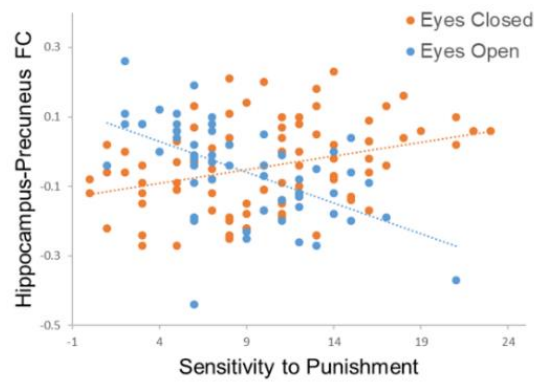

Fig. 1. Voxel-wise results. A) Left: brain map showing significant between-group differences in the relationship between sensitivity to punishment and individual differences in the functional connectivity of the amygdala. Right: scatter plot showing the relationship between sensitivity to punishment and functional connectivity for each group separately. The Y-axis represents the averaged functional connectivity values across the precuneus voxels showing significant between group differences in the functional connectivity with the amygdala. B) Left: brain map showing significant between-group differences in the relationship between sensitivity to punishment and individual differences in the functional connectivity of the hippocampus. Right: scatter plot showing the relationship between sensitivity to punishment and functional connectivity for each group separately. The Y-axis represents the averaged functional connectivity values across the precuneus voxels showing significant between group differences in the functional connectivity with the hippocampus. The color bars represent the t-value applicable to the images. Scatter plots are presented for illustrative purposes only. 
In addition to the whole-brain FC analyses, we directly investigated how eye state modulated individual differences in the FC between our seeds (see Fig. 2). These analyses revealed an interaction effect in the FC between the left amygdala and left IPAG ( $\mathrm{t}(138)=3.39$; $\mathrm{p}=0.01$ FDR corrected). Post hoc analyses revealed that the FC between these regions showed a significant positive correlation with SP in the EC group ( $r=0.33 ; \mathrm{p}=0.002)$, and no significant correlation in the EO group $(r=-0.24 ; p=0.08)$. Furthermore, we found a significant interaction in the FC between the left hippocampus and left lPAG $(t(138)=2.77 ; p=0.037$ FDR corrected). Post hoc analyses revealed that the FC between these regions showed a significant negative correlation with SP in the EO group ( $\mathrm{r}=-0.35 ; \mathrm{p}=0.009)$, and no correlation in the EC group $(\mathrm{r}=0.1 ; \mathrm{p}>0.1)$. Finally, for the specific relationship between SP and the FC between the amygdala and hippocampus reported in previous studies (Hahn et al., 2010), we found significant interaction effects only when we considered the uncorrected alpha level. These results were shown in the FC between the left hippocampus and left amygdala $(\mathrm{t}(138)=-2.2 ; \mathrm{p}=0.03$ uncorrected) as well as between the right hippocampus and right amygdala $(\mathrm{t}(138)=-2.46 ; \mathrm{p}=0.015$ uncorrected $)$. Post hoc analyses revealed that these interaction effects were driven by a positive relationship in the EO group (left hippocampus-left amygdala FC: $r=0.27 ; \mathrm{p}=0.046$; right hippocampus-right amygdala FC: $r=0.36 ; p=0.006$ ), with no relationship in the EC group ( $p>0.1)$. Fig. 3 shows a summary of all our results.
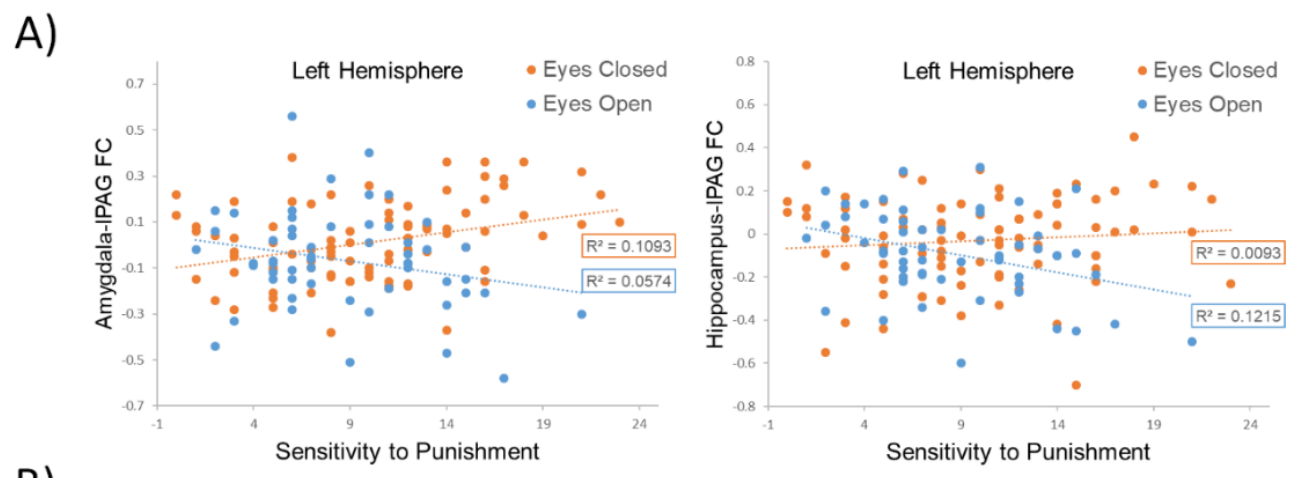

B)
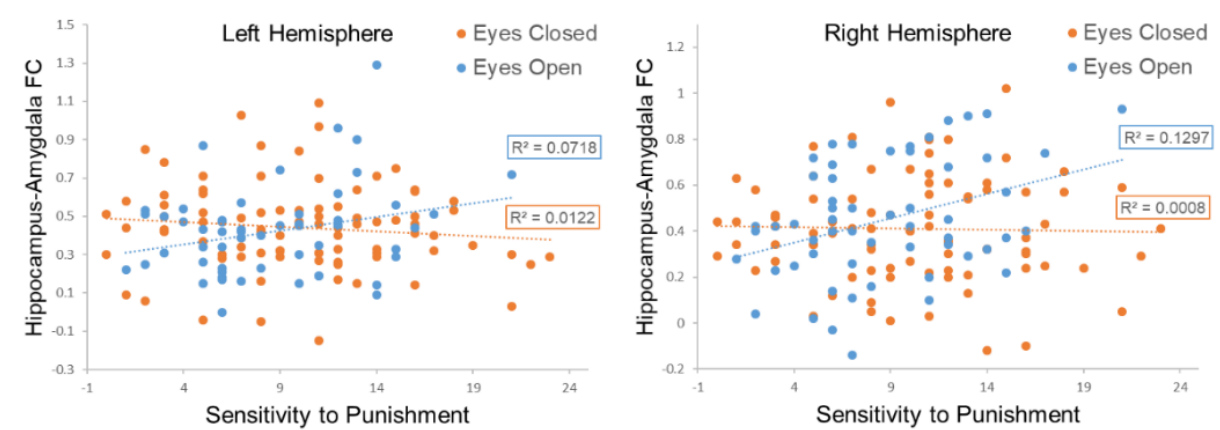

Fig. 2. Pairwise correlation results. A) Scatter plots showing the relationship between sensitivity to punishment and the functional connectivity between the left amygdala and left lateral periaqueductal gray (left panel) and between the left hippocampus and left lateral periaqueductal gray (right panel) for each group separately. B) Scatter plots showing the relationship between sensitivity to punishment and the functional connectivity between the amygdala and hippocampus in the left (left panel) and right (right panel) hemisphere for each group separately. 


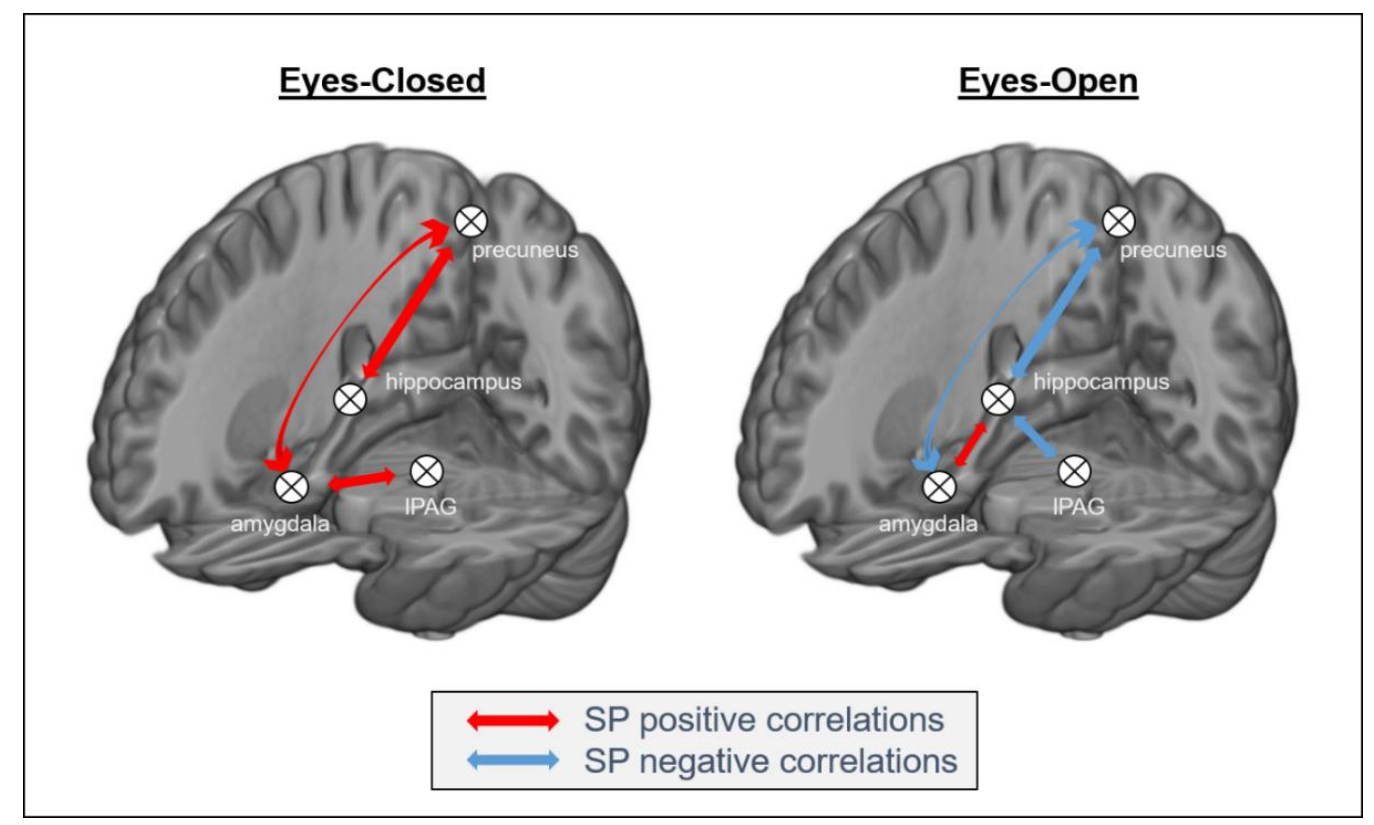

Fig. 3. Summary of the modulatory effects of eyes open and eyes closed conditions in the manifestation of functional connectivity individual differences associated with sensitivity to punishment. Red arrows show the brain regions which present a significant positive relationship between functional connectivity and sensitivity to punishment in the eyes closed group (left) and eyes open group (right) as determined by the post hoc tests. Blue arrows show the brain regions which present a significant negative relationship between functional connectivity and sensitivity to punishment in the eyes closed group (left) and eyes open group (right), as determined by the post hoc tests. The positive relationship between sensitivity to punishment and the functional connectivity between the hippocampus and amygdala during eyes open is included due to the previous findings of Hahn et al. (2010). In our study, this relationship was only significant when considering uncorrected alpha level.

\section{Discussion}

In this study we aimed to investigate how eye state at rest modulates the manifestation of individual differences in the FC of defensive system areas. To achieve this, we compared two groups of participants: an fMRI resting state scan was performed on each participant, with either EO or EC, and the SP scale from the SPSRQ was also completed for each. Whole-brain voxelwise analyses showed that eye state modulates the relationship between SP and individual differences in the FC of the left amygdala and left hippocampus with the precuneus. Furthermore, specific analyses performed to study the FC between our ROIs showed that left amygdala-left IPAG FC and left hippocampus-left IPAG FC were also modulated by eye state. These findings may suggest a differential brain FC configuration between EC and EO, which may influence the manifestation of individual differences in SP.

Evidence of modulatory effects of opening or closing eyes on brain activity has been shown using EEG since the beginning of the last century (Berger, 1929). In the field of fMRI, the evidence shows that the participants' eye state modulates brain activity and connectivity, not only 
in the visual cortex (Jao et al., 2013; Liu et al., 2013; Song et al., 2015; Wang, Li, Xu, \& Ding, 2015; Xu et al., 2014; C. Yan et al., 2009; D. Zhang et al., 2015). The specific causes explaining this modulatory effect are still unknown. However, there is evidence suggesting that it is an endogenous phenomenon, given that EO and EC modulatory effects have been shown independent of light input (Jao et al., 2013), even in early-blind individuals (Hüfner et al., 2009). Moreover, it has been suggested that these effects are associated with two different mental states: an "interoceptive" state, with the eyes closed, and an "exteroceptive" state, with the eyes open (Hüfner et al., 2009; Marx et al., 2004, 2003). The "exteroceptive" state would predominantly activate attentional and ocular motor systems, while the "interoceptive" state would be characterized by imagination and multisensory activity (Marx et al., 2003). Taking this model as reference, a straightforward explanation of our results may be drawn: a different brain activity configuration in EC and EO conditions might lead to a different manifestation of individual differences in SP within these conditions - especially taking into account the wide range of behaviors associated with SP - involving both "interoceptive" (e.g. worry, rumination) and "exteroceptive" (e.g. threat detection, scape/avoidance) states. However, it should be noted that our study is the first that specifically investigates the modulatory role of eye state in the relationship between FC and SP. Therefore, in the absence of additional empirical evidence this possibility is merely speculative.

In this study, whole-brain FC analyses showed a similar pattern of results in the left amygdala and left hippocampus seeds. With EC, the FC between the precuneus and these two regions was positively associated with SP. By contrast, SP was negatively associated with the FC between the precuneus and the two aforementioned regions during EO. The precuneus is functionally related to visuo-spatial imagery, episodic memory retrieval, self-processing and consciousness (Cavanna \& Trimble, 2006). Although the precuneus is widely known by its implication in the default mode network (Raichle et al., 2001), evidence from functional connectivity studies suggest that it could be subdivided in base to its connectivity patterns on its dorsal and ventral portions (S. Zhang \& Li, 2012). The ventral portion would be related to the default mode network, whereas the dorsal portion would be connected to areas associated with the dorsal attention network (Corbetta \& Shulman, 2002) - a brain network related to a variety of functions, including top-down control of visual attention, visuospatial imagery and working memory (Corbetta, Patel, \& Shulman, 2008; Mellet, Petit, Mazoyer, Denis, \& Tzourio, 1998; Ptak, Schnider, \& Fellrath, 2017; Tomasino \& Gremese, 2016). In our study, the region of the precuneus showing an interaction effect between eye state and SP in the connectivity with the amygdala and hippocampus was mainly located in the dorsal portion (Brodmann area 7). Therefore, it could be related to both "exteroceptive" and "interoceptive" behaviors. Individual differences in the connectivity between the amygdala and precuneus were previously shown in a 
study using the neuroticism scale from the NEO Five-Factor Inventory (Aghajani et al., 2014). Specifically, the authors of that study found that during an EC resting state session, the connectivity between the amygdala and dorsal precuneus was positively associated with individual differences in trait neuroticism. Although neuroticism and SP traits come from different theoretical models, they are conceptually related with negative emotion sensitivity and show a strong correlation in empirical studies (Aluja \& Blanch, 2011). Therefore, the positive relationship between SP and the connectivity between the amygdala and precuneus in the EC group shown in our study would agree with the results of this previous study.

When we analyzed seed-to-seed FC between our ROIs, we showed that eye state modulated individual differences in the left lPAG FC. Thus, during EC, the FC between the left IPAG and left amygdala was positively associated with SP, but during EO, the FC between the left IPAG and left hippocampus was negatively associated with SP. On one hand, the IPAG has been mainly related to fear and it is proposed that this mediates active defensive behaviors such as fight and flight (Fanselow, 1991; Linnman, Moulton, Barmettler, Becerra, \& Borsook, 2012). Regarding the modulatory effects of eye state, a previous study showed that the degree of FC between this region and the medial frontal cortex was associated with glutamate concentrations in the EC condition but not in the EO condition (Duncan et al., 2013). On the other hand, the latest revision of RST suggests that the amygdala is implicated in the control of active avoidance and the arousal associated with anxiety, while the hippocampus is suggested to be above in the hierarchy of the defensive system and mainly related to anxiety (McNaughton \& Corr, 2008). A previous fMRI study showed that hippocampus activity was increased after closing eyes in darkness (Wiesmann et al., 2006). Moreover, previous evidence showed that under an EC condition relative to an EO condition, amygdala BOLD signals show higher variance and regional homogeneity (Jao et al., 2013; Liu et al., 2013). In this study, we found differences in the relationship between SP and the FC between the amygdala and hippocampus as a function of eye state; however, these results were only significant if we considered the uncorrected threshold. Nonetheless, this result may be relevant because SP-related individual differences in the FC between the hippocampus and amygdala were shown in a previous fMRI study investigating brain response to punishment anticipation (Hahn et al., 2010). Specifically, in that study the authors showed, by means of beta series correlation method, that the connectivity between the hippocampus and amygdala during the presentation of visual cues signaling potential monetary loss was positively related with individual differences in SP. In our study, we also found a positive association between SP and the FC between the hippocampus and amygdala in the EO group, suggesting that individual differences in the connectivity between these regions are also present when eyes are open, but in the absence of punishment cues. Interestingly, Hahn and colleagues (2010) also performed a psychophysiological interaction analysis comparing punishment cues 
with neutral cues, but they did not find significant differences using this methodology. Psychophysiological interaction analysis is a method used to investigate specific differences in brain connectivity between task conditions. Given these negative results, and the results presented here, future studies should determine if the presence of aversive cues influences the relationship between SP and amygdala-hippocampus connectivity or if this relationship is actually driven by the existence of individual differences at rest.

Taking together our results, a different pattern of associations between FC and SP as a function of EC and EO conditions is suggested. As shown in Fig. 3, with an EC condition, the FC between hippocampus and amygdala seems to be unrelated with SP. However, the FC of these structures with other brain regions is more positive in participants with high SP. By contrast, in an EO condition, the FC between the hippocampus and amygdala is more positive in high SP individuals. However, the FC of these structures with other brain regions is more negative in these subjects. Future studies should confirm the existence of this specific pattern and establish the implications it may have for current neurobiological models of personality.

In summary, in this study we have shown that opening or closing eyes during resting state modulates the relationship between SP and individual differences in the FC of defensive system areas. Specifically, our results showed that precuneus-amygdala and precuneus-hippocampus FC was positively related to SP during EC but negatively during EO. Moreover, SP was positively related to the FC between the left IPAG and amygdala during EC, and negatively related with the FC of the left IPAG and hippocampus during EO. Together, our results may suggest a different brain configuration during EC and EO, which affects the manifestation of individual differences in SP.

\section{References}

Adelstein, J. S., Shehzad, Z., Mennes, M., DeYoung, C. G., Zuo, X.-N., Kelly, C., ... Milham, M. P. (2011). Personality Is Reflected in the Brain's Intrinsic Functional Architecture. PLoS One, 6(11), e27633. https://doi.org/10.1371/journal.pone.0027633

Adrián-Ventura, J., Costumero, V., Parcet, M. A., \& Ávila, C. (2019). Linking personality and brain anatomy: a structural MRI approach to Reinforcement Sensitivity Theory. Social Cognitive and Affective Neuroscience, 14(3), 329-338. https://doi.org/10.1093/scan/nsz011

Aghajani, M., Veer, I. M., van Tol, M.-J., Aleman, A., van Buchem, M. A., Veltman, D. J., ... van der Wee, N. J. (2014). Neuroticism and extraversion are associated with amygdala restingstate functional connectivity. Cognitive, Affective \& Behavioral Neuroscience, 14(2), 836-848. https://doi.org/10.3758/s13415-013-0224-0 
Aluja, A., \& Blanch, A. (2011). Neuropsychological Behavioral Inhibition System (BIS) and Behavioral Approach System (BAS) assessment: a shortened Sensitivity to Punishment and Sensitivity to Reward Questionnaire version (SPSRQ-20). Journal of Personality Assessment, 93(6), 628-636. https://doi.org/10.1080/00223891.2011.608760

Ashburner, J. (2007). A fast diffeomorphic image registration algorithm. NeuroImage, 38(1), 95113. https://doi.org/10.1016/J.NEUROIMAGE.2007.07.007

Baeken, C., Marinazzo, D., Van Schuerbeek, P., Wu, G. R., De Mey, J., Luypaert, R., \& De Raedt, R. (2014). Left and right amygdala - Mediofrontal cortical functional connectivity is differentially modulated by harm avoidance. PLoS One, 9(4), e95740. https://doi.org/10.1371/journal.pone.0095740

Barrós-Loscertales, A., Meseguer, V., Sanjuán, A., Belloch, V., Parcet, M. A., Torrubia, R., \& Avila, C. (2006). Behavioral Inhibition System activity is associated with increased amygdala and hippocampal gray matter volume: A voxel-based morphometry study. NeuroImage, 33(3), 10111015. https://doi.org/10.1016/j.neuroimage.2006.07.025

Benjamini, Y., \& Hochberg, Y. (1995). Controlling the False Discovery Rate: A Practical and Powerful Approach to Multiple Testing. Journal of the Royal Statistical Society. Series B (Methodological), 57, 289-300. https://doi.org/10.2307/2346101

Berger, H. (1929). Über das Elektrenkephalogramm des Menschen. Archiv Für Psychiatrie Und Nervenkrankheiten, 87(1), 527-570. https://doi.org/10.1007/BF01797193

Bijttebier, P., Beck, I., Claes, L., \& Vandereycken, W. (2009). Gray's Reinforcement Sensitivity Theory as a framework for research on personality-psychopathology associations. Clinical Psychology Review, 29(5), 421-430. https://doi.org/10.1016/j.cpr.2009.04.002

Biswal, B., Zerrin Yetkin, F., Haughton, V. M., \& Hyde, J. S. (1995). Functional connectivity in the motor cortex of resting human brain using echo-planar mri. Magnetic Resonance in Medicine, 34(4), 537-541. https://doi.org/10.1002/mrm.1910340409

Buckholtz, J. W., Callicott, J. H., Kolachana, B., Hariri, A. R., Goldberg, T. E., Genderson, M., ... Meyer-Lindenberg, A. (2008). Genetic variation in MAOA modulates ventromedial prefrontal circuitry mediating individual differences in human personality. Molecular Psychiatry, 13(3), 313-324. https://doi.org/10.1038/sj.mp.4002020

Caseras, X., Àvila, C., Torrubia, R., Ávila, C., \& Torrubia, R. (2003). The measurement of individual differences in Behavioural Inhibition and Behavioural Activation Systems: a comparison of personality scales. Personality and Individual Differences, 34(6), 999-1013. https://doi.org/10.1016/S0191-8869(02)00084-3 
Cavanna, A. E., \& Trimble, M. R. (2006). The precuneus: A review of its functional anatomy and behavioural correlates. Brain, 129(3), 564-583. https://doi.org/10.1093/brain/aw1004

Chen, G., Cox, R. W., Glen, D. R., Rajendra, J. K., Reynolds, R. C., \& Taylor, P. A. (2019). A tail of two sides: Artificially doubled false positive rates in neuroimaging due to the sidedness choice with t-tests. Human Brain Mapping, 40(3), 1037-1043. https://doi.org/10.1002/hbm.24399

Chen, Z., Chen, X., Liu, M., Liu, S., Ma, L., \& Yu, S. (2017). Disrupted functional connectivity of periaqueductal gray subregions in episodic migraine. The Journal of Headache and Pain, 18(1), 36. https://doi.org/10.1186/s10194-017-0747-9

Cherbuin, N., Windsor, T. D., Anstey, K. J., Maller, J. J., Meslin, C., \& Sachdev, P. S. (2008). Hippocampal volume is positively associated with behavioural inhibition (BIS) in a large community-based sample of mid-life adults: the PATH through life study. Social Cognitive and Affective Neuroscience, 3(3), 262-269. https://doi.org/10.1093/scan/nsn018

Corbetta, M., Patel, G., \& Shulman, G. L. (2008). The reorienting system of the human brain: from environment to theory of mind. Neuron, 58(3), 306-324. https://doi.org/10.1016/j.neuron.2008.04.017

Corbetta, M., \& Shulman, G. L. (2002). Control of goal-directed and stimulus-driven attention in the brain. Nature Reviews. Neuroscience, 3(3), 201-215. https://doi.org/10.1038/nrn755

Corr, P. J. (2004). Reinforcement sensitivity theory and personality. Neuroscience and Biobehavioral Reviews, 28(3), 317-332. https://doi.org/10.1016/j.neubiorev.2004.01.005

Duncan, N. W., Wiebking, C., Tiret, B., Marjańska, M., Hayes, D. J., Lyttleton, O., ... Northoff, G. (2013). Glutamate Concentration in the Medial Prefrontal Cortex Predicts Resting-State Cortical-Subcortical Functional Connectivity in Humans. PLoS One, 8(4), e60312. https://doi.org/10.1371/journal.pone.0060312

Fanselow, M. S. (1991). The Midbrain Periaqueductal Gray as a Coordinator of Action in Response to Fear and Anxiety. In A. Depaulis \& R. Bandler (Eds.), The Midbrain Periaqueductal Gray Matter (pp. 151-173). Boston: Springer. https://doi.org/10.1007/978-1-4615-3302-3_10

Friston, K. J., Williams, S., Howard, R., Frackowiak, R. S. J., \& Turner, R. (1996). MovementRelated effects in fMRI time-series. Magnetic Resonance in Medicine, 35(3), 346-355. https://doi.org/10.1002/mrm.1910350312

Fuentes, P., Barrós-Loscertales, A., Bustamante, J. C., Rosell, P., Costumero, V., \& Ávila, C. (2012). Individual differences in the Behavioral Inhibition System are associated with 
orbitofrontal cortex and precuneus gray matter volume. Cognitive, Affective \& Behavioral Neuroscience, 12(3), 491-498. https://doi.org/10.3758/s13415-012-0099-5

Gentili, C., Cristea, I. A., Ricciardi, E., Vanello, N., Popita, C., David, D., \& Pietrini, P. (2017). Not in one metric: Neuroticism modulates different resting state metrics within distinctive brain regions. Behavioural Brain Research, 327, 34-43. https://doi.org/10.1016/j.bbr.2017.03.031

Gray, J. A., \& McNaughton, N. (2000). The neuropsychology of anxiety: An inquiry into the functions of the septo-hippocampal system (2nd ed.). Oxford: Oxford University Press.

Hahn, T., Dresler, T., Plichta, M. M., Ehlis, A.-C., Ernst, L. H., Markulin, F., ... Fallgatter, A. J. (2010). Functional amygdala-hippocampus connectivity during anticipation of aversive events is associated with Gray's trait "sensitivity to punishment". Biological Psychiatry, 68(5), 459-464. https://doi.org/10.1016/j.biopsych.2010.04.033

Holmes, A. J., Lee, P. H., Hollinshead, M. O., Bakst, L., Roffman, J. L., Smoller, J. W., \& Buckner, R. L. (2012). Individual differences in amygdala-medial prefrontal anatomy link negative affect, impaired social functioning, and polygenic depression risk. The Journal of Neuroscience, 32(50), 18087-18100. https://doi.org/10.1523/JNEUROSCI.2531-12.2012

Hüfner, K., Stephan, T., Flanagin, V. L., Deutschländer, A., Stein, A., Kalla, R., ... Brandt, T. (2009). Differential effects of eyes open or closed in darkness on brain activation patterns in blind subjects. Neuroscience Letters, 466(1), 30-34. https://doi.org/10.1016/j.neulet.2009.09.010

Jao, T., Vértes, P. E., Alexander-Bloch, A. F., Tang, I.-N., Yu, Y.-C., Chen, J.-H., \& Bullmore, E. T. (2013). Volitional eyes opening perturbs brain dynamics and functional connectivity $\begin{array}{lllll}\text { regardless } & \text { of } & \text { input. } & \text { NeuroImage, }\end{array}$ https://doi.org/10.1016/j.neuroimage.2012.12.007

Kennis, M., Rademaker, A. R., \& Geuze, E. (2013). Neural correlates of personality: an integrative review. Neuroscience and Biobehavioral Reviews, 37(1), 73-95. https://doi.org/10.1016/j.neubiorev.2012.10.012

Kim, M. J., Gee, D. G., Loucks, R. A., Davis, F. C., \& Whalen, P. J. (2011). Anxiety dissociates dorsal and ventral medial prefrontal cortex functional connectivity with the amygdala at rest. Cerebral Cortex, 21(7), 1667-1673. https://doi.org/10.1093/cercor/bhq237

Konareva, I. N. (2011). Modulation of high-frequency EEG rhythms under conditions of the activation reaction: Dependence on psychological characteristics of personality. Neurophysiology, 43(1), 42-52. https://doi.org/10.1007/s11062-011-9184-6 
Kong, J., Tu, P. C., Zyloney, C., \& Su, T. P. (2010). Intrinsic functional connectivity of the periaqueductal gray, a resting fMRI study. Behavioural Brain Research, 211(2), 215-219. https://doi.org/10.1016/j.bbr.2010.03.042

Kruschwitz, J. D., Walter, M., Varikuti, D., Jensen, J., Plichta, M. M., Haddad, L., ... Walter, H. (2014). 5-HTTLPR/rs25531 polymorphism and neuroticism are linked by resting state functional connectivity of amygdala and fusiform gyrus. Brain Structure and Function, 220(4), 2373-2385. https://doi.org/10.1007/s00429-014-0782-0

Levita, L., Bois, C., Healey, A., Smyllie, E., Papakonstantinou, E., Hartley, T., \& Lever, C. (2014). The Behavioural Inhibition System, anxiety and hippocampal volume in a non-clinical population. Biology of Mood \& Anxiety Disorders, 4(1), 4. https://doi.org/10.1186/2045-5380-44

Li, W., Cui, H., Zhu, Z., Kong, L., Guo, Q., Zhu, Y., ... Li, C. (2016). Aberrant Functional Connectivity between the Amygdala and the Temporal Pole in Drug-Free Generalized Anxiety Disorder. Frontiers in Human Neuroscience, 10, 549. https://doi.org/10.3389/fnhum.2016.00549 Li, Y., Qin, W., Jiang, T., Zhang, Y., \& Yu, C. (2012). Sex-dependent correlations between the personality dimension of harm avoidance and the resting-state functional connectivity of amygdala subregions. PLoS One, 7(4), e35925. https://doi.org/10.1371/journal.pone.0035925

Linnman, C., Moulton, E. A., Barmettler, G., Becerra, L., \& Borsook, D. (2012). Neuroimaging of the periaqueductal gray: State of the field. NeuroImage, 60(1), 505-522. https://doi.org/10.1016/j.neuroimage.2011.11.095

Liu, D., Dong, Z., Zuo, X., Wang, J., \& Zang, Y. (2013). Eyes-open/eyes-closed dataset sharing for reproducibility evaluation of resting state fMRI data analysis methods. Neuroinformatics, 11(4), 469-476. https://doi.org/10.1007/s12021-013-9187-0

Lowe, M. J., Mock, B. J., \& Sorenson, J. A. (1998). Functional connectivity in single and multislice echoplanar imaging using resting state fluctuations. NeuroImage, 7(7), 119-132. https://doi.org/10.1006/nimg.1997.0315

Maldjian, J. A., Laurienti, P. J., Kraft, R. A., \& Burdette, J. H. (2003). An automated method for neuroanatomic and cytoarchitectonic atlas-based interrogation of fMRI data sets. NeuroImage, 19(3), 1233-1239. https://doi.org/10.1016/S1053-8119(03)00169-1

Marx, E., Deutschländer, A., Stephan, T., Dieterich, M., Wiesmann, M., \& Brandt, T. (2004). Eyes open and eyes closed as rest conditions: impact on brain activation patterns. NeuroImage, 21(4), 1818-1824. https://doi.org/10.1016/j.neuroimage.2003.12.026 
Marx, E., Stephan, T., Nolte, A., Deutschländer, A., Seelos, K. C., Dieterich, M., \& Brandt, T. (2003). Eye closure in darkness animates sensory systems. NeuroImage, 19(3), 924-934. https://doi.org/10.1016/s1053-8119(03)00150-2

McNaughton, N., \& Corr, P. J. (2004). A two-dimensional neuropsychology of defense: fear/anxiety and defensive distance. Neuroscience and Biobehavioral Reviews, 28(3), 285-305. https://doi.org/10.1016/j.neubiorev.2004.03.005

McNaughton, N., \& Corr, P. J. (2008). The neuropsychology of fear and anxiety: a foundation for Reinforcement Sensitivity Theory. In P. J. Corr (Ed.), The reinforcement sensitivity theory of personality (pp. 44-94). Cambridge: Cambridge University Press.

Mellet, E., Petit, L., Mazoyer, B., Denis, M., \& Tzourio, N. (1998). Reopening the mental imagery debate: lessons from functional anatomy. NeuroImage, 8(2), 129-139. https://doi.org/10.1006/nimg.1998.0355

Oldfield, R. C. C. (1971). The assessment and analysis of handedness: The Edinburgh inventory. Neuropsychologia, 9(1), 97-113. https://doi.org/10.1016/0028-3932(71)90067-4

Pang, Y., Cui, Q., Wang, Y., Chen, Y., Wang, X., Han, S., ... Chen, H. (2016). Extraversion and neuroticism related to the resting-state effective connectivity of amygdala. Scientific Reports, 6(1), 35484. https://doi.org/10.1038/srep35484

Power, J. D., Barnes, K. A., Snyder, A. Z., Schlaggar, B. L., \& Petersen, S. E. (2012). Spurious but systematic correlations in functional connectivity MRI networks arise from subject motion. NeuroImage, 59(3), 2142-2154. https://doi.org/10.1016/J.NEUROIMAGE.2011.10.018

Ptak, R., Schnider, A., \& Fellrath, J. (2017). The Dorsal Frontoparietal Network: A Core System for Emulated Action. Trends in Cognitive Sciences, 21(8), 589-599. https://doi.org/10.1016/j.tics.2017.05.002

Raichle, M. E., MacLeod, a M., Snyder, a Z., Powers, W. J., Gusnard, D. a, \& Shulman, G. L. (2001). A default mode of brain function. Proceedings of the National Academy of Sciences of the United States of America, 98(2), 676-682. https://doi.org/10.1073/pnas.98.2.676

Song, X., Zhou, S., Zhang, Y., Liu, Y., Zhu, H., \& Gao, J.-H. (2015). Frequency-Dependent Modulation of Regional Synchrony in the Human Brain by Eyes Open and Eyes Closed RestingStates. PLoS One, 10(11), e0141507. https://doi.org/10.1371/journal.pone.0141507

Tomasino, B., \& Gremese, M. (2016). Effects of Stimulus Type and Strategy on Mental Rotation Network: An Activation Likelihood Estimation Meta-Analysis. Frontiers in Human Neuroscience, 9, 693. https://doi.org/10.3389/fnhum.2015.00693 
Torrubia, R., Ávila, C., Moltó, J., \& Caseras, X. (2001). The Sensitivity to Punishment and Sensitivity to Reward Questionnaire (SPSRQ) as a measure of Gray's anxiety and impulsivity dimensions. Personality and Individual Differences, 31(6), 837-862. https://doi.org/10.1016/S0191-8869(00)00183-5

Tzourio-Mazoyer, N., Landeau, B., Papathanassiou, D., Crivello, F., Etard, O., Delcroix, N., ... Joliot, M. (2002). Automated Anatomical Labeling of Activations in SPM Using a Macroscopic Anatomical Parcellation of the MNI MRI Single-Subject Brain. NeuroImage, 15(1), 273-289. https://doi.org/10.1006/nimg.2001.0978

Wang, X.-H., Li, L., Xu, T., \& Ding, Z. (2015). Investigating the Temporal Patterns within and between Intrinsic Connectivity Networks under Eyes-Open and Eyes-Closed Resting States: A Dynamical Functional Connectivity Study Based on Phase Synchronization. PLoS One, 10(10), e0140300. https://doi.org/10.1371/journal.pone.0140300

Wiesmann, M., Kopietz, R., Albrecht, J., Linn, J., Reime, U., Kara, E., ... Stephan, T. (2006). Eye closure in darkness animates olfactory and gustatory cortical areas. NeuroImage, 32(1), 293300. https://doi.org/10.1016/j.neuroimage.2006.03.022

Xu, P., Huang, R., Wang, J., Van Dam, N. T., Xie, T., Dong, Z., ... Luo, Y. (2014). Different topological organization of human brain functional networks with eyes open versus eyes closed. NeuroImage, 90, 246-255. https://doi.org/10.1016/j.neuroimage.2013.12.060

Yan, C.-G., Cheung, B., Kelly, C., Colcombe, S., Craddock, R. C., Di Martino, A., ... Milham, M. P. (2013). A comprehensive assessment of regional variation in the impact of head micromovements on functional connectomics. NeuroImage, 76, 183-201. https://doi.org/10.1016/J.NEUROIMAGE.2013.03.004

Yan, C.-G., Wang, X.-D., Zuo, X.-N., \& Zang, Y.-F. (2016). DPABI: Data Processing \& Analysis for (Resting-State) Brain Imaging. Neuroinformatics, 14(3), 339-351. https://doi.org/10.1007/s12021-016-9299-4

Yan, C., Liu, D., He, Y., Zou, Q., Zhu, C., Zuo, X., ... Zang, Y. (2009). Spontaneous brain activity in the default mode network is sensitive to different resting-state conditions with limited cognitive load. PLoS One, 4(5), e5743. https://doi.org/10.1371/journal.pone.0005743

Yu, R., Gollub, R. L., Spaeth, R., Napadow, V., Wasan, A., \& Kong, J. (2014). Disrupted functional connectivity of the periaqueductal gray in chronic low back pain. NeuroImage: Clinical, 6, 100-108. https://doi.org/10.1016/j.nicl.2014.08.019 
Zhang, D., Liang, B., Wu, X., Wang, Z., Xu, P., Chang, S., ... Huang, R. (2015). Directionality of large-scale resting-state brain networks during eyes open and eyes closed conditions. Frontiers in Human Neuroscience, 9, 81. https://doi.org/10.3389/fnhum.2015.00081

Zhang, S., \& Li, C. S. (2012). Functional connectivity mapping of the human precuneus by resting state fMRI. NeuroImage, 59(4), 3548-3562. https://doi.org/10.1016/j.neuroimage.2011.11.023

\section{Acknowledgements}

This work was supported by grants from Generalitat Valenciana (PROMETEO/2017/109) and Ministerio de Economía y Competitividad (PSI2016-78805-R) to C.A. Also, it was supported by a grant from Jaume I University (UJI-B2016-21) to A.B-L. Additionally, this work was supported by a pre-doctoral graduate program grant (FPI-UJI to A.MP and National FPU to J.A-V, L.M-M, E.V-R and N.A) and a post-doctoral graduate program grant (Juan de la Cierva to V.C and postdoc-UJI to M-Á.P-G). Author E.B was funded by a postdoctoral grant from the "Generalitat Valenciana (2018 APOSTD)" and the "European Social Fund (Investing in your future)". 


\section{CHAPTER 3. DISCUSSION}

The main objective of this thesis was to provide new evidence that allows better comprehension of RST in humans, given that the foundations of this personality framework were established mostly based on animal experimentation, and less research has been dedicated to examining its correspondence in humans. Furthermore, most of the studies conducted so far have focused on the brain's differential response to rewards and punishments as a function of personality, whereas only a few investigations have analysed the relationship between personality traits and stable brain patterns (i.e. in brain structure and resting-state activity). In other words, although previous studies have examined how individuals' responses vary when facing emotional stimuli (i.e. by showing different tendencies in approach or avoidance behaviours), the way the brain works on a regular basis and its relationship with personality traits has been largely unexplored in personality neuroscience. By analysing this relationship, we can better understand the mechanisms that lead to a differential response in the presence of rewarding or aversive stimuli. For this purpose, we carried out three independent studies aimed at testing the neural correlates described in RST for the BAS, BIS, and FFFS, by means of MRI. The results from each study are discussed below.

In the first study, we analysed the brain structural correlates (e.g. in GMV) associated with the reward and punishment sensitivity dimensions. Individual differences in these traits (obtained via the SP and SR scales from the SPSRQ) were correlated with the whole-brain GMV and with the GMV of a priori ROIs (the NAcc and caudate for SR and the amygdala and hippocampus for SP) in a large sample consisting of 400 healthy young adults. On the one hand, our results replicated previous findings on SR and the GMV of the prefrontal cortex, whereas we also replicated previous studies by linking SP to the amygdala GMV. In relation to SR, the wholebrain analysis showed a negative correlation with the GMV of the MPFC and the left lateral prefrontal cortex (including dorsal, middle, and inferior regions). These results are consistent with a previous study presenting lower GMV in the prefrontal cortex in participants with high scores on SR (Barrós-Loscertales et al., 2006b) and with a study conducted in a large sample showing a negative correlation between CT and the scores obtained on a composite measure of NS (including the BAS scale of Carver and White; see Holmes et al., 2016). Regarding SP, ROI analysis revealed a positive correlation with the left amygdala GMV. This result coincides with a previous study relating a greater GMV in this area with SP (Barrós-Loscertales et al., 2006a). Likewise, Holmes et al. (2012) also reported, in a large sample, a positive relationship between the GMV of the amygdala and the scores obtained on a customised scale based on negative affect traits (including the BIS scale of Carver and White). On the other hand, contrary to our expectations, we did not find significant associations between SP and the hippocampus or 
between SR and the striatum. Thus, even though we found some anatomical correlates in line with RST (e.g. the prefrontal cortex and the amygdala), our results did not completely confirm our hypotheses. Interestingly, however, we observed a significant GMV reduction in the left accumbens and caudate nuclei in males with an overactive BAS (see Barrós-Loscertales et al., 2006a for similar results). Furthermore, the relationship between the NAcc volume and SR was found to be different between males and females. Hence, our results highlight the influence of sex on the association between SR and striatal volume.

Together, these results support RST by linking structural differences in frontostriatal and limbic areas with the SR and SP traits, respectively. Regarding the BAS, the GMV reduction observed in the lateral and medial prefrontal cortices aligns with previous studies showing anatomical differences in similar regions linked to the SR dimension (Barrós-Loscertales et al., 2006b; Holmes et al., 2016). These GMV and CT reductions have also been reported in studies measuring self-reported impulsivity traits (e.g. Impulsiveness from the TCI-R or the Barratt Impulsivity Scale (BIS-11); Korponay et al., 2017; Matsuo et al., 2009; Schilling et al., 2013). Additionally, this pattern has also been described in children and adolescents by measuring CT and impulsivity with the UPPS-P scale (Merz, He, \& Noble, 2018). In line with our results, these studies (see Korponay et al., 2017; Matsuo et al., 2009) also found a negative correlation between the BIS-11 questionnaire and the GMV in medial prefrontal regions. Moreover, the negative relationship between the striatal GMV and SR found in our study supports a previous study linking SR and striatal volume in males (Barrós-Loscertales et al., 2006b). A similar result was also obtained in a recent study using Impulsiveness from the TCI-R, where the ventral putamen GMV correlated negatively with the scores on this scale (Caravaggio et al., 2017). This volume reduction in the ventral striatum has also been associated with certain everyday life behaviours, for example, Facebook usage, with a higher effect in males (see Montag et al., 2017), and even with music reward sensitivity (Hernández et al., 2019).

In relation to SP, our results showed a positive correlation between the scores on this trait and the volume of the left amygdala. However, even though we replicated previous results (see Barrós-Loscertales et al., 2006b), we failed to extend these findings to the hippocampus, as suggested by prior research (see also Barrós-Loscertales et al., 2006a; Cherbuin et al., 2008; Levita et al., 2014). However, some methodological issues could account for these differences (e.g. sample sizes, number of males and females, age, MRI analyses techniques, among others). In fact, in a previous study carried out in a large sample, Holmes et al. (2012) reported a positive relationship between both the amygdala and hippocampus volumes and the scores obtained in a composite measure of negative affect, thus showing that both regions are associated with inhibited personalities. Moreover, the positive relationship between the amygdala GMV and anxiety- 
related traits has also been described using different measures (e.g. HA and Neuroticism; Iidaka et al., 2006; Koelsch et al., 2013; Kyeong et al., 2014).

All this anatomical evidence provides confirmation of the brain basis of the BAS and BIS/FFFS in humans by linking individual differences in SR and SP to stable brain patterns. Moreover, these volumetric correlates align with the behavioural repertoire associated with the overactivation of these systems. In relation to the BAS, different studies have associated a lower GMV in lateral prefrontal areas with a preference for immediate rewards (Bjork, Momenan, \& Hommer, 2009; Mohammadi et al., 2016; Wang et al., 2017). Therefore, these structural correlates (e.g. in the SFG, MFG, and IFG) are associated with diminished cognitive control and behavioural inhibition, which in turn may predispose individuals to exhibiting more impulsive and risky behaviours in the presence of reward cues. Similarly, the mPFC modulates rewarding goal-directed behaviours (Hayes et al., 2014; Hiser \& Koenigs, 2018). Indeed, this region is involved in subjective value-based decision-making (Acikalin, Gorgolewski, \& Poldrack, 2017; Bartra, McGuire, \& Kable, 2013; Clithero \& Rangel, 2014) and top-down (cortico-subcortical) emotion regulation processes (Etkin, Büchel, \& Gross, 2015; Etkin, Egner, \& Kalisch, 2011; PicóPérez, Radua, Steward, Menchón, \& Soriano-Mas, 2017; Ridderinkhof, Ullsperger, Crone, \& Nieuwenhuis, 2004). Hence, it seems plausible that these stable variances in the prefrontal cortex account for the individual differences associated with reward sensitivity: individuals who score high on this trait display deviant behaviours toward appetitive stimuli, as shown by a hyperactive sensation-seeking pattern aimed at detecting and approaching rewards (Ávila \& Torrubia, 2008). However, prefrontal alterations in key regions within the emotional control system may establish a different probability of engaging in reward-directed behaviours, thus leading to inter-subject variability. Together with the striatal reductions (more prominent in males), this anatomical pattern could underlie a psychopathological predisposition to externalising disorders (e.g. substance use and abuse, addictions, and ADHD) related to BAS activity (Bijttebier et al., 2009; Slobodskaya, 2016). Regarding the BIS/FFFS, the positive relationship found between the amygdala GMV and SP is consistent with previous studies showing a correlation between the GMV of this area and measurable fear- and anxiety-related behaviours. In this way, the amygdala has been considered a biomarker for inhibited/anxious personalities, even in childhood (Clauss et al., 2014; Qin et al., 2014). In the same vein, different studies have found a greater volume of this structure in generalised anxiety disorder patients (De Bellis et al., 2000; Etkin, Prater, Schatzberg, Menon, \& Greicius, 2009; Schienle, Ebner, \& Schäfer, 2011). Moreover, the amygdalar structure has also been related to genetic alleles in panic disorder (Smoller et al., 2014). All these results clearly establish a link between deviant anxious and avoidance behaviours (i.e. associated with higher BIS and FFFS activity) and the amygdala's GMV, which in turn may be considered a biological predisposition to internalising disorders (Bijttebier et al., 2009; Slobodskaya, 2016). 
In the second study, we investigated the functional connectivity at rest between key regions within the brain reward circuit - as defined based on a priori ROIs - and its association with the SR trait. To this end, we studied a sample consisting of 89 healthy young adults. First, we conducted a series of one-sample t-tests in order to observe the rsFC within the reward network. As expected, all the rsFCs were significant. Second, when analysing the association between personality and $\mathrm{rsFC}$, we observed a positive relationship between SR and the functional connectivity between the VTA and the vmPFC and between the vmPFC and the ACC. These results provide evidence for stable brain connectivity patterns linked to the personality trait of reward sensitivity.

It is relevant that this study confirmed the relationship between the BAS and the corticobasal ganglia circuit. The functional connectivity within this system (i.e. the connectivity between all the ROIs) clearly showed an interconnected circuit, with the exception of the VTA-vmPFC rsFC, which showed a very low coupling effect - indeed, the correlation between the two structures was negative. This result is consistent with the rsFC pattern found in Huckins et al. (2018), where the VTA was not functionally connected with frontal reward-related regions, suggesting that during a resting-state condition these areas would not be coupled. These results extend a previous report by Kahnt, Chang, Park, Heinzle, \& Haynes (2012), where in fact central regions of the OFC were negatively correlated with the midbrain. Furthermore, our results depicted a clear pattern: individual differences in SR modulate the brain connectivity within the reward system. Specifically, high SR positively correlates with the rsFC between the ACCvmPFC and between the VTA-vmPFC. This latter result may imply a greater activity of the mesocortical pathway in individuals with an overactive BAS. Importantly, this dopaminergic branch promotes motivated behaviours toward appetitive, rewarding stimuli (Arias-Carrión et al., 2010; D’Ardenne, McClure, Nystrom, \& Cohen, 2008; Haber \& Knutson, 2010). This pathway arises from the VTA, a small midbrain structure containing a large number of dopaminergic cells that target the vmPFC through WM fibre tracts. These cells are activated in the presence of novel and emotional stimuli, thus enhancing motivational value signals. Subsequently, these neurons project to the vmPFC (including the $\mathrm{mOFC}$ ), where these salient incentive stimuli are evaluated and contextually coded (Bromberg-Martin et al., 2010; Depue \& Collins, 1999; Hayes et al., 2014). Thus, the mesocortical dopaminergic pathway integrates a brain circuit involved in emotion-based decision-making subject to individual differences in personality.

The relationship between BAS-related personality traits, such as SR or extraversion, and the functioning of the brain reward system was previously described (Depue \& Collins, 1999; Pickering \& Gray, 2001) and confirmed by a number of task-based fMRI studies (see Kennis et al., 2013). However, in spite of the number of studies analysing this relationship through taskbased contingency paradigms, no previous studies have analysed this association - for example, 
by analysing the connectivity between the VTA and the vmPFC and its association with SR - in a resting-state condition. However, a previous study analysed the functional connectivity between the midbrain and the mOFC by comparing low- and high-magnitude reward incentive stimuli. Interestingly, the authors found that, during low-incentive stimuli, the two regions were more strongly connected in individuals with high SR (Costumero, Barrós-Loscertales, Bustamante, Ventura-Campos, Fuentes, \& Ávila, 2013). That is, in the presence of neutral, non-salient stimuli, the brain enters a checking mode aimed at seeking new rewarding stimuli in the environment, whereas the presence of appetitive stimuli would activate different brain areas (i.e. the ventral striatum) - thus reducing the connectivity between the VTA and vmPFC. In this way, individuals with an overactive BAS would exhibit greater ability (facilitation, see Depue \& Collins, 1999) in detecting rewarding stimuli, even in non-appetitive contexts - importantly, the resting-state condition (i.e. the brain's default activity) would mimic this state. Our study pinpoints this intersubject variability in the mesocortical pathway. Thus, individuals with greater approach motivation would be more prone to detecting salient stimuli and evaluate and code them as rewarding. This bias toward appetitive cues would produce faster and more vigorous responses once rewarding stimuli are present in the environment. In favour of this hypothesis, previous studies have found a positive association between BAS-related measures (the BAS scale, sensation-seeking, and exploratory excitability) and the neural activity of the primary mesocortical areas (the VTA and the vmPFC) in the presence of novel stimuli (Krebs, Schott, \& Düzel, 2009; Lawson et al., 2012) and during the omission of the rewards (Simon et al., 2010).

On the other hand, our study also showed novel results by linking SR with individual differences in the functional connectivity between the ACC and the vmPFC. At rest, these two regions were positively associated, but the SR trait was found to add variability to this connection. Specifically, we observed a positive correlation between SR scores and the ACC-vmPFC rsFC. These regions have classically been associated with reinforcement-guided actions; however, different fMRI studies have highlighted a functional dissociation between the two regions (Rushworth, Behrens, Rudebeck, \& Walton, 2007). Specifically, the vmPFC is responsible for valuate outcomes, thus adding subjective value signals to external stimuli and, therefore, modulating the value of choices. The ACC computes these signals and selects appropriate actions in order to value-maximise the rewards. In this way, the ACC monitors ongoing processes and adapts actions based on the expectation of rewards and punishments - or their absence (i.e. prediction error) (Clithero \& Rangel, 2014; Rangel \& Hare, 2010; Rushworth, Noonan, Boorman, Walton, \& Behrens, 2011; Vassena, Krebs, Silvetti, Fias, \& Verguts, 2014). Hence, the vmPFC and ACC form an integrated circuit aimed at optimising goal-directed decision-making based on the valuation of rewarding stimuli and the trade-off of efforts to obtain them (Bartra et al., 2013; Hogan, Galaro, \& Chib, 2019). 
The connectivity between the vmPFC and the ACC has also been shown to be essential for reward-related decision-making processes. Indeed, stronger connectivity between these structures has been associated with greater rewards when comparing higher- vs lower-value offers (Economides, Guitart-Masip, Kurth-Nelson, \& Dolan, 2014; Fatahi, Haghparast, Khani, \& Kermani, 2018). Even in the absence of stimuli, one may also expect these two structures to be functionally connected, given that the ACC signals rewarding events (and also reward prediction error) that are subsequently coded in the vmPFC. However, the vmPFC also codifies unexpected rewarding outcomes not previously predicted by the ACC. The previous finding linking the VTA and the vmPFC adds support to this hypothesis. Indeed, in line with our results, a recent study explored the functional connectivity between all these areas when pre-processing salient, nonrewarding (neutral) stimuli. In this study, Richter \& Gruber (2018) reported a noteworthy pattern: whereas the presence of rewarding stimuli co-activated the VTA and the ventral striatum, salience per se (non-rewarded) led to increased activity in the VTA and ventral striatum, as well as the OFC and ACC. Moreover, during the presence of these salient neutral stimuli, the VTA showed stronger functional connectivity with all these regions. This evidence supports the idea that, even in the absence of rewarding stimuli (as in a resting-sate condition), the brain is set to detect potential appetitive stimuli in the environment. Notably, this attentional mechanism would be abnormally triggered in individuals with higher BAS activity. Thus, approach behaviours in these individuals would be more frequent and vigorous in response to appetitive cues, due to an unbalanced dopaminergic signalling within the cortico-basal ganglia circuit. Individual differences in SR would serve as a potential trait marker of this deviant activity, which in turn could be associated with a proneness to develop externalising behaviours (Slobodskaya, 2016).

In the third study, we analysed the functional connectivity of the key regions within the defence system during a resting-state condition and its association with the personality trait of punishment sensitivity. To this end, we utilised two independent datasets: the first was obtained from a sample of 88 participants who performed the resting-state with their eyes closed (EC group), whereas the second was derived from a sample of 56 participants who performed the resting-state with their eyes open (EO group). Notably, we observed significant differences in the rsFC of the amygdala and hippocampus associated with SP. In brief, we found a significant coupling between the amygdala and the hippocampus and the precuneus linked to individual differences in SP. Likewise, the connectivity between the amygdala and hippocampus was also related to SP. These results are in line with previous investigations reporting a stronger rsFC between the amygdala-precuneus in high neuroticism individuals (Aghajani et al., 2014), as well as a higher connectivity between the amygdala-hippocampus in high SP individuals in the presence of aversive and neutral cues (Hahn et al., 2010). Moreover, our analyses also revealed a novel effect when analysing the PAG rsFC: both the amygdala and the hippocampus were 
functionally connected with the IPAG based on individual differences in SP trait. Furthermore, according to previous evidence highlighting the influence of the volitional eye state (i.e. EO or EC) on the BOLD signal of the amygdala and hippocampus (see Jao et al., 2013; Liu, Dong, Zuo, Wang, \& Zang, 2013; Wiesmann et al., 2006), we conducted a series of analyses to test whether the manifestation of individual differences in SP was modulated by the eye state. Thus, we analysed possible interaction effects between rsFC, personality, and eye state. Interestingly, some differences between the EO and EC groups emerged, thus postulating the influence of the eye state on the connectivity patterns observed as a function of personality. Therefore, the eye state would establish a different brain disposition related to "exteroceptive" (e.g. fight/flight/freeze) or "interoceptive" (e.g. worry/rumination) behaviours. All these results are detailed below.

The seed-based whole-brain voxel-wise analyses performed in this study revealed an interaction effect between SP and the rsFC of the amygdala, hippocampus, and precuneus when comparing groups of EC and EO during resting-state. For both the amygdala and hippocampus, the correlation with the precuneus as a function of SP was positive (stronger rsFC) in the EC group, whereas this correlation was negative (weaker rsFC) in the EO group. The precuneus has been related to a number of functions, such as mental imaginary, memory retrieval, and selfconsciousness (Cavanna, 2007; Cavanna \& Trimble, 2006). It is relevant that our findings were mainly located in the dorsal portion of the precuneus (Brodmann area 7) - that is, part of the dorsal attentional network. This network is involved in different cognitive functions, such as working memory, visuospatial imaginary, and top-down attentional control (voluntary orientation; see Corbetta, Patel, \& Shulman, 2008; Mellet, Petit, Mazoyer, Denis, \& Tzourio, 1998; Petersen \& Posner, 2012; Ptak, Schnider, \& Fellrath, 2017; Tomasino \& Gremese, 2016). The results obtained in the EC group are in line with previous research that used neuroticism as a measure of trait anxiety (Aghajani et al., 2014). Notably, in this study the authors reported a positive association between the amygdala-precuneus (Brodmann area 7) rsFC and scores on neuroticism. Additionally, the activity of these structures at rest has been consistently associated with trait neuroticism by using related metrics (Gentili et al., 2017). These results establish a clear pattern between the anxiety dimension and a differential engagement of punishment-related areas at rest. A greater communication between these regions would boost the detection and processing of aversive, threatening stimuli.

The seed-to-seed analyses also revealed significant interaction effects. Both the amygdala and the hippocampus showed a similar connectivity pattern in relation to the IPAG: the amygdalaIPAG and hippocampus-IPAG rsFCs were shown to be modulated by the eye state and individual differences in SP. However, only the amygdala-IPAG connectivity in the EC group and the hippocampus-IPAG connectivity in the EO group were significant. Likewise, when studying the rsFC between the amygdala and the hippocampus, significant effects emerged in both 
hemispheres. In addition, the connectivity pattern was identical: the correlation between the amygdala-hippocampus in the left and right hemispheres was only significant (positively) in the EO groups. The results obtained in this series of analyses display an interconnected circuit modulated by SP, whereas the direction of these connections is different based on the eye state. The direct and indirect paths between these three regions have been widely described in neuroanatomical studies examining fear- and anxiety-related responses (e.g. fight, flight and freeze; for reviews, see Gross \& Canteras, 2012; Linnman et al., 2012; McNaughton \& Corr, 2018; Mobbs \& Kim, 2015; Silva \& McNaughton, 2019; Tovote et al., 2015). The rRST proposes that these structures are integrated in a defensive system that works hierarchically: the hippocampus would be involved in higher-order conflict resolution (large distances, anxiety), the amygdala increases arousal levels and promotes active avoidance behaviours (intermediate distances), and the IPAG would be responsible for lower-order defensive responses (short distances, fear/panic; McNaughton \& Corr, 2004, 2008). Together, this circuit - modulated by the serotonin and noradrenergic systems - would be responsible for risk assessment and subsequent defensive behaviours (McNaughton \& Corr, 2018). As observed in this study, the connectivity between these regions interacts with the eye state and SP. Regarding the eye state, a previous study found that the connectivity between the PAG and cortical areas (mPFC) depends on glutamate concentrations in EC, but not in EO (Duncan et al., 2013). Regarding SP, our results are closely related to the defensive distance. Anxious individuals perceive defensive distances as shorter; that is, they perceive aversive stimuli as closer than they really are, which in turn leads to more frequent and intense defensive actions, even in the presence of inoffensive stimuli (McNaughton \& Corr, 2019). The stronger functional connectivity between these areas in high trait anxiety individuals would favour these behaviours even at rest, for example, by preparing the organism to detect potential aversive stimuli in the environment (i.e. by maintaining a hypervigilant state continuing over time).

On the other hand, our analyses also revealed a significant correlation between SP and the rsFC between the amygdala and hippocampus in the EO groups (for the left and right hemispheres). Interestingly, a previous study conducted with the same personality scale reported similar results. In this study, Hahn et al. (2010) found a positive correlation between the amygdala-hippocampus connectivity under a punishment-related paradigm (EO task-based). More specifically, the authors found that the coupling between these two regions in high SP individuals was greater during the presentation of visual cues signalling potential monetary loss. Furthermore, the authors confirmed, by means of psychophysiological interaction analysis, that this positive coupling was not driven by punishment cues per se, given that they did not find differences compared to neutral cues. This latter result is comparable to our findings: the higher functional connectivity between the amygdala-hippocampus would be observable not only in the 
presence of neutral cues, but even in the absence of stimuli. This higher connectivity in neurotic individuals, however, would facilitate posterior aversive Pavlovian learnings - that is, showing greater conditionability to fear (Tzschoppe et al., 2014).

All in all, the results from this study have direct implications for RST. We found a relationship between the amygdala/hippocampus and the precuneus in high SP individuals. These results add support to RST by linking the SP trait and the rsFC of the key limbic regions within the defence system - that is, the core structures of the BIS/FFFS - with cortical areas. Thus, anxious individuals (those with an overactive BIS/FFFS) would exhibit greater negative arousal levels accompanied by excessive worry/rumination. Likewise, the association between the amygdala/hippocampus and the IPAG, as well as the stronger connectivity between the former structures in high SP individuals would imply more frequent - due to shorter perceived defensive distances - and vigorous avoidance/escape behaviours. Therefore, all these results would draw a hyperactive defence system linked to internalising disorders in participants with higher BIS/FFFS activation levels (Slobodskaya, 2016).

\section{Conclusions}

Based on the aforementioned studies, the general conclusions drawn in this thesis are:

1. High SR scores (overactive BAS) are associated with a diminished GM volume in the prefrontal cortex (lateral and medial cortices) and with a GM volume reduction in the striatum (NAcc) in males. Thus, the SR trait reflects stable individual differences in the structural morphology of the dopaminergic frontostriatal system.

2. High SP scores (overactive BIS/FFFS) are linked to greater GM volume in the amygdala. Thus, the amygdala is postulated as the key area within the defence system, mediating defensive active behaviours in order to avoid potential threats.

3. The functional connectivity at rest between the VTA and the vmPFC is positively associated with SR. Hence, individuals with an overactive BAS (greater approach motivation) show a hyperconnected mesocortical system. The stronger rsFC between these areas might lead to biased, stable behavioural patterns focused on rapid detection and evaluation of possible rewarding stimuli in the environment.

4. The positive association between the ACC and vmPFC rsFC in high BAS activity individuals could serve to establish more frequent reward predictions in the presence of 
appetitive cues, as well as evaluating and contextually coding these outcomes as positive reinforcers.

5. At rest, the amygdala and the hippocampus are functionally coupled with the precuneus in individuals scoring high on SP (overactive BIS/FFFS). This enhanced connectivity links limbic areas involved in risk assessment and active avoidance with cortical regions (e.g. the precuneus) that modulate self-conscious and self-referential information. The stronger connectivity between all these regions could keep anxious behaviours stable over time. Additionally, the eye condition during the resting-state modulates the direction and strength of these connections.

6. At rest, the connectivity between the amygdala and the hippocampus and the IPAG is stronger in individuals with high SP. Likewise, the amygdala-hippocampus rsFC is stronger in these individuals. Together, anxious individuals would exhibit an interconnected defence circuit that mediates reactions to aversive stimuli. These individuals would show more frequent and energic defensive behaviours elicited by potential threats. Moreover, the direction of these correlations also varies as a function of the eye state during the resting-state. 


\section{CHAPTER 4. FUTURE LINES OF RESEARCH}

The results presented in this thesis provide new insights into the brain basis of RST. Applying cutting-edge MRI techniques, we obtained valuable results in relation to brain anatomy and functional connectivity at rest in large samples of healthy young adults. Additionally, we used a widely used questionnaire in personality psychology (e.g. the SPSRQ) as a measure of reward and punishment sensitivity. However, in spite of the relevance and usefulness of these results, new studies are needed to broaden RST as a framework to study personality and psychopathological predisposition.

In the first place, even though the SPSRQ and the BIS/BAS scales have been established as the "gold standard" for measuring BAS and BIS/FFFS activity in the past 20 years, recent investigations have proposed new measures to more accurately assess the activity of these systems. These new measures are: the Jackson-5 (Jackson, 2009), the Reinforcement Sensitivity Questionnaire (RSQ; Smederevac, Mitrović, Čolović, \& Nikolašević, 2014), the revised Reinforcement Sensitivity Theory Questionnaire (rRST-Q; Reuter, Cooper, Smillie, Markett, \& Montag, 2015), and the Reinforcement Sensitivity Theory of Personality Questionnaire (RSTPQ; Corr \& Cooper, 2016). Based on the rRST, all these questionnaires were aimed at developing independent measures for the BAS, BIS, and FFFS - due to the categorical separation between fear and anxiety (McNaughton \& Corr, 2004). Therefore, although the SP and BIS scales also provide a valid index for these dimensions - that is, Gray proposed the punishment sensitivity dimension as a measure of BIS and FFFS reactivity - evidence supports the distinction between the two dimensions (Perkins et al., 2007; Sylvers et al., 2011). Thus, these new questionnaires open up promising research lines that will provide a more in-depth understanding of the neurobiological systems depicted in RST.

Second, new MRI measures may provide additional confirmation of the brain correlates of RST. In particular, VBM results would be greatly complemented by surface-based metrics, such as cortical thickness or surface area. Indeed, although VBM metrics provide a good measure for computing cortical and subcortical volumes, these values stem from the interaction between cortical thickness and surface area (Winkler et al., 2018, 2010), whereas these latter measures provide more precise and specific cortical measurement. In this line, previous studies have also pointed out the genetic dissociation between cortical thickness and surface area (Panizzon et al., 2009; Winkler et al., 2010). Thus, surface-based metrics may offer new data that support previous anatomical evidence. On the other hand, a number of studies have previously investigated the relationship between brain activity and the personality traits derived from RST. These investigations have provided solid evidence about the functional correlates of the BAS and 
BIS/FFFS. However, the relationship between these systems and the functional activity of largescale networks (e.g. the visual, somatomotor, dorsal attentional, ventral attentional, limbic, frontoparietal, and default mode networks, see Yeo et al., 2011) has been explored much less. Thus, future studies should address this issue in order to obtain new results that extend previous evidence, so that we can relate networks from different cognitive domains to specific personality traits. This would provide further insights into the relationship between personality traits and cognitive processes (e.g. are the dorsal and ventral attentional networks associated with the SR trait?). In this line, graph theory approaches could also be implemented to obtain information about the connectivity properties between discrete nodes and networks and their association with personality.

Third, given the modulatory effect of different variables on brain anatomy and functional connectivity, new studies should be conducted to further understand these mechanisms. Based on previous evidence highlighting the influence of sex on the relationship between brain anatomy and personality (Nostro, Müller, Reid, \& Eickhoff, 2016), and the modulatory effect of the eye condition (e.g. EO or EC) on the manifestation of individual differences in the rsFC between anxiety-related areas (W. Li et al., 2016), we carried out a series of analyses with the aim of identifying these effects in our studies (see Study 1 and Study 3). Accordingly, some differences emerged in both studies, thus confirming the influence of these variables on the brain structure and function. However, new investigations would be needed to better describe these effects. Furthermore, a recent study demonstrated that rsFC patterns could differentially predict personality traits (as measured by the NEO-FFI) in males and females, thus pointing out sexdependent effects on rsFC associated with personality (Nostro et al., 2018). Therefore, sex should also be taken into account as a covariate of interest in future resting-state studies, given that by studying males and females together some underlying effects could be overshadowed.

Finally, it would be of great interest to explore possible interactions between the systems proposed in RST. That is, even though the main objective of MRI studies is to characterise the biological foundations of the BAS, BIS, and FFFS, interactions between these systems are expected, and so some neurobiological hallmarks should be redefined. This idea comes from the “joint subsystems hypothesis" (Corr, 2002; Pickering \& Corr, 2008; Smillie, Pickering, \& Jackson, 2006), which claims that approach behaviours (BAS-dependent) would be influenced by different levels of behavioural inhibition (BIS/FFFS-dependent) and vice versa. For example, high BAS activation + low BIS activation would foster more vigorous approach behaviours. Some previous evidence supports this hypothesis. In fact, a previous study successfully tested this hypothesis by reporting a significant interaction between SR+/SP- in terms of striatal activation (Mortensen, Lehn, Evensmoen, \& Håberg, 2015). Thus, new studies that consider these interaction effects would provide novel results that would increase behavioural predictions. 
References for general introduction and discussion

Abler, B., Walter, H., Erk, S., Kammerer, H., \& Spitzer, M. (2006). Prediction error as a linear function of reward probability is coded in human nucleus accumbens. NeuroImage, 31(2), 790-795. https://doi.org/10.1016/j.neuroimage.2006.01.001

Abrol, A., Damaraju, E., Miller, R. L., Stephen, J. M., Claus, E. D., Mayer, A. R., \& Calhoun, V. D. (2017). Replicability of time-varying connectivity patterns in large resting state fMRI samples. NeuroImage, 163, 160-176. https://doi.org/10.1016/j.neuroimage.2017.09.020

Acikalin, M. Y., Gorgolewski, K. J., \& Poldrack, R. A. (2017). A Coordinate-Based MetaAnalysis of Overlaps in Regional Specialization and Functional Connectivity across Subjective Value and Default Mode Networks. Frontiers in Neuroscience, 11, 1. https://doi.org/10.3389/fnins.2017.00001

Aghajani, M., Veer, I. M., van Tol, M.-J., Aleman, A., van Buchem, M. A., Veltman, D. J., ... van der Wee, N. J. (2014). Neuroticism and extraversion are associated with amygdala resting-state functional connectivity. Cognitive, Affective \& Behavioral Neuroscience, 14(2), 836-848. https://doi.org/10.3758/s13415-013-0224-0

Alloy, L. B., \& Abramson, L. Y. (2010). The role of the behavioral approach system (BAS) in bipolar spectrum disorders. Current Directions in Psychological Science, 19(3), 189-194. https://doi.org/10.1177/0963721410370292

Alloy, L. B., Bender, R. E., Whitehouse, W. G., Wagner, C. A., Liu, R. T., Grant, D. A., ... Abramson, L. Y. (2012). High behavioral approach system (BAS) sensitivity, reward responsiveness, and goal-striving predict first onset of bipolar spectrum disorders: A prospective behavioral high-risk design. Journal of Abnormal Psychology, 121(2), 339-351. https://doi.org/10.1037/a0025877

Aluja, A. (2004). Sensitivity to punishment, sensitivity to reward and sexuality in females. Personality and Individual Differences, 36(1), 5-10. https://doi.org/10.1016/S01918869(02)00219-2

Anderson, D. J., \& Adolphs, R. (2014). A framework for studying emotions across species. Cell, 157(1), 187-200. https://doi.org/10.1016/j.cell.2014.03.003

Angelides, N. H., Gupta, J., \& Vickery, T. J. (2017). Associating resting-state connectivity with trait impulsivity. Social Cognitive and Affective Neuroscience, 12(6), 1001-1008. https://doi.org/10.1093/scan/nsx031 
Arias-Carrión, O., Stamelou, M., Murillo-Rodríguez, E., Menéndez-González, M., \& Pöppel, E. (2010). Dopaminergic reward system: a short integrative review. International Archives of Medicine, 3, 24. https://doi.org/10.1186/1755-7682-3-24

Avila, C. (2001). Distinguishing BIS-mediated and BAS-mediated disinhibition mechanisms: a comparison of disinhibition models of Gray $(1981,1987)$ and of Patterson and Newman (1993). Journal of Personality and Social Psychology, 80(2), 311-324. https://doi.org/10.1037/0022-3514.80.2.311

Avila, C., Garbin, G., Sanjuán, A., Forn, C., Barrós-Loscertales, A., Bustamante, J. C., ... Parcet, M. A. (2012). Frontostriatal response to set switching is moderated by reward sensitivity. Social Cognitive and Affective Neuroscience, 7(4), 423-430. https://doi.org/10.1093/scan/nsr028

Avila, C., Moltó, J., \& Segarra, P. (1995). Sensitivity to Conditioned or Unconditioned Stimuli: What Is the Mechanism Underlying Passive Avoidance Deficits in Extraverts? Journal of Research in Personality, 29(4), 373-394. https://doi.org/10.1006/JRPE.1995.1022

Avila, C., \& Parcet, M. A. (2000). The role of Gray's impulsivity in anxiety-mediated differences in resistance to extinction. European Journal of Personality, 14(3), 185-198. https://doi.org/10.1002/1099-0984(200005/06)14:3<185::AID-PER370>3.0.CO;2-U

Ávila, C., \& Torrubia, R. (2004). Personality, expectations, and response strategies in multiplechoice question examinations in university students: a test of Gray's hypotheses. European Journal of Personality, 18(1), 45-59. https://doi.org/10.1002/per.506

Ávila, C., \& Torrubia, R. (2008). Performance and conditioning studies. In P. J. Corr (Ed.), The reinforcement sensitivity theory of personality (pp. 228-260). New York: Cambridge University Press.

Baeken, C., De Raedt, R., Ramsey, N., Van Schuerbeek, P., Hermes, D., Bossuyt, A., ... Luypaert, R. (2009). Amygdala responses to positively and negatively valenced baby faces in healthy female volunteers: Influences of individual differences in harm avoidance. Brain Research, 1296, 94-103. https://doi.org/10.1016/j.brainres.2009.08.010

Baeken, C., Marinazzo, D., Van Schuerbeek, P., Wu, G. R., De Mey, J., Luypaert, R., \& De Raedt, R. (2014). Left and right amygdala - Mediofrontal cortical functional connectivity is differentially modulated by harm avoidance. PLoS One, 9(4), e95740. https://doi.org/10.1371/journal.pone.0095740

Balconi, M., Finocchiaro, R., \& Campanella, S. (2014). Reward sensitivity, decisional bias, and metacognitive deficits in cocaine drug addiction. Journal of Addiction Medicine, 8(6), 399- 
406. https://doi.org/10.1097/ADM.0000000000000065

Ball, S. A., \& Zuckerman, M. (1990). Sensation seeking, Eysenck's personality dimensions and reinforcement sensitivity in concept formation. Personality and Individual Differences, 11(4), 343-353. https://doi.org/10.1016/0191-8869(90)90216-E

Barlow, D. H. (1988). Anxiety and its disorders: the nature and treatment of anxiety disorder. New York: Guilford.

Barrós-Loscertales, A., Meseguer, V., Sanjuán, A., Belloch, V., Parcet, M. A., Torrubia, R., \& Avila, C. (2006a). Behavioral Inhibition System activity is associated with increased amygdala and hippocampal gray matter volume: A voxel-based morphometry study. NeuroImage, 33(3), 1011-1015. https://doi.org/10.1016/j.neuroimage.2006.07.025

Barrós-Loscertales, A., Meseguer, V., Sanjuán, A., Belloch, V., Parcet, M. A., Torrubia, R., \& Avila, C. (2006b). Striatum gray matter reduction in males with an overactive behavioral activation system. The European Journal of Neuroscience, 24(7), 2071-2074. https://doi.org/10.1111/j.1460-9568.2006.05084.x

Barrós-Loscertales, A., Ventura-Campos, N., Sanjuán-Tomás, A., Belloch, V., Parcet, M. A., \& Avila, C. (2010). Behavioral activation system modulation on brain activation during appetitive and aversive stimulus processing. Social Cognitive and Affective Neuroscience, 5(1), 18-28. https://doi.org/10.1093/scan/nsq012

Bartra, O., McGuire, J. T., \& Kable, J. W. (2013). The valuation system: A coordinate-based meta-analysis of BOLD fMRI experiments examining neural correlates of subjective value. NeuroImage, 76, 412-427. https://doi.org/10.1016/J.NEUROIMAGE.2013.02.063

Beaver, J. D., Lawrence, A. D., van Ditzhuijzen, J., Davis, M. H., Woods, A., \& Calder, A. J. (2006). Individual differences in reward drive predict neural responses to images of food. The Journal of Neuroscience, 26(19), 5160-5166. https://doi.org/10.1523/JNEUROSCI.0350-06.2006

Beck, I., Smits, D. J. M., Claes, L., Vandereycken, W., \& Bijttebier, P. (2009). Psychometric evaluation of the behavioral inhibition/behavioral activation system scales and the sensitivity to punishment and sensitivity to reward questionnaire in a sample of eating disordered patients. Personality and Individual Differences, 47(5), 407-412. https://doi.org/10.1016/j.paid.2009.04.007

Berridge, K. C. (2007). The debate over dopamine's role in reward: the case for incentive salience. Psychopharmacology, 191(3), 391-431. https://doi.org/10.1007/s00213-006-0578-x

Berridge, K. C., \& Robinson, T. E. (1998). What is the role of dopamine in reward: hedonic 
impact, reward learning, or incentive salience? Brain Research Reviews, 28(3), 309-369. https://doi.org/10.1016/S0165-0173(98)00019-8

Berridge, K. C., Robinson, T. E., \& Aldridge, J. W. (2009). Dissecting components of reward: "liking", "wanting", and learning. Current Opinion in Pharmacology, 9(1), 65-73. https://doi.org/10.1016/j.coph.2008.12.014

Bijttebier, P., Beck, I., Claes, L., \& Vandereycken, W. (2009). Gray’s Reinforcement Sensitivity Theory as a framework for research on personality-psychopathology associations. Clinical Psychology Review, 29(5), 421-430. https://doi.org/10.1016/j.cpr.2009.04.002

Biswal, B., Zerrin Yetkin, F., Haughton, V. M., \& Hyde, J. S. (1995). Functional connectivity in the motor cortex of resting human brain using echo-planar mri. Magnetic Resonance in Medicine, 34(4), 537-541. https://doi.org/10.1002/mrm.1910340409

Bjork, J. M., Momenan, R., \& Hommer, D. W. (2009). Delay Discounting Correlates with Proportional Lateral Frontal Cortex Volumes. Biological Psychiatry, 65(8), 710-713. https://doi.org/10.1016/j.biopsych.2008.11.023

Björklund, A., \& Dunnett, S. B. (2007). Dopamine neuron systems in the brain: an update. Trends in Neurosciences, 30(5), 194-202. https://doi.org/10.1016/j.tins.2007.03.006

Bjørnebekk, A., Fjell, A. M., Walhovd, K. B., Grydeland, H., Torgersen, S., \& Westlye, L. T. (2013). Neuronal correlates of the five factor model (FFM) of human personality: Multimodal imaging in a large healthy sample. NeuroImage, 65, 194-208. https://doi.org/10.1016/j.neuroimage.2012.10.009

Blanchard, R. J., \& Blanchard, D. C. (1990a). An ethoexperimental analysis of defense, fear and anxiety. In N. McNaughton \& G. Andrews (Eds.), Anxiety (pp. 124-133). Dunedin: Otago University Press.

Blanchard, R. J., \& Blanchard, D. C. (1990b). Anti-predator defense as models of animal fear and anxiety. In P. F. Brain, S. Parmigiani, R. J. Blanchard, \& D. Mainardi (Eds.), Fear and Defence (pp. 89-108). Chur: Harwood Academic Publishers.

Boog, M., Goudriaan, A. E., Wetering, B. J. M. v. d., Polak, M., Deuss, H., \& Franken, I. H. A. (2014). Rash Impulsiveness and Reward Sensitivity as predictors of treatment outcome in male substance dependent patients. Addictive Behaviors, 39(11), 1670-1675. https://doi.org/10.1016/J.ADDBEH.2014.02.020

Bourdy, R., Sánchez-Catalán, M. J., Kaufling, J., Balcita-Pedicino, J. J., Freund-Mercier, M. J., Veinante, P., ... Barrot, M. (2014). Control of the nigrostriatal dopamine neuron activity and motor function by the tail of the ventral tegmental area. Neuropsychopharmacology, 
39(12), 2788-2798. https://doi.org/10.1038/npp.2014.129

Bradley, B. P., Mogg, K., Falla, S. J., \& Hamilton, L. R. (1998). Attentional Bias for Threatening Facial Expressions in Anxiety: Manipulation of Stimulus Duration. Cognition and Emotion, 12(6), 737-753. https://doi.org/10.1080/026999398379411

Broadbent, D. E., Broadbent, M. H. P., \& Jones, J. L. (1986). Performance correlates of selfreported cognitive failure and of obsessionality. British Journal of Clinical Psychology, 25(4), 285-299. https://doi.org/10.1111/j.2044-8260.1986.tb00708.x

Bromberg-Martin, E. S., Matsumoto, M., \& Hikosaka, O. (2010). Dopamine in Motivational Control: Rewarding, Aversive, and Alerting. Neuron, 68(5), 815-834. https://doi.org/10.1016/J.NEURON.2010.11.022

Buckholtz, J. W., Callicott, J. H., Kolachana, B., Hariri, A. R., Goldberg, T. E., Genderson, M., ... Meyer-Lindenberg, A. (2008). Genetic variation in MAOA modulates ventromedial prefrontal circuitry mediating individual differences in human personality. Molecular Psychiatry, 13(3), 313-324. https://doi.org/10.1038/sj.mp.4002020

Bunzeck, N., \& Düzel, E. (2006). Absolute Coding of Stimulus Novelty in the Human Substantia Nigra/VTA. Neuron, 51(3), 369-379. https://doi.org/10.1016/j.neuron.2006.06.021

Byrne, A., \& Eysenck, M. W. (1995). Trait Anxiety, Anxious Mood, and Threat Detection. Cognition and Emotion, 9(6), 549-562. https://doi.org/10.1080/02699939508408982

Canli, T., Zhao, Z., Desmond, J. E., Kang, E., Gross, J., \& Gabrieli, J. D. E. (2001). An fMRI study of personality influences on brain reactivity to emotional stimuli. Behavioral Neuroscience, 115(1), 33-42. https://doi.org/10.1037/0735-7044.115.1.33

Caravaggio, F., Plitman, E., Chung, J. K., Gerretsen, P., Kim, J., Iwata, Y., ... Graff-Guerrero, A. (2017). Trait impulsiveness is related to smaller post-commissural putamen volumes in males but not females. The European Journal of Neuroscience, 46(7), 2253-2264. https://doi.org/10.1111/ejn.13661

Carter, R. M., Macinnes, J. J., Huettel, S. A., \& Adcock, R. A. (2009). Activation in the VTA and nucleus accumbens increases in anticipation of both gains and losses. Frontiers in Behavioral Neuroscience, 3, 21. https://doi.org/10.3389/neuro.08.021.2009

Carver, C. S., \& White, T. L. (1994). Behavioral inhibition, behavioral activation, and affective responses to impending reward and punishment: The BIS/BAS Scales. Journal of Personality and Social Psychology, 67(2), 319-333. https://doi.org/10.1037/00223514.67.2.319 
Caseras, X., Àvila, C., \& Torrubia, R. (2003). The measurement of individual differences in Behavioural Inhibition and Behavioural Activation Systems: a comparison of personality scales. Personality and Individual Differences, 34(6), 999-1013. https://doi.org/10.1016/S0191-8869(02)00084-3

Cavanna, A. E. (2007). The precuneus and consciousness. CNS Spectrums, 12(7), 545-552. https://doi.org/10.1017/S1092852900021295

Cavanna, A. E., \& Trimble, M. R. (2006). The precuneus: A review of its functional anatomy and behavioural correlates. Brain, 129(3), 564-583. https://doi.org/10.1093/brain/awl004

Cherbuin, N., Windsor, T. D., Anstey, K. J., Maller, J. J., Meslin, C., \& Sachdev, P. S. (2008). Hippocampal volume is positively associated with behavioural inhibition (BIS) in a large community-based sample of mid-life adults: the PATH through life study. Social Cognitive and Affective Neuroscience, 3(3), 262-269. https://doi.org/10.1093/scan/nsn018

Choe, A. S., Jones, C. K., Joel, S. E., Muschelli, J., Belegu, V., Caffo, B. S., .. Pekar, J. J. (2015). Reproducibility and temporal structure in weekly resting-state fMRI over a period of 3.5 years. PLoS One, 10(10), e0140134. https://doi.org/10.1371/journal.pone.0140134

Clauss, J. A., Seay, A. L., VanDerKlok, R. M., Avery, S. N., Cao, A., Cowan, R. L., ... Blackford, J. U. (2014). Structural and functional bases of inhibited temperament. Social Cognitive and Affective Neuroscience, 9(12), 2049-2058. https://doi.org/10.1093/scan/nsu019

Clithero, J. A., \& Rangel, A. (2014). Informatic parcellation of the network involved in the computation of subjective value. Social Cognitive and Affective Neuroscience, 9(9), 12891302. https://doi.org/10.1093/scan/nst106

Cloninger, C. R. (1987). A Systematic Method for Clinical Description and Classification of Personality Variants: A Proposal. Archives of General Psychiatry, 44(6), 573-588. https://doi.org/10.1001/archpsyc.1987.01800180093014

Cloninger, C. R., Svrakic, D. M., \& Przybeck, T. R. (1993). A Psychobiological Model of Temperament and Character. Archives of General Psychiatry, 50(12), 975-990. https://doi.org/10.1001/archpsyc.1993.01820240059008

Cohen, M. X., Schoene-Bake, J.-C., Elger, C. E., \& Weber, B. (2009). Connectivity-based segregation of the human striatum predicts personality characteristics. Nature Neuroscience, 12(1), 32-34. https://doi.org/10.1038/nn.2228

Colder, C. R., Trucco, E. M., Lopez, H. I., Hawk, L. W., Read, J. P., Lengua, L. J., ... Eiden, R. D. (2011). Revised reinforcement sensitivity theory and laboratory assessment of BIS and BAS in children. Journal of Research in Personality, 45(2), 198-207. 
https://doi.org/10.1016/j.jrp.2011.01.005

Constantinou, E., Panayiotou, G., Konstantinou, N., Loutsiou-Ladd, A., \& Kapardis, A. (2011). Risky and aggressive driving in young adults: Personality matters. Accident; Analysis and Prevention, 43(4), 1323-1331. https://doi.org/10.1016/j.aap.2011.02.002

Cools, R. (2008). Role of dopamine in the motivational and cognitive control of behavior. Neuroscientist, 14(4), 381-395. https://doi.org/10.1177/1073858408317009

Cools, R., Calder, A. J., Lawrence, A. D., Clark, L., Bullmore, E., \& Robbins, T. W. (2005). Individual differences in threat sensitivity predict serotonergic modulation of amygdala response to fearful faces. Psychopharmacology, 180(4), 670-679. https://doi.org/10.1007/s00213-005-2215-5

Cools, R., Nakamura, K., \& Daw, N. D. (2011). Serotonin and dopamine: Unifying affective, activational, and decision functions. Neuropsychopharmacology, 36(1), 98-113. https://doi.org/10.1038/npp.2010.121

Corbetta, M., Patel, G., \& Shulman, G. L. (2008). The reorienting system of the human brain: from environment to theory of mind. Neuron, 58(3), 306-324. https://doi.org/10.1016/j.neuron.2008.04.017

Corr, P. J. (2002). J. A. Gray's reinforcement sensitivity theory: tests of the joint subsystems hypothesis of anxiety and impulsivity. Personality and Individual Differences, 33(4), 511532. https://doi.org/10.1016/S0191-8869(01)00170-2

Corr, P. J. (2004). Reinforcement sensitivity theory and personality. Neuroscience and Biobehavioral Reviews, 28(3), 317-332. https://doi.org/10.1016/j.neubiorev.2004.01.005

Corr, P. J. (2008). Reinforcement Sensitivity Theory (RST): introduction. In P. J. Corr (Ed.), The reinforcement sensitivity theory of personality (pp. 1-43). Cambridge: Cambridge University Press.

Corr, P. J. (2010). The psychoticism-psychopathy continuum: A neuropsychological model of core deficits. Personality and Individual Differences, 48(6), 695-703. https://doi.org/10.1016/j.paid.2009.12.023

Corr, P. J. (2016). Reinforcement Sensitivity Theory of Personality Questionnaires: Structural survey with recommendations. Personality and Individual Differences, 89, 60-64. https://doi.org/10.1016/j.paid.2015.09.045

Corr, P. J., \& Cooper, A. J. (2016). The Reinforcement Sensitivity Theory of Personality Questionnaire (RST-PQ): Development and Validation. Psychological Assessment, 28(11), 
1427-1440. https://doi.org/10.1037/pas0000273

Costa, P. T., \& McCrae, R. R. (1992). Revised NEO personality inventory (NEO-PI-R) and NEO five-factor inventory (NEO-FFI) manual. Odessa: Psychological Assessment Resources.

Costumero, V., Barrós-Loscertales, A., Bustamante, J. C., Ventura-Campos, N., Fuentes, P., \& Ávila, C. (2013). Reward sensitivity modulates connectivity among reward brain areas during processing of anticipatory reward cues. The European Journal of Neuroscience, 38(3), 2399-2407. https://doi.org/10.1111/ejn.12234

Costumero, V., Barrós-Loscertales, A., Bustamante, J. C., Ventura-Campos, N., Fuentes, P., Rosell-Negre, P., \& Ávila, C. (2013). Reward sensitivity is associated with brain activity during erotic stimulus processing. PLoS One, 8(6), e66940. https://doi.org/10.1371/journal.pone.0066940

Costumero, V., Barrós-Loscertales, A., Fuentes, P., Rosell-Negre, P., Bustamante, J. C., \& Ávila, C. (2016). BAS-drive trait modulates dorsomedial striatum activity during reward responseoutcome associations. Brain Imaging and Behavior, 10(3), 869-879. https://doi.org/10.1007/s11682-015-9466-5

Cremers, H. R., Demenescu, L. R., Aleman, A., Renken, R., van Tol, M. J., van der Wee, N. J. A., ... Roelofs, K. (2010). Neuroticism modulates amygdala-prefrontal connectivity in response to negative emotional facial expressions. NeuroImage, 49(1), 963-970. https://doi.org/10.1016/j.neuroimage.2009.08.023

Cunningham, W. A., Arbuckle, N. L., Jahn, A., Mowrer, S. M., \& Abduljalil, A. M. (2010). Aspects of neuroticism and the amygdala: Chronic tuning from motivational styles. Neuropsychologia, $\quad$ 48(12), 3399-3404. https://doi.org/10.1016/j.neuropsychologia.2010.06.026

D’Ardenne, K., McClure, S. M., Nystrom, L. E., \& Cohen, J. D. (2008). BOLD responses reflecting dopaminergic signals in the human ventral tegmental area. Science, 319(5867), 1264-1267. https://doi.org/10.1126/science.1150605

De Bellis, M. D., Casey, B. J., Dahl, R. E., Birmaher, B., Williamson, D. E., Thomas, K. M., ... Ryan, N. D. (2000). A pilot study of amygdala volumes in pediatric generalized anxiety disorder. Biological Psychiatry, $\quad 48(1), \quad 51-57$. https://doi.org/10.1016/s0006-3223(00)00835-0

Deakin, J. F., \& Graeff, F. G. (1991). 5-HT and mechanisms of defence. Journal of Psychopharmacology, 5(4), 305-315. https://doi.org/10.1177/026988119100500414

Delaparte, L., Bartlett, E., Grazioplene, R., Perlman, G., Gardus, J., DeLorenzo, C., ... Kotov, R. 
(2019). Structural correlates of the orbitofrontal cortex and amygdala and personality in female adolescents. Psychophysiology, 56(8), e13376. https://doi.org/10.1111/psyp.13376

Depue, R. A., \& Collins, P. F. (1999). Neurobiology of the structure of personality: dopamine, facilitation of incentive motivation, and extraversion. The Behavioral and Brain Sciences, 22(3), 491-517. https://doi.org/10.1017/s0140525x99002046

Derryberry, D., \& Reed, M. A. (1994). Temperament and attention: orienting toward and away from positive and negative signals. Journal of Personality and Social Psychology, 66(6), 1128-1139. https://doi.org/10.1037//0022-3514.66.6.1128

DeYoung, C. G. (2010). Personality Neuroscience and the Biology of Traits. Social and Personality Psychology Compass, 4(12), 1165-1180. https://doi.org/10.1111/j.17519004.2010.00327.x

Duncan, N. W., Wiebking, C., Tiret, B., Marjańska, M., Hayes, D. J., Lyttleton, O., ... Northoff, G. (2013). Glutamate Concentration in the Medial Prefrontal Cortex Predicts Resting-State Cortical-Subcortical Functional Connectivity in Humans. PLoS One, 8(4), e60312. https://doi.org/10.1371/journal.pone.0060312

Düzel, E., Bunzeck, N., Guitart-Masip, M., Wittmann, B., Schott, B. H., \& Tobler, P. N. (2009). Functional imaging of the human dopaminergic midbrain. Trends in Neurosciences, 32(6), 321-328. https://doi.org/10.1016/J.TINS.2009.02.005

Economides, M., Guitart-Masip, M., Kurth-Nelson, Z., \& Dolan, R. J. (2014). Anterior cingulate cortex instigates adaptive switches in choice by integrating immediate and delayed components of value in ventromedial prefrontal cortex. The Journal of Neuroscience, 34(9), 3340-3349. https://doi.org/10.1523/JNEUROSCI.4313-13.2014

Etkin, A., Büchel, C., \& Gross, J. J. (2015). The neural bases of emotion regulation. Nature Reviews. Neuroscience, 16(11), 693-700. https://doi.org/10.1038/nrn4044

Etkin, A., Egner, T., \& Kalisch, R. (2011). Emotional processing in anterior cingulate and medial prefrontal cortex. Trends in Cognitive Sciences, 15(2), 85-93. https://doi.org/10.1016/j.tics.2010.11.004

Etkin, A., Egner, T., Peraza, D. M., Kandel, E. R., \& Hirsch, J. (2006). Resolving Emotional Conflict: A Role for the Rostral Anterior Cingulate Cortex in Modulating Activity in the Amygdala. Neuron, 51(6), 871-882. https://doi.org/10.1016/j.neuron.2006.07.029

Etkin, A., Prater, K. E., Schatzberg, A. F., Menon, V., \& Greicius, M. D. (2009). Disrupted amygdalar subregion functional connectivity and evidence of a compensatory network in generalized anxiety disorder. Archives of General Psychiatry, 66(12), 1361-1372. 
https://doi.org/10.1001/archgenpsychiatry.2009.104

Ewbank, M. P., Lawrence, A. D., Passamonti, L., Keane, J., Peers, P. V., \& Calder, A. J. (2009). Anxiety predicts a differential neural response to attended and unattended facial signals of anger and fear. NeuroImage, 44(3), 1144-1151. https://doi.org/10.1016/j.neuroimage.2008.09.056

Eysenck, H. J. (1957). The dynamics of anxiety and hysteria. New York: Praeger.

Eysenck, H. J. (1960). Handbook of abnormal psychology. London: Pitman.

Eysenck, H. J. (1967). The biological basis of personality. Springfield: Thomas.

Eysenck, H. J. (1992). The definition and measurement of psychoticism. Personality and Individual Differences, 13(7), 757-785. https://doi.org/10.1016/0191-8869(92)90050-Y

Eysenck, H. J. (1997). Personality and experimental psychology: The unification of psychology and the possibility of a paradigm. Journal of Personality and Social Psychology, 73(6), 1224-1237. https://doi.org/10.1037/0022-3514.73.6.1224

Eysenck, H. J., \& Claridge, G. (1962). The position of hysterics and dysthymics in a twodimensional framework of personality description. Journal of Abnormal and Social Psychology, 64(1), 46-55. https://doi.org/10.1037/h0047528

Eysenck, H. J., \& Eysenck, M. W. (1985). Personality and individual differences: a natural science approach. New York: Plenum Press.

Eysenck, H. J., \& Eysenck, S. B. G. (1975). Manual of the Eysenck Personality Questionnaire (Junior and Adult). London: Hodder \& Stoughton.

Eysenck, H. J., \& Eysenck, S. B. G. (1976). Psychoticism as a dimension of personality. London: Hodder \& Stoughton.

Eysenck, S. B. G., Pearson, P. R., Easting, G., \& Allsopp, J. F. (1985). Age norms for impulsiveness, venturesomeness and empathy in adults. Personality and Individual Differences, 6(5), 613-619. https://doi.org/10.1016/0191-8869(85)90011-X

Fanselow, M. S. (1991). The Midbrain Periaqueductal Gray as a Coordinator of Action in Response to Fear and Anxiety. In A. Depaulis \& R. Bandler (Eds.), The Midbrain Periaqueductal Gray Matter (pp. 151-173). Boston: Springer. https://doi.org/10.1007/9781-4615-3302-3_10

Farrell, N., \& Walker, B. R. (2019). Reinforcement Sensitivity Theory and Problem Gambling in a General Population Sample. Journal of Gambling Studies, 35(4), 1163-1175. 
https://doi.org/10.1007/s10899-019-09850-3

Fatahi, Z., Haghparast, A., Khani, A., \& Kermani, M. (2018). Functional connectivity between anterior cingulate cortex and orbitofrontal cortex during value-based decision making. Neurobiology of Learning and Memory, 147, 74-78. https://doi.org/10.1016/J.NLM.2017.11.014

Fowles, D. C. (1980). The Three Arousal Model: Implications of Gray's Two-Factor Learning Theory for Heart Rate, Electrodermal Activity, and Psychopathy. Psychophysiology, 17(2), 87-104. https://doi.org/10.1111/j.1469-8986.1980.tb00117.x

Fowles, D. C. (2002). Biological Variables in Psychopathology: A Psychobiological Perspective. In P. B. Sutker \& H. E. Adams (Eds.), Comprehensive Handbook of Psychopathology (pp. 85-104). New York: Kluwer/Plenum. https://doi.org/10.1007/0-306-47377-1_4

Fox, E. (1994). Attentional Bias in Anxiety: A Defective Inhibition Hypothesis. Cognition and Emotion, 8(2), 165-195. https://doi.org/10.1080/02699939408408934

Fox, E. (1996). Selective processing of threatening words in anxiety: The role of awareness. Cognition and Emotion, 10(5), 449-480. https://doi.org/10.1080/026999396380114

Franken, I. H. A., \& Muris, P. (2006). BIS/BAS personality characteristics and college students' substance use. Personality and Individual Differences, 40(7), 1497-1503. https://doi.org/10.1016/j.paid.2005.12.005

Franken, I. H. A., Muris, P., \& Georgieva, I. (2006). Gray’s model of personality and addiction. Addictive Behaviors, 31(3), 399-403. https://doi.org/10.1016/j.addbeh.2005.05.022

Frühholz, S., Prinz, M., \& Herrmann, M. (2010). Affect-related personality traits and contextual interference processing during perception of facial affect. Neuroscience Letters, 469(2), 260-264. https://doi.org/10.1016/j.neulet.2009.12.010

Fuentes-Claramonte, P., Ávila, C., Rodríguez-Pujadas, A., Costumero, V., Ventura-Campos, N., Bustamante, J. C., ... Barrós-Loscertales, A. (2016a). Characterizing individual differences in reward sensitivity from the brain networks involved in response inhibition. NeuroImage, 124(Pt A), 287-299. https://doi.org/10.1016/j.neuroimage.2015.08.067

Fuentes-Claramonte, P., Ávila, C., Rodríguez-Pujadas, A., Costumero, V., Ventura-Campos, N., Bustamante, J. C., ... Barrós-Loscertales, A. (2016b). Inferior frontal cortex activity is modulated by reward sensitivity and performance variability. Biological Psychology, 114, 127-137. https://doi.org/10.1016/j.biopsycho.2016.01.001

Fuentes-Claramonte, P., Ávila, C., Rodríguez-Pujadas, A., Ventura-Campos, N., Bustamante, J. 
C., Costumero, V., ... Barrós-Loscertales, A. (2015). Reward Sensitivity Modulates Brain Activity in the Prefrontal Cortex, ACC and Striatum during Task Switching. PLoS One, 10(4), e0123073. https://doi.org/10.1371/journal.pone.0123073

Fuentes, P., Barrós-Loscertales, A., Bustamante, J. C., Rosell, P., Costumero, V., \& Ávila, C. (2012). Individual differences in the Behavioral Inhibition System are associated with orbitofrontal cortex and precuneus gray matter volume. Cognitive, Affective \& Behavioral Neuroscience, 12(3), 491-498. https://doi.org/10.3758/s13415-012-0099-5

Fuentes, P., Barrós-Loscertales, A., Rodríguez-Pujadas, A., Ventura-Campos, N., Bustamante, J. C., Costumero, V., ... Ávila, C. (2014a). Behavioral Activation System modulation of brain activity during task switching. Personality and Individual Differences, 60, S52. https://doi.org/10.1016/J.PAID.2013.07.212

Fuentes, P., Barrós-Loscertales, A., Rodríguez-Pujadas, A., Ventura-Campos, N., Bustamante, J. C., Costumero, V., ... Ávila, C. (2014b). Reward sensitivity modulation of brain activity during response inhibition. Personality and Individual Differences, 60, S69. https://doi.org/10.1016/J.PAID.2013.07.303

Gardini, S., Cloninger, C. R., \& Venneri, A. (2009). Individual differences in personality traits reflect structural variance in specific brain regions. Brain Research Bulletin, 79(5), 265270. https://doi.org/10.1016/j.brainresbull.2009.03.005

Gentili, C., Cristea, I. A., Ricciardi, E., Vanello, N., Popita, C., David, D., \& Pietrini, P. (2017). Not in one metric: Neuroticism modulates different resting state metrics within distinctive brain regions. Behavioural Brain Research, 327, 34-43. https://doi.org/10.1016/j.bbr.2017.03.031

Glashouwer, K. A., Bloot, L., Veenstra, E. M., Franken, I. H. A., \& de Jong, P. J. (2014). Heightened sensitivity to punishment and reward in anorexia nervosa. Appetite, 75, 97-102. https://doi.org/10.1016/j.appet.2013.12.019

Gray, J. A. (1970). The psychophysiological basis of introversion-extraversion. Behaviour Research and Therapy, 8(3), 249-266. https://doi.org/10.1016/0005-7967(70)90069-0

Gray, J. A. (1981). A Critique of Eysenck's Theory of Personality. In H. J. Eysenck (Ed.), A Model for Personality (pp. 246-276). Berlin: Springer. https://doi.org/10.1007/978-3-64267783-0_8

Gray, J. A. (1982). The neuropsychology of anxiety: An enquiry into the functions of the septohippocampal system. Oxford: Oxford University Press.

Gray, J. A. (1987a). Perspectives on anxiety and impulsivity: A commentary. Journal of Research 
in Personality, 21(4), 493-509. https://doi.org/10.1016/0092-6566(87)90036-5

Gray, J. A. (1987b). The neuropsychology of emotion and personality. In S. M. Stahl, S. D. Iversen, \& E. C. Goodman (Eds.), Cognitive neurochemistry (pp. 171-190). Oxford: Oxford University Press.

Gray, J. A. (1987c). The psychology of fear and stress. Cambridge: Cambridge University Press.

Gray, J. A. (1995). A model of the limbic system and basal ganglia: applications to anxiety and schizophrenia. In M. S. Gazzaniga (Ed.), The cognitive neurosciences (pp. 1165-1176). Cambridge: MIT Press.

Gray, J. A., \& McNaughton, N. (2000). The neuropsychology of anxiety: An enquiry into the functions of the septo-hippocampal system (2nd ed.). Oxford: Oxford University Press.

Gross, C. T., \& Canteras, N. S. (2012). The many paths to fear. Nature Reviews. Neuroscience, 13(9), 651-658. https://doi.org/10.1038/nrn3301

Haber, S. N. (2014). The place of dopamine in the cortico-basal ganglia circuit. Neuroscience, 282, 248-257. https://doi.org/10.1016/J.NEUROSCIENCE.2014.10.008

Haber, S. N., \& Knutson, B. (2010). The reward circuit: linking primate anatomy and human imaging. Neuropsychopharmacology, 35(1), 4-26. https://doi.org/10.1038/npp.2009.129

Hahn, T., Dresler, T., Ehlis, A.-C., Plichta, M. M., Heinzel, S., Polak, T., ... Fallgatter, A. J. (2009). Neural response to reward anticipation is modulated by Gray's impulsivity. NeuroImage, 46(4), 1148-1153. https://doi.org/10.1016/j.neuroimage.2009.03.038

Hahn, T., Dresler, T., Plichta, M. M., Ehlis, A.-C., Ernst, L. H., Markulin, F., .. Fallgatter, A. J. (2010). Functional amygdala-hippocampus connectivity during anticipation of aversive events is associated with Gray's trait "sensitivity to punishment”. Biological Psychiatry, 68(5), 459-464. https://doi.org/10.1016/j.biopsych.2010.04.033

Hahn, T., Heinzel, S., Notebaert, K., Dresler, T., Reif, A., Lesch, K.-P., ... Fallgatter, A. J. (2013). The tricks of the trait: Neural implementation of personality varies with genotype-dependent $\begin{array}{llll}\text { serotonin } & \text { levels. } & \text { NeuroImage, } & 81,\end{array}$ https://doi.org/10.1016/J.NEUROIMAGE.2013.05.037

Harmelech, T., \& Malach, R. (2013). Neurocognitive biases and the patterns of spontaneous correlations in the human cortex. Trends in Cognitive Sciences, 17(12), 606-615. https://doi.org/10.1016/j.tics.2013.09.014

Hayes, D. J., Duncan, N. W., Xu, J., \& Northoff, G. (2014). A comparison of neural responses to appetitive and aversive stimuli in humans and other mammals. Neuroscience and 
Biobehavioral Reviews, 45, 350-368. https://doi.org/10.1016/j.neubiorev.2014.06.018

Hernández, M., Palomar-García, M. Á., Nohales-Nieto, B., Olcina-Sempere, G., VillarRodríguez, E., Pastor, R., ... Parcet, M. A. (2019). Separate Contribution of Striatum Volume and Pitch Discrimination to Individual Differences in Music Reward. Psychological Science, 30(9), 1352-1361. https://doi.org/10.1177/0956797619859339

Hiser, J., \& Koenigs, M. (2018). The Multifaceted Role of the Ventromedial Prefrontal Cortex in Emotion, Decision Making, Social Cognition, and Psychopathology. Biological Psychiatry, 83(8), 638-647. https://doi.org/10.1016/J.BIOPSYCH.2017.10.030

Hogan, P. S., Galaro, J. K., \& Chib, V. S. (2019). Roles of Ventromedial Prefrontal Cortex and Anterior Cingulate in Subjective Valuation of Prospective Effort. Cerebral Cortex, 29(10), 4277-4290. https://doi.org/10.1093/cercor/bhy310

Holmes, A. J., Hollinshead, M. O., Roffman, J. L., Smoller, J. W., \& Buckner, R. L. (2016). Individual Differences in Cognitive Control Circuit Anatomy Link Sensation Seeking, Impulsivity, and Substance Use. The Journal of Neuroscience, 36(14), 4038-4049. https://doi.org/10.1523/JNEUROSCI.3206-15.2016

Holmes, A. J., Lee, P. H., Hollinshead, M. O., Bakst, L., Roffman, J. L., Smoller, J. W., \& Buckner, R. L. (2012). Individual differences in amygdala-medial prefrontal anatomy link negative affect, impaired social functioning, and polygenic depression risk. The Journal of Neuroscience, 32(50), 18087-18100. https://doi.org/10.1523/JNEUROSCI.2531-12.2012

Huckins, J. F., Adeyemo, B., Miezin, F. M., Power, J. D., Gordon, E. M., Laumann, T. O., ... Kelley, W. M. (2018). Reward-related regions form a preferentially coupled system at rest. Human Brain Mapping, 40(2), 361-376. https://doi.org/10.1002/hbm.24377

Hundt, N. E., Kimbrel, N. A., Mitchell, J. T., \& Nelson-Gray, R. O. (2008). High BAS, but not low BIS, predicts externalizing symptoms in adults. Personality and Individual Differences, 44(3), 565-575. https://doi.org/10.1016/j.paid.2007.09.018

Hundt, N. E., Nelson-Gray, R. O., Kimbrel, N. A., Mitchell, J. T., \& Kwapil, T. R. (2007). The interaction of reinforcement sensitivity and life events in the prediction of anhedonic depression and mixed anxiety-depression symptoms. Personality and Individual Differences, 43(5), 1001-1012. https://doi.org/10.1016/j.paid.2007.02.021

Iidaka, T., Matsumoto, A., Ozaki, N., Suzuki, T., Iwata, N., Yamamoto, Y., ... Sadato, N. (2006). Volume of left amygdala subregion predicted temperamental trait of harm avoidance in female young subjects. A voxel-based morphometry study. Brain Research, 1125(1), 8593. https://doi.org/10.1016/j.brainres.2006.09.015 
Ikemoto, S. (2007). Dopamine reward circuitry: Two projection systems from the ventral midbrain to the nucleus accumbens-olfactory tubercle complex. Brain Research Reviews, 56(1), 27-78. https://doi.org/10.1016/j.brainresrev.2007.05.004

Jackson, C. J. (2009). Jackson-5 scales of revised Reinforcement Sensitivity Theory (r-RST) and their application to dysfunctional real world outcomes. Journal of Research in Personality, 43(4), 556-569. https://doi.org/10.1016/j.jrp.2009.02.007

Jackson, C. J., \& Smillie, L. D. (2004). Appetitive motivation predicts the majority of personality and an ability measure: A comparison of BAS measures and a re-evaluation of the importance of RST. Personality and Individual Differences, 36(7), 1627-1636. https://doi.org/10.1016/j.paid.2003.06.010

Jao, T., Vértes, P. E., Alexander-Bloch, A. F., Tang, I.-N., Yu, Y.-C., Chen, J.-H., \& Bullmore, E. T. (2013). Volitional eyes opening perturbs brain dynamics and functional connectivity regardless of light input. NeuroImage, 69, 21-34. https://doi.org/10.1016/j.neuroimage.2012.12.007

Jean-Richard-Dit-Bressel, P., Killcross, S., \& McNally, G. P. (2018). Behavioral and neurobiological mechanisms of punishment: Implications for psychiatric disorders. Neuropsychopharmacology, 43(8), 1639-1650. https://doi.org/10.1038/s41386-018-0047-3

Kahnt, T., Chang, L. J., Park, S. Q., Heinzle, J., \& Haynes, J. D. (2012). Connectivity-based parcellation of the human orbitofrontal cortex. The Journal of Neuroscience, 32(18), 62406250. https://doi.org/10.1523/JNEUROSCI.0257-12.2012

Kane, T. A., Loxton, N. J., Staiger, P. K., \& Dawe, S. (2004). Does the tendency to act impulsively underlie binge eating and alcohol use problems? An empirical investigation. Personality and Individual Differences, 36(1), 83-94. https://doi.org/10.1016/S0191-8869(03)00070-9

Kasch, K. L., Rottenberg, J., Arnow, B. A., \& Gotlib, I. H. (2002). Behavioral activation and inhibition systems and the severity and course of depression. Journal of Abnormal Psychology, 111(4), 589-597. https://doi.org/10.1037//0021-843x.111.4.589

Kennis, M., Rademaker, A. R., \& Geuze, E. (2013). Neural correlates of personality: an integrative review. Neuroscience and Biobehavioral Reviews, 37(1), 73-95. https://doi.org/10.1016/j.neubiorev.2012.10.012

Kim, M. J., Gee, D. G., Loucks, R. A., Davis, F. C., \& Whalen, P. J. (2011). Anxiety dissociates dorsal and ventral medial prefrontal cortex functional connectivity with the amygdala at rest. Cerebral Cortex, 21(7), 1667-1673. https://doi.org/10.1093/cercor/bhq237

Kimbrel, N. A. (2008). A model of the development and maintenance of generalized social 
$\begin{array}{llll}\text { phobia. } \quad \text { Clinical } & \text { Psychology }\end{array}$ https://doi.org/10.1016/j.cpr.2007.08.003

Kimbrel, N. A., Nelson-Gray, R. O., \& Mitchell, J. T. (2007). Reinforcement sensitivity and maternal style as predictors of psychopathology. Personality and Individual Differences, 42(6), 1139-1149. https://doi.org/10.1016/j.paid.2006.06.028

Knyazev, G. G. (2004). Behavioural activation as predictor of substance use: mediating and moderating role of attitudes and social relationships. Drug and Alcohol Dependence, 75(3), 309-321. https://doi.org/10.1016/j.drugalcdep.2004.03.007

Koelsch, S., Skouras, S., \& Jentschke, S. (2013). Neural Correlates of Emotional Personality: A Structural and Functional Magnetic Resonance Imaging Study. PLoS One, 8(11), e77196. https://doi.org/10.1371/journal.pone.0077196

Korponay, C., Dentico, D., Kral, T., Ly, M., Kruis, A., Goldman, R., ... Davidson, R. J. (2017). Neurobiological correlates of impulsivity in healthy adults: Lower prefrontal gray matter volume and spontaneous eye-blink rate but greater resting-state functional connectivity in basal ganglia-thalamo-cortical circuitry. NeuroImage, 157, 288-296. https://doi.org/10.1016/j.neuroimage.2017.06.015

Krebs, R. M., Heipertz, D., Schuetze, H., \& Duzel, E. (2011). Novelty increases the mesolimbic functional connectivity of the substantia nigra/ventral tegmental area (SN/VTA) during reward anticipation: Evidence from high-resolution fMRI. NeuroImage, 58(2), 647-655. https://doi.org/10.1016/j.neuroimage.2011.06.038

Krebs, R. M., Schott, B. H., \& Düzel, E. (2009). Personality traits are differentially associated with patterns of reward and novelty processing in the human substantia nigra/ventral tegmental area. Biological Psychiatry, 65(2), 103-110. https://doi.org/10.1016/j.biopsych.2008.08.019

Krug, M. K., \& Carter, C. S. (2010). Adding fear to conflict: A general purpose cognitive control network is modulated by trait anxiety. Cognitive, Affective \& Behavioral Neuroscience, 10(3), 357-371. https://doi.org/10.3758/CABN.10.3.357

Kyeong, S., Kim, E., Park, H. J., \& Hwang, D. U. (2014). Functional network organizations of two contrasting temperament groups in dimensions of novelty seeking and harm avoidance. Brain Research, 1575, 33-44. https://doi.org/10.1016/j.brainres.2014.05.037

Lawson, A. L., Liu, X., Joseph, J., Vagnini, V. L., Kelly, T. H., \& Jiang, Y. (2012). Sensation seeking predicts brain responses in the old-new task: Converging multimodal neuroimaging evidence. International Journal of Psychophysiology, 84(3), 260-269. 
https://doi.org/10.1016/j.ijpsycho.2012.03.003

LeDoux, J. E. (1994). Emotion, memory and the brain. Scientific American, 270(6), 50-57. https://doi.org/ 10.1038/scientificamerican0694-50

LeDoux, J. E. (2000). Emotion circuits in the brain. Annual Review of Neuroscience, 23, 155184. https://doi.org/10.1146/annurev.neuro.23.1.155

LeDoux, J. E. (2014). Coming to terms with fear. Proceedings of the National Academy of Sciences of the United States of America, 111(8), 2871-2878. https://doi.org/10.1073/pnas.1400335111

LeDoux, J. E., Iwata, J., Cicchetti, P., \& Reis, D. J. (1988). Different projections of the central amygdaloid nucleus mediate autonomic and behavioral correlates of conditioned fear. The $\begin{array}{llll}\text { Journal of } & \text { Neuroscience, }\end{array}$ https://doi.org/10.1523/JNEUROSCI.08-07-02517.1988

Lei, X., Chen, C., Xue, F., He, Q., Chen, C., Liu, Q., ... Dong, Q. (2014). Fiber connectivity between the striatum and cortical and subcortical regions is associated with temperaments in $\quad$ Chinese males. $\quad$ NeuroImage, $89, \quad 226-234$. https://doi.org/10.1016/j.neuroimage.2013.04.043

Lerner, D. A., Hatak, I., \& Rauch, A. (2018). Deep roots? Behavioral Inhibition and Behavioral Activation System (BIS/BAS) sensitivity and entrepreneurship. Journal of Business Venturing Insights, 9, 107-115. https://doi.org/10.1016/J.JBVI.2018.02.005

Levita, L., Bois, C., Healey, A., Smyllie, E., Papakonstantinou, E., Hartley, T., \& Lever, C. (2014). The Behavioural Inhibition System, anxiety and hippocampal volume in a nonclinical population. Biology of Mood \& Anxiety Disorders, 4(1), 4. https://doi.org/10.1186/2045-5380-4-4

Li, S., Demenescu, L. R., Sweeney-Reed, C. M., Krause, A. L., Metzger, C. D., \& Walter, M. (2017). Novelty seeking and reward dependence-related large-scale brain networks functional connectivity variation during salience expectancy. Human Brain Mapping, 38(8), 4064-4077. https://doi.org/10.1002/hbm.23648

Li, W., Cui, H., Zhu, Z., Kong, L., Guo, Q., Zhu, Y., ... Li, C. (2016). Aberrant Functional Connectivity between the Amygdala and the Temporal Pole in Drug-Free Generalized Anxiety Disorder. Frontiers in Human Neuroscience, 10, 549. https://doi.org/10.3389/fnhum.2016.00549

Li, Yadan, Qiao, L., Sun, J., Wei, D., Li, W., Qiu, J., ... Shi, H. (2014). Gender-specific neuroanatomical basis of behavioral inhibition/approach systems (BIS/BAS) in a large 
sample of young adults: a voxel-based morphometric investigation. Behavioural Brain Research, 274, 400-408. https://doi.org/10.1016/j.bbr.2014.08.041

Li, Ying, Qin, W., Jiang, T., Zhang, Y., \& Yu, C. (2012). Sex-dependent correlations between the personality dimension of harm avoidance and the resting-state functional connectivity of $\begin{array}{lllll}\text { amygdala } & \text { subregions. } & P L o S & \text { One, } & 7(4),\end{array}$ https://doi.org/10.1371/journal.pone.0035925

Linnman, C., Moulton, E. A., Barmettler, G., Becerra, L., \& Borsook, D. (2012). Neuroimaging of the periaqueductal gray: State of the field. NeuroImage, 60(1), 505-522. https://doi.org/10.1016/j.neuroimage.2011.11.095

Liu, D., Dong, Z., Zuo, X., Wang, J., \& Zang, Y. (2013). Eyes-open/eyes-closed dataset sharing for reproducibility evaluation of resting state fMRI data analysis methods. Neuroinformatics, 11(4), 469-476. https://doi.org/10.1007/s12021-013-9187-0

Locke, H. S., \& Braver, T. S. (2008). Motivational influences on cognitive control: behavior, brain activation, and individual differences. Cognitive, Affective \& Behavioral Neuroscience, 8(1), 99-112. https://doi.org/10.3758/cabn.8.1.99

Lowry, C. A., Johnson, P. L., Hay-Schmidt, A., Mikkelsen, J., \& Shekhar, A. (2005). Modulation of anxiety circuits by serotonergic systems. Stress, 8(4), 233-246. https://doi.org/10.1080/10253890500492787

Loxton, N. J., \& Dawe, S. (2006). Reward and punishment sensitivity in dysfunctional eating and hazardous drinking women: Associations with family risk. Appetite, 47(3), 361-371. https://doi.org/10.1016/j.appet.2006.05.014

Maack, D. J., Buchanan, E., \& Young, J. (2015). Development and Psychometric Investigation of an Inventory to Assess Fight, Flight, and Freeze Tendencies: The Fight, Flight, Freeze Questionnaire. Cognitive Behaviour Therapy, 44(2), 117-127. https://doi.org/10.1080/16506073.2014.972443

Maack, D. J., Tull, M. T., \& Gratz, K. L. (2012). Examining the incremental contribution of behavioral inhibition to generalized anxiety disorder relative to other Axis I disorders and cognitive-emotional vulnerabilities. Journal of Anxiety Disorders, 26(6), 689-695. https://doi.org/10.1016/j.janxdis.2012.05.005

MacAndrew, C., \& Steele, T. (1991). Gray's behavioral inhibition system: A psychometric examination. Personality and Individual Differences, 12(2), 157-171. https://doi.org/10.1016/0191-8869(91)90099-W

Madsen, K. S., Jernigan, T. L., Iversen, P., Frokjaer, V. G., Mortensen, E. L., Knudsen, G. M., \& 
Baaré, W. F. C. (2012). Cortisol awakening response and negative emotionality linked to asymmetry in major limbic fibre bundle architecture. Psychiatry Research: Neuroimaging, 201(1), 63-72. https://doi.org/10.1016/j.pscychresns.2011.07.015

Mathews, A., May, J., Mogg, K., \& Eysenck, M. (1990). Attentional bias in anxiety: Selective search or defective filtering? Journal of Abnormal Psychology, 99(2), 166-173. https://doi.org/10.1037/0021-843X.99.2.166

Mathews, A., Yiend, J., \& Lawrence, A. D. (2004). Individual differences in the modulation of fear-related brain activation by attentional control. Journal of Cognitive Neuroscience, 16(10), 1683-1694. https://doi.org/10.1162/0898929042947810

Matsuo, K., Nicoletti, M., Nemoto, K., Hatch, J. P., Peluso, M. A. M., Nery, F. G., \& Soares, J. C. (2009). A voxel-based morphometry study of frontal gray matter correlates of impulsivity. Human Brain Mapping, 30(4), 1188-1195. https://doi.org/10.1002/hbm.20588

Matthews, G., \& Gilliland, K. (1999). The personality theories of H. J. Eysenck and J. A. Gray: A comparative review. Personality and Individual Differences, 26(4), 583-626. https://doi.org/10.1016/S0191-8869(98)00158-5

Matton, A., Goossens, L., Braet, C., \& Vervaet, M. (2013). Punishment and Reward Sensitivity: Are Naturally Occurring Clusters in These Traits Related to Eating and Weight Problems in Adolescents? European Eating Disorders Review, 21(3), 184-194. https://doi.org/10.1002/erv.2226

Matton, A., Goossens, L., Vervaet, M., \& Braet, C. (2015). Temperamental differences between adolescents and young adults with or without an eating disorder. Comprehensive Psychiatry, 56, 229-238. https://doi.org/10.1016/J.COMPPSYCH.2014.09.005

McClure, S. M., York, M. K., \& Montague, P. R. (2004). The neural substrates of reward processing in humans: the modern role of FMRI. The Neuroscientist, 10(3), 260-268. https://doi.org/10.1177/1073858404263526

McNaughton, N., \& Corr, P. J. (2004). A two-dimensional neuropsychology of defense: fear/anxiety and defensive distance. Neuroscience and Biobehavioral Reviews, 28(3), 285305. https://doi.org/10.1016/j.neubiorev.2004.03.005

McNaughton, N., \& Corr, P. J. (2008). The neuropsychology of fear and anxiety: a foundation for Reinforcement Sensitivity Theory. In P. J. Corr (Ed.), The reinforcement sensitivity theory of personality (pp. 44-94). Cambridge: Cambridge University Press.

McNaughton, N., \& Corr, P. J. (2018). Survival circuits and risk assessment. Current Opinion in Behavioral Sciences, 24, 14-20. https://doi.org/10.1016/j.cobeha.2018.01.018 
McNaughton, N., \& Corr, P. J. (2019). Hierarchical Levels of Control: The State-Trait Distinction. $\quad$ Psychological Inquiry, 30(3), 158-164. https://doi.org/10.1080/1047840X.2019.1646058

McNaughton, N., DeYoung, C. G., \& Corr, P. J. (2016). Approach/Avoidance. In J. R. Absher \& J. Cloutier (Eds.), Neuroimaging Personality, Social Cognition, and Character (pp. 25-49). San Diego: Elsevier. https://doi.org/10.1016/B978-0-12-800935-2.00002-6

Mellet, E., Petit, L., Mazoyer, B., Denis, M., \& Tzourio, N. (1998). Reopening the mental imagery debate: lessons from functional anatomy. NeuroImage, 8(2), 129-139. https://doi.org/10.1006/nimg.1998.0355

Merz, E. C., He, X., \& Noble, K. G. (2018). Anxiety, depression, impulsivity, and brain structure in children and adolescents. NeuroImage: Clinical, 20, 243-251. https://doi.org/10.1016/j.nicl.2018.07.020

Mincic, A. M. (2015). Neuroanatomical correlates of negative emotionality-related traits: A systematic review and meta-analysis. Neuropsychologia, 77, 97-118. https://doi.org/10.1016/j.neuropsychologia.2015.08.007

Mitchell, J. T., \& Nelson-Gray, R. O. (2006). Attention-Deficit/Hyperactivity Disorder symptoms in adults: Relationship to Gray's Behavioral Approach System. Personality and Individual Differences, 40(4), 749-760. https://doi.org/10.1016/j.paid.2005.08.011

Mobbs, D., \& Kim, J. J. (2015). Neuroethological studies of fear, anxiety, and risky decisionmaking in rodents and humans. Current Opinion in Behavioral Sciences, 5, 8-15. https://doi.org/10.1016/j.cobeha.2015.06.005

Mogg, K., \& Bradley, B. P. (2002). Selective orienting of attention to masked threat faces in social anxiety. Behaviour Research and Therapy, 40(12), 1403-1414. https://doi.org/10.1016/S0005-7967(02)00017-7

Mogg, K., \& Bradley, B. P. (2018). Anxiety and Threat-Related Attention: CognitiveMotivational Framework and Treatment. Trends in Cognitive Sciences, 22(3), 225-240. https://doi.org/10.1016/j.tics.2018.01.001

Mogg, K., Bradley, B. P., Williams, R., \& Mathews, A. (1993). Subliminal processing of emotional information in anxiety and depression. Journal of Abnormal Psychology, 102(2), 304-311. https://doi.org/10.1037/0021-843X.102.2.304

Mogg, K., Garner, M., \& Bradley, B. P. (2007). Anxiety and orienting of gaze to angry and fearful $\begin{array}{lll}\text { faces. } & \text { Biological } & \text { Psychology, }\end{array}$ https://doi.org/10.1016/j.biopsycho.2007.07.005 
Mohammadi, B., Hammer, A., Miedl, S. F., Wiswede, D., Marco-Pallarés, J., Herrmann, M., ... Münte, F. (2016). Intertemporal choice behavior is constrained by brain structure in healthy participants and pathological gamblers. Brain Structure and Function, 221(6), 3157-3170. https://doi.org/10.1007/s00429-015-1093-9

Montag, C., Markowetz, A., Blaszkiewicz, K., Andone, I., Lachmann, B., Sariyska, R., ... Markett, S. (2017). Facebook usage on smartphones and gray matter volume of the nucleus accumbens. Behavioural Brain Research, 329, 221-228. https://doi.org/10.1016/J.BBR.2017.04.035

Montag, C., Reuter, M., Weber, B., Markett, S., \& Schoene-Bake, J. C. (2012). Individual differences in trait anxiety are associated with white matter tract integrity in the left temporal lobe in healthy males but not females. Neuroscience, 217, 77-83. https://doi.org/10.1016/j.neuroscience.2012.05.017

Montague, P. R., Hyman, S. E., \& Cohen, J. D. (2004). Computational roles for dopamine in behavioural control. Nature, 431(7010), 760-767. https://doi.org/10.1038/nature03015

Mortensen, J. A., Lehn, H., Evensmoen, H. R., \& Håberg, A. K. (2015). Evidence for an antagonistic interaction between reward and punishment sensitivity on striatal activity: A verification of the Joint Subsystems Hypothesis. Personality and Individual Differences, 74, 214-219. https://doi.org/10.1016/J.PAID.2014.10.023

Na, E., Lee, H., Choi, I., \& Kim, D.-J. (2017). Comorbidity of Internet gaming disorder and alcohol use disorder: A focus on clinical characteristics and gaming patterns. The American Journal on Addictions, 26(4), 326-334. https://doi.org/10.1111/ajad.12528

Newman, J. P., MacCoon, D. G., Vaughn, L. J., \& Sadeh, N. (2005). Validating a distinction between primary and secondary psychopathy with measures of Gray's BIS and BAS constructs. Journal of Abnormal Psychology, 114(2), 319-323. https://doi.org/10.1037/0021-843X.114.2.319

Newman, J. P., \& Wallace, J. F. (1993). Diverse pathways to deficient self-regulation: Implications for disinhibitory psychopathology in children. Clinical Psychology Review, 13(8), 699-720. https://doi.org/10.1016/S0272-7358(05)80002-9

Newman, J. P., Widom, C. S., \& Nathan, S. (1985). Passive avoidance in syndromes of disinhibition: psychopathy and extraversion. Journal of Personality and Social Psychology, 48(5), 1316-1327. https://doi.org/10.1037//0022-3514.48.5.1316

Nigg, J. T. (2001). Is ADHD a disinhibitory disorder? Psychological Bulletin, 127(5), 571-598. https://doi.org/10.1037/0033-2909.127.5.571 
Nostro, A. D., Müller, V. I., Reid, A. T., \& Eickhoff, S. B. (2016). Correlations Between Personality and Brain Structure: A Crucial Role of Gender. Cerebral Cortex, 27(7), 36983712. https://doi.org/10.1093/cercor/bhw191

Nostro, A. D., Müller, V. I., Varikuti, D. P., Pläschke, R. N., Hoffstaedter, F., Langner, R., ... Eickhoff, S. B. (2018). Predicting personality from network-based resting-state functional connectivity. Brain Structure and Function, 223(6), 2699-2719. https://doi.org/10.1007/s00429-018-1651-z

O’Connor, R. M., Stewart, S. H., \& Watt, M. C. (2009). Distinguishing BAS risk for university students' drinking, smoking, and gambling behaviors. Personality and Individual Differences, 46(4), 514-519. https://doi.org/10.1016/j.paid.2008.12.002

Ormel, J., Bastiaansen, A., Riese, H. H., Bos, E. H., Servaas, M., Ellenbogen, M., ... Aleman, A. A. (2013). The biological and psychological basis of neuroticism: Current status and future directions. Neuroscience and Biobehavioral Reviews, 37(1), 59-72. https://doi.org/10.1016/j.neubiorev.2012.09.004

Pang, Y., Cui, Q., Wang, Y., Chen, Y., Wang, X., Han, S., ... Chen, H. (2016). Extraversion and neuroticism related to the resting-state effective connectivity of amygdala. Scientific Reports, 6(1), 35484. https://doi.org/10.1038/srep35484

Panizzon, M. S., Fennema-Notestine, C., Eyler, L. T., Jernigan, T. L., Prom-Wormley, E., Neale, M., ... Kremen, W. S. (2009). Distinct Genetic Influences on Cortical Surface Area and $\begin{array}{llll}\text { Cortical Thickness. Cerebral } & \text { Cortex, }\end{array}$ https://doi.org/10.1093/cercor/bhp026

Pardo, Y., Aguilar, R., Molinuevo, B., \& Torrubia, R. (2007). Alcohol use as a behavioural sign of disinhibition: evidence from J.A. Gray's model of personality. Addictive Behaviors, 32(10), 2398-2403. https://doi.org/10.1016/j.addbeh.2007.02.010

Pastor, M. C., Ross, S. R., Segarra, P., Montañés, S., Poy, R., \& Moltó, J. (2007). Behavioral inhibition and activation dimensions: Relationship to MMPI-2 indices of personality disorder. Personality and Individual Differences, 42(2), 235-245. https://doi.org/10.1016/J.PAID.2006.06.015

Perkins, A. M., Kemp, S. E., \& Corr, P. J. (2007). Fear and anxiety as separable emotions: An investigation of the revised reinforcement sensitivity theory of personality. Emotion, 7(2), 252-261. https://doi.org/10.1037/1528-3542.7.2.252

Petersen, S. E., \& Posner, M. I. (2012). The Attention System of the Human Brain: 20 Years After. Annual Review of Neuroscience, 35(1), 73-89. https://doi.org/10.1146/annurev- 
neuro-062111-150525

Picerni, E., Petrosini, L., Piras, F., Laricchiuta, D., Cutuli, D., Chiapponi, C., ... Spalletta, G. (2013). New evidence for the cerebellar involvement in personality traits. Frontiers in Behavioral Neuroscience, 7, 133. https://doi.org/10.3389/fnbeh.2013.00133

Pickering, A. D., \& Corr, P. J. (2008). J.A. Gray's reinforcement sensitivity theory (RST) of personality. In G. J. Boyle, G. Matthews, \& D. H. Saklofske (Eds.), The SAGE handbook of personality theory and assessment, Vol 1: Personality theories and models (pp. 239-256). London: SAGE Publications. https://doi.org/10.4135/9781849200462.n11

Pickering, A. D., Corr, P. J., \& Gray, J. A. (1999). Interactions and reinforcement sensitivity theory: A theoretical analysis of Rusting and Larsen (1997). Personality and Individual Differences, 26(2), 357-365. https://doi.org/10.1016/S0191-8869(98)00019-1

Pickering, A. D., \& Gray, J. A. (1999). The neuroscience of personality. In L. A. Pervin \& O. P. John (Eds.), Handbook of personality: Theory and research (2nd edition) (pp. 277-299). New York: Guilford Press.

Pickering, A. D., \& Gray, J. A. (2001). Dopamine, appetitive reinforcement, and the neuropsychology of human learning: An individual differences approach. In A. Elias \& A. Angleitner (Eds.), Advances in individual differences research (pp. 113-149). Lengerich: PABST Science Publishers.

Pickering, A. D., \& Smillie, L. D. (2008). The behavioural activation system: Challenges and opportunities. In P. J. Corr (Ed.), The reinforcement sensitivity theory of personality (pp. 120-154). Cambridge: Cambridge University Press. https://doi.org/10.1017/CBO9780511819384.005

Picó-Pérez, M., Radua, J., Steward, T., Menchón, J. M., \& Soriano-Mas, C. (2017). Emotion regulation in mood and anxiety disorders: A meta-analysis of fMRI cognitive reappraisal studies. Progress in Neuro-Psychopharmacology and Biological Psychiatry, 79(Pt B), 96104. https://doi.org/10.1016/j.pnpbp.2017.06.001

Pinto-Meza, A., Caseras, X., Soler, J., Puigdemont, D., Pérez, V., \& Torrubia, R. (2006). Behavioural Inhibition and Behavioural Activation Systems in current and recovered major depression participants. Personality and Individual Differences, 40(2), 215-226. https://doi.org/10.1016/j.paid.2005.06.021

Poy, R., Eixarch, M. del C., \& Ávila, C. (2004). On the relationship between attention and personality: Covert visual orienting of attention in anxiety and impulsivity. Personality and Individual Differences, 36(6), 1471-1481. https://doi.org/10.1016/S0191-8869(03)00242-3 
Ptak, R., Schnider, A., \& Fellrath, J. (2017). The Dorsal Frontoparietal Network: A Core System for Emulated Action. Trends in Cognitive Sciences, 21(8), 589-599. https://doi.org/10.1016/j.tics.2017.05.002

Qin, S., Young, C. B., Duan, X., Chen, T., Supekar, K., \& Menon, V. (2014). Amygdala Subregional Structure and Intrinsic Functional Connectivity Predicts Individual Differences in Anxiety During Early Childhood. Biological Psychiatry, 75(11), 892-900. https://doi.org/10.1016/J.BIOPSYCH.2013.10.006

Raichle, M. E. (2015). The restless brain: How intrinsic activity organizes brain function. Philosophical Transactions of the Royal Society B: Biological Sciences, 370(1668), 20140172. https://doi.org/10.1098/rstb.2014.0172

Rangel, A., \& Hare, T. (2010). Neural computations associated with goal-directed choice. Current Opinion in Neurobiology, 20(2), 262-270. https://doi.org/10.1016/j.conb.2010.03.001

Redgrave, P., Prescott, T. J., \& Gurney, K. (1999). Is the short-latency dopamine response too short to signal reward error? Trends in Neurosciences, 22(4), 146-151. https://doi.org/10.1016/S0166-2236(98)01373-3

Reinholdt-Dunne, M. L., Mogg, K., \& Bradley, B. P. (2009). Effects of anxiety and attention control on processing pictorial and linguistic emotional information. Behaviour Research and Therapy, 47(5), 410-417. https://doi.org/10.1016/j.brat.2009.01.012

Reuter, M, Stark, R., Hennig, J., Walter, B., Kirsch, P., Schienle, A., \& Vaitl, D. (2004). Personality and emotion: test of Gray's personality theory by means of an fMRI study. Behavioral Neuroscience, 118(3), 462-469. https://doi.org/10.1037/0735-7044.118.3.462

Reuter, Martin, Cooper, A. J., Smillie, L. D., Markett, S., \& Montag, C. (2015). A new measure for the revised reinforcement sensitivity theory: psychometric criteria and genetic $\begin{array}{lllll}\text { validation. Frontiers in Systems Neuroscience, } & 9, \quad 38 .\end{array}$ https://doi.org/10.3389/fnsys.2015.00038

Richter, A., \& Gruber, O. (2018). Influence of ventral tegmental area input on cortico-subcortical networks underlying action control and decision making. Human Brain Mapping, 39(2), 1004-1014. https://doi.org/10.1002/hbm.23899

Ridderinkhof, K. R., Ullsperger, M., Crone, E. A., \& Nieuwenhuis, S. (2004). The role of the medial frontal cortex in cognitive control. Science, 306(5695), 443-447. https://doi.org/10.1126/science.1100301

Ross, S. R., Benning, S. D., Patrick, C. J., Thompson, A., \& Thurston, A. (2009). Factors of the Psychopathic Personality Inventory. Assessment, 16(1), 71-87. 
https://doi.org/10.1177/1073191108322207

Ross, S. R., Moltó, J., Poy, R., Segarra, P., Pastor, M. C., \& Montañés, S. (2007). Gray’s model and psychopathy: BIS but not BAS differentiates primary from secondary psychopathy in noninstitutionalized young adults. Personality and Individual Differences, 43(7), 16441655. https://doi.org/10.1016/j.paid.2007.04.020

Rushworth, M. F. S., Behrens, T. E. J., Rudebeck, P. H., \& Walton, M. E. (2007). Contrasting roles for cingulate and orbitofrontal cortex in decisions and social behaviour. Trends in Cognitive Sciences, 11(4), 168-176. https://doi.org/10.1016/j.tics.2007.01.004

Rushworth, M. F. S., Noonan, M. P., Boorman, E. D., Walton, M. E., \& Behrens, T. E. (2011). Frontal Cortex and Reward-Guided Learning and Decision-Making. Neuron, 70(6), 10541069. https://doi.org/10.1016/J.NEURON.2011.05.014

Salavert, J., Caseras, X., Torrubia, R., Furest, S., Arranz, B., Dueñas, R., \& San, L. (2007). The functioning of the Behavioral Activation and Inhibition Systems in bipolar I euthymic patients and its influence in subsequent episodes over an eighteen-month period. Personality and Individual Differences, 42(7), 1323-1331. https://doi.org/10.1016/j.paid.2006.10.010

Salum, G. A., Mogg, K., Bradley, B. P., Gadelha, A., Pan, P., Tamanaha, A. C., ... Pine, D. S. (2013). Threat bias in attention orienting: Evidence of specificity in a large community$\begin{array}{llll}\text { based study. } \quad \text { Psychological } & \text { Medicine, 433-745), }\end{array}$ https://doi.org/10.1017/S0033291712001651

Schienle, A., Ebner, F., \& Schäfer, A. (2011). Localized gray matter volume abnormalities in generalized anxiety disorder. European Archives of Psychiatry and Clinical Neuroscience, 261(4), 303-307. https://doi.org/10.1007/s00406-010-0147-5

Schilling, C., Kühn, S., Paus, T., Romanowski, A., Banaschewski, T., Barbot, A., ... Gallinat, J. (2013). Cortical thickness of superior frontal cortex predicts impulsiveness and perceptual reasoning in adolescence. Molecular Psychiatry, 18(5), 624-630. https://doi.org/10.1038/mp.2012.56

Sesack, S. R., \& Grace, A. A. (2010). Cortico-Basal Ganglia Reward Network: Microcircuitry. Neuropsychopharmacology, 35(1), 27-47. https://doi.org/10.1038/npp.2009.93

Sescousse, G., Caldú, X., Segura, B., \& Dreher, J.-C. (2013). Processing of primary and secondary rewards: a quantitative meta-analysis and review of human functional neuroimaging studies. Neuroscience and Biobehavioral Reviews, 37(4), 681-696. https://doi.org/10.1016/j.neubiorev.2013.02.002

Shehzad, Z., Kelly, A. M. C., Reiss, P. T., Gee, D. G., Gotimer, K., Uddin, L. Q., .. Milham, M. 
P. (2009). The Resting Brain: Unconstrained yet Reliable. Cerebral Cortex, 19(10), 22092229. https://doi.org/10.1093/cercor/bhn256

Siegel, A., \& Sapru, H. N. (2010). Essential neuroscience (2nd ed.). Baltimore: Lippincott Williams \& Wilkins.

Silva, C., \& McNaughton, N. (2019). Are periaqueductal gray and dorsal raphe the foundation of appetitive and aversive control? A comprehensive review. Progress in Neurobiology, 177, 33-72. https://doi.org/10.1016/j.pneurobio.2019.02.001

Simon, J. J., Walther, S., Fiebach, C. J., Friederich, H.-C., Stippich, C., Weisbrod, M., \& Kaiser, S. (2010). Neural reward processing is modulated by approach- and avoidance-related $\begin{array}{llll}\text { personality } & \text { traits. } & \text { NeuroImage, } & 49(2),\end{array}$ https://doi.org/10.1016/J.NEUROIMAGE.2009.09.016

Simons, J. S., Dvorak, R. D., \& Batien, B. D. (2008). Methamphetamine Use in a Rural College Population: Associations With Marijuana Use, Sensitivity to Punishment, and Sensitivity to Reward. Psychology of Addictive Behaviors, 22(3), 444-449. https://doi.org/10.1037/0893164X.22.3.444

Slobodskaya, H. R. (2016). The contribution of reinforcement sensitivity to the personalitypsychopathology hierarchical structure in childhood and adolescence. Journal of Abnormal Psychology, 125(8), 1067-1078. https://doi.org/10.1037/abn0000179

Smederevac, S., Mitrović, D., Čolović, P., \& Nikolašević, Ž. (2014). Validation of the measure of revised reinforcement sensitivity theory constructs. Journal of Individual Differences, 35(1), 12-21. https://doi.org/10.1027/1614-0001/a000121

Smillie, L. D. (2008). What is reinforcement sensitivity? Neuroscience paradigms for approachavoidance process theories of personality. European Journal of Personality, 22(5), 359384. https://doi.org/10.1002/per.674

Smillie, L. D., Pickering, A. D., \& Jackson, C. J. (2006). The New Reinforcement Sensitivity Theory: Implications for Personality Measurement. Personality and Social Psychology Review, 10(4), 320-335. https://doi.org/10.1207/s15327957pspr1004_3

Smith, S. M., Fox, P. T., Miller, K. L., Glahn, D. C., Fox, P. M., Mackay, C. E., ... Beckmann, C. F. (2009). Correspondence of the brain's functional architecture during activation and rest. Proceedings of the National Academy of Sciences of the United States of America, 106(31), 13040-13045. https://doi.org/10.1073/pnas.0905267106

Smoller, J. W., Gallagher, P. J., Duncan, L. E., McGrath, L. M., Haddad, S. A., Holmes, A. J., ... Cohen, B. M. (2014). The human ortholog of acid-sensing ion channel gene ASIC1a is 
associated with panic disorder and amygdala structure and function. Biological Psychiatry, 76(11), 902-910. https://doi.org/10.1016/j.biopsych.2013.12.018

Spielberger, C. D., Gorsuch, R. L., \& Lushene, R. (1970). Manual for the State Trait Anxiety Inventory. Palo Alto, CA: Consulting Psychologist Press.

Sportel, B. E., Nauta, M. H., de Hullu, E., de Jong, P. J., \& Hartman, C. A. (2011). Behavioral Inhibition and Attentional Control in Adolescents: Robust Relationships with Anxiety and Depression. Journal of Child and Family Studies, 20(2), 149-156. https://doi.org/10.1007/s10826-010-9435-y

Stam, D., Huang, Y.-A., \& Van den Stock, J. (2019). Non-overlapping and Inverse Associations Between the Sexes in Structural Brain-Trait Associations. Frontiers in Psychology, 10, 904. https://doi.org/10.3389/fpsyg.2019.00904

Struijs, S. Y., Lamers, F., Rinck, M., Roelofs, K., Spinhoven, P., \& Penninx, B. W. J. H. (2018). The predictive value of Approach and Avoidance tendencies on the onset and course of depression and anxiety disorders. Depression and Anxiety, 35(6), 551-559. https://doi.org/10.1002/da.22760

Sylvers, P., Lilienfeld, S. O., \& LaPrairie, J. L. (2011). Differences between trait fear and trait anxiety: Implications for psychopathology. Clinical Psychology Review, 31(1), 122-137. https://doi.org/10.1016/j.cpr.2010.08.004

Taylor, J., Reeves, M., James, L., \& Bobadilla, L. (2006). Disinhibitory trait profile and its relation to Cluster B personality disorder features and substance use problems. European Journal of Personality, 20(4), 271-284. https://doi.org/10.1002/per.585

Tomasino, B., \& Gremese, M. (2016). Effects of Stimulus Type and Strategy on Mental Rotation Network: An Activation Likelihood Estimation Meta-Analysis. Frontiers in Human Neuroscience, 9, 693. https://doi.org/10.3389/fnhum.2015.00693

Torrubia, R., Avila, C., \& Caseras, X. (2008). Reinforcement sensitivity scales. In P. J. Corr (Ed.), The reinforcement sensitivity theory of personality (pp. 188-226). New York: Cambridge University Press.

Torrubia, R., Ávila, C., Moltó, J., \& Caseras, X. (2001). The Sensitivity to Punishment and Sensitivity to Reward Questionnaire (SPSRQ) as a measure of Gray's anxiety and impulsivity dimensions. Personality and Individual Differences, 31(6), 837-862. https://doi.org/10.1016/S0191-8869(00)00183-5

Torrubia, R., \& Tobeña, A. (1984). A scale for the assessment of "susceptibility to punishment" as a measure of anxiety: preliminary results. Personality and Individual Differences, 5(3), 
371-375. https://doi.org/10.1016/0191-8869(84)90078-3

Tovote, P., Fadok, J. P., \& Lüthi, A. (2015). Neuronal circuits for fear and anxiety. Nature Reviews. Neuroscience, 16(6), 317-331. https://doi.org/10.1038/nrn3945

Tzschoppe, J., Nees, F., Banaschewski, T., Barker, G. J., Büchel, C., Conrod, P. J., ... Flor, H. (2014). Aversive learning in adolescents: Modulation by amygdala-prefrontal and amygdala-hippocampal connectivity and neuroticism. Neuropsychopharmacology, 39(4), 875-884. https://doi.org/10.1038/npp.2013.287

Urošević, S., Collins, P., Muetzel, R., Schissel, A., Lim, K. O., \& Luciana, M. (2015). Effects of reward sensitivity and regional brain volumes on substance use initiation in adolescence. Social Cognitive and Affective Neuroscience, 10(1), 106-113. https://doi.org/10.1093/scan/nsu022

Uzieblo, K., Verschuere, B., \& Crombez, G. (2007). The Psychopathic Personality Inventory: Construct validity of the two-factor structure. Personality and Individual Differences, 43(4), 657-667. https://doi.org/10.1016/j.paid.2007.01.008

van den Heuvel, M. P., \& Hulshoff Pol, H. E. (2010). Exploring the brain network: A review on resting-state fMRI functional connectivity. European Neuropsychopharmacology, 20(8), 519-534. https://doi.org/10.1016/j.euroneuro.2010.03.008

Van Schuerbeek, P., Baeken, C., De Raedt, R., De Mey, J., \& Luypaert, R. (2011). Individual differences in local gray and white matter volumes reflect differences in temperament and character: A voxel-based morphometry study in healthy young females. Brain Research, 1371, 32-42. https://doi.org/10.1016/j.brainres.2010.11.073

Vassena, E., Krebs, R. M., Silvetti, M., Fias, W., \& Verguts, T. (2014). Dissociating contributions of ACC and vmPFC in reward prediction, outcome, and choice. Neuropsychologia, 59, 112123. https://doi.org/10.1016/J.NEUROPSYCHOLOGIA.2014.04.019

Vervoort, L., Wolters, L. H., Hogendoorn, S. M., de Haan, E., Boer, F., \& Prins, P. J. M. (2010). Sensitivity of Gray's Behavioral Inhibition System in clinically anxious and non-anxious children and adolescents. Personality and Individual Differences, 48(5), 629-633. https://doi.org/10.1016/j.paid.2009.12.021

Vollema, M. G., \& van den Bosch, R. J. (1995). The Multidimensionality of Schizotypy. Schizophrenia Bulletin, 21(1), 19-31. https://doi.org/10.1093/schbul/21.1.19

Wang, S., Kong, F., Zhou, M., Chen, T., Yang, X., Chen, G., \& Gong, Q. (2017). Brain Structure Linking Delay Discounting and Academic Performance. Human Brain Mapping, 38(8), 3917-3926. https://doi.org/10.1002/hbm.23638 
Wiesmann, M., Kopietz, R., Albrecht, J., Linn, J., Reime, U., Kara, E., ... Stephan, T. (2006). Eye closure in darkness animates olfactory and gustatory cortical areas. NeuroImage, 32(1), 293-300. https://doi.org/10.1016/j.neuroimage.2006.03.022

Wilson, G. D., Barrett, P. T., \& Gray, J. A. (1989). Human reactions to reward and punishment: A questionnaire examination of Gray's personality theory. British Journal of Psychology, 80(4), 509-515. https://doi.org/10.1111/j.2044-8295.1989.tb02339.x

Winkler, A. M., Greve, D. N., Bjuland, K. J., Nichols, T. E., Sabuncu, M. R., Håberg, A. K., ... Rimol, L. M. (2018). Joint Analysis of Cortical Area and Thickness as a Replacement for the Analysis of the Volume of the Cerebral Cortex. Cerebral Cortex, 28(2), 738-749. https://doi.org/10.1093/cercor/bhx308

Winkler, A. M., Kochunov, P., Blangero, J., Almasy, L., Zilles, K., Fox, P. T., ... Glahn, D. C. (2010). Cortical thickness or grey matter volume? The importance of selecting the phenotype for imaging genetics studies. NeuroImage, 53(3), 1135-1146. https://doi.org/10.1016/j.neuroimage.2009.12.028

Wise, R. A. (2004). Dopamine, learning and motivation. Nature Reviews. Neuroscience, 5(6), 483-494. https://doi.org/10.1038/nrn1406

Wise, R. A. (2009). Roles for nigrostriatal-not just mesocorticolimbic-dopamine in reward and addiction. Trends in Neurosciences, 32(10), 517-524. https://doi.org/10.1016/j.tins.2009.06.004

Wright, C. I., Williams, D., Feczko, E., Barrett, L. F., Dickerson, B. C., Schwartz, C. E., \& Wedig, M. M. (2006). Neuroanatomical Correlates of Extraversion and Neuroticism. Cerebral Cortex, 16(12), 1809-1819. https://doi.org/10.1093/cercor/bhj118

Yen, J.-Y., Cheng-Fang, Y., Chen, C.-S., Chang, Y.-H., Yeh, Y.-C., \& Ko, C.-H. (2012). The bidirectional interactions between addiction, behaviour approach and behaviour inhibition systems among adolescents in a prospective study. Psychiatry Research, 200(2-3), 588592. https://doi.org/10.1016/J.PSYCHRES.2012.03.015

Yeo, B. T. T., Krienen, F. M., Sepulcre, J., Sabuncu, M. R., Lashkari, D., Hollinshead, M., ... Buckner, R. L. (2011). The organization of the human cerebral cortex estimated by intrinsic functional connectivity. Journal of Neurophysiology, 106(3), 1125-1165. https://doi.org/10.1152/jn.00338.2011

Yiend, J., \& Mathews, A. (2001). Anxiety and attention to threatening pictures. Quarterly Journal of Experimental Psychology Section A: Human Experimental Psychology, 54(3), 665-681. https://doi.org/10.1080/713755991 


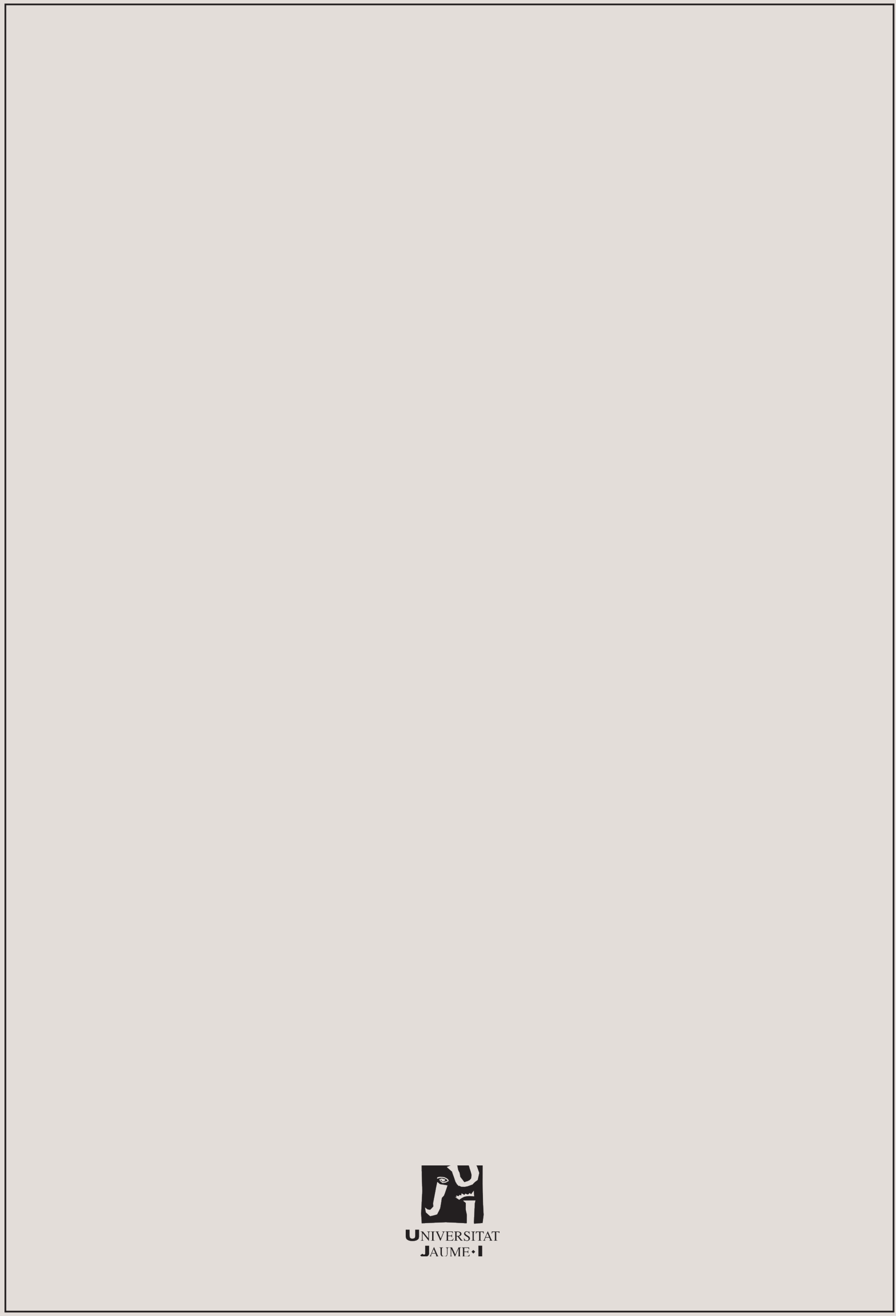

